H. 2947 TREE-1365

Distribution Category:

UC-66d

\title{
MASTER
}

\section{ANALYSIS OF BINARY THERMODYNAMIC CYCLES FOR A MODERATELY LOW-TEMPERATURE GEOTHERMAL RESOURCE}

\author{
O. Jack Demuth
}

JULY 1979

EGгG Idaho, Inc. 


\section{DISCLAIMER}

This report was prepared as an account of work sponsored by an agency of the United States Government. Neither the United States Government nor any agency Thereof, nor any of their employees, makes any warranty, express or implied, or assumes any legal liability or responsibility for the accuracy, completeness, or usefulness of any information, apparatus, product, or process disclosed, or represents that its use would not infringe privately owned rights. Reference herein to any specific commercial product, process, or service by trade name, trademark, manufacturer, or otherwise does not necessarily constitute or imply its endorsement, recommendation, or favoring by the United States Government or any agency thereof. The views and opinions of authors expressed herein do not necessarily state or reflect those of the United States Government or any agency thereof. 


\section{DISCLAIMER}

Portions of this document may be illegible in electronic image products. Images are produced from the best available original document. 
Printed in the United States of America Available from

National Technical Information Service

U.S. Department of Commerce

5285 Port Royal Road

Springfield, Virginia 22161

Price: Printed Copy $\$ 6.50$; Microfiche $\$ 3.00$

This report was prepared as an account of work sponsored by the United States Government. Neither the United States nor the Department of Energy, nor any of their employees, nor any of their contractors, subcontractors, or their employees, makes any warranty, express or implied, or assumes any legal liability or responsibility for the accuracy, completeness or usefulness of any information, apparatus, product or process disclosed, or represents that its use would not infringe privately owned rights. 


\title{
ANALYSIS OF BINARY THERMODYNAMIC CYCLES FOR A MODERATELY LOW-TEMPERATURE GEOTHERMAL RESOURCE
}

\author{
O. Jack Demuth
}

\section{EG\&G IDAHO, INC.}

Published July 1979

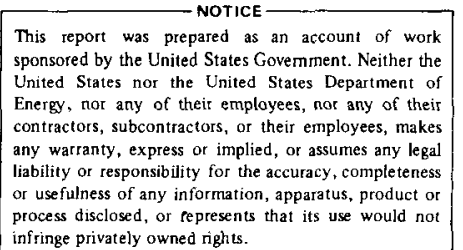

PREPARED FOR THE U.S. DEPARTMENT OF ENERGY

IDAHO OPERATIONS OFFICE

UNDER CONTRACT NO. DE-AC07-76IDO1570 


\begin{abstract}
Analyses of a number of geothermal binary-cycles were made with the objective of finding a cycle which can produce low-cost electrical energy from a moderately low-temperature geothermal resource.

Cycles were screened which included isobutane, pentane, cis-2-butene, and several mixed-hydrocarbon working fluids. Dual- and triple-boiling cycles were analyzed. Both shell-and-tube and direct-contact boilers, heaters, and condensers were assessed. A geothermal fluid (geo-fluid), typical of Raft River resource conditions was assumed, which has a temperature of $290^{\circ} \mathrm{F}$ and 52 parts per million dissolved nitrogen. Special emphasis in the analyses was directed toward investigation of several methods for keeping the loss of working fluid for the cycle at an acceptable level.

It was concluded that for the Raft River geo-fluid, the direct-contact cycle has a potential for net geofluid utilization effectiveness values, (watt-hr/lbm geo-fluid) equivalent to those of the shell-and-tube cycle. Therefore, because of the lower cost of direct-contact components, a potential exists for the directcontact plant to produce lower cost electrical energy than a comparable shell-and-tube plant. Advanced cycles were assessed which showed improvements in net geo-fluid utilization effectiveness, relative to the first Raft River 5-MW Pilot Plant (dual-boiling, shell-and-tube isobutane cycle), of up to $19 \%$.
\end{abstract}




\section{PREFACE}

English units are used throughout the report. The thermodynamic properties used in the calculations were available in English units, and those units are most familiar to the practicing U.S. engineers in the field of geothermal-electrical power. For convenience of converting the various groupings found in the report to SI units, the following table is provided.

\section{To Convert From}

Btu

$\overline{\mathrm{lbm}}$

Btu

$\overline{\mathrm{lbm}{ }^{\circ} \mathrm{F}}$

$\frac{\mathrm{ft}^{3}}{\mathrm{lbm}}$

$\frac{\mathrm{lbm}}{\mathrm{hr}}$

$\mathrm{hr}$

psi

$\underline{\text { watt-hr }}$

$\mathrm{lbm}$

Temperature

change ${ }^{\mathrm{O}} \mathrm{F}$
To

Multiply By

2326

$\overline{\text { kilogram }}$

joule

$\overline{\text { kilogram kelvin }}$

$\frac{\text { metre }^{3}}{\text { kilogram }}$

kilogram

$\mathrm{hr}$

pascal

watt-hr

kilogram

Temperature

change ${ }^{\circ} \mathrm{C}$
6895

2.205

4187

0.4536

$5 / 9$

$\left(32^{\circ} \mathrm{F}=0^{\circ} \mathrm{C}\right)$ 


\begin{tabular}{|c|c|c|}
\hline ACT & $=$ & actual \\
\hline BOIL & $=$ & boiler \\
\hline $\mathrm{C} 3$ & $=$ & propane \\
\hline $\mathrm{C} 4$ & $=$ & butane \\
\hline $\mathrm{C} 5$ & $=$ & pentane \\
\hline C6 & $=$ & hexane \\
\hline $\mathrm{C}_{\mathrm{PGF}}$ & $=$ & specific heat of geo-fluid \\
\hline $\mathrm{C}_{\mathrm{PS}}$ & $=$ & specific heat of liquid solute \\
\hline $\mathrm{CON}$ & $=$ & condenser \\
\hline $\mathrm{CW}$ & $=$ & cooling water \\
\hline $\mathrm{DC}$ & $=$ & direct contact \\
\hline EX & $=$ & exhaust \\
\hline FL & $=$ & flash \\
\hline GF & $=$ & geo-fluid \\
\hline$H^{\prime}$ & $=$ & modified Henry constant \\
\hline$h$ & $=$ & specific enthalpy \\
\hline$h_{f g}$ & $=$ & heat of vaporization \\
\hline $\mathrm{HP}$ & $=$ & high pressure \\
\hline$\Delta \mathrm{h}_{\mathrm{SOLN}}$ & $=$ & heat of solution \\
\hline IC4 & $=$ & isobutane \\
\hline IC5 & $=$ & isopentane \\
\hline in & $=$ & entering a component \\
\hline IS & $=$ & isentropic \\
\hline LP & $=$ & low pressure \\
\hline M & $=$ & molecular weight \\
\hline $\mathrm{MP}$ & $=$ & medium pressure \\
\hline $\mathrm{N}_{2}$ & $=$ & nitrogen \\
\hline $\mathrm{NC}$ & $=$ & noncondensibles \\
\hline $\mathrm{O}_{2}$ & $=$ & oxygen \\
\hline out & $=$ & leaving a component \\
\hline $\mathrm{P}$ & $=$ & pressure \\
\hline
\end{tabular}




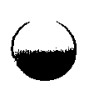

\begin{tabular}{|c|c|c|}
\hline $\mathrm{P}_{\mathrm{C}}$ & $=$ & condenser pressure \\
\hline $\mathrm{P}_{\mathrm{NC}}$ & $=$ & noncondensibles pressure \\
\hline Q & $=$ & heat exchanged \\
\hline S & $=$ & solute dissolved in geo-fluid \\
\hline SL & $=$ & saturated liquid \\
\hline STM & $=$ & water vapor \\
\hline SV & $=$ & saturated vapor \\
\hline $\mathrm{T}$ & $=$ & turbine \\
\hline $\mathrm{T}_{\mathrm{C}}$ & $=$ & condensing temperature \\
\hline$\Delta T_{P B}$ & $=$ & boiler pinch point \\
\hline$\Delta \mathrm{T}_{\mathrm{PC}}$ & $=$ & condenser pinch point \\
\hline W & $=$ & weight flow \\
\hline WF & $=$ & working fluid \\
\hline WFE & $=$ & working fluid evaporated \\
\hline WFL & $=$ & working-fluid liquid \\
\hline WFV & $=$ & working-fluid vapor \\
\hline WL & $=$ & water liquid \\
\hline$\eta_{\mathrm{P}}$ & $=$ & pump efficiency \\
\hline$\gamma$ & $=$ & ratio of specific heats \\
\hline
\end{tabular}




\section{CONTENTS}

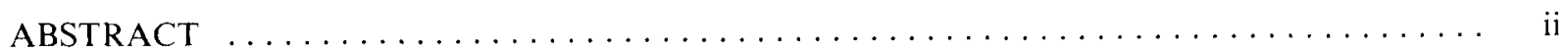

PREFACE $\ldots \ldots \ldots \ldots \ldots \ldots \ldots \ldots \ldots \ldots \ldots \ldots \ldots \ldots \ldots \ldots \ldots \ldots \ldots \ldots \ldots \ldots \ldots \ldots \ldots$

NOMENCLATURE $\ldots \ldots \ldots \ldots \ldots \ldots \ldots \ldots \ldots \ldots \ldots \ldots \ldots \ldots \ldots \ldots \ldots \ldots \ldots \ldots \ldots$

1. INTRODUCTION AND SUMMARY $\ldots \ldots \ldots \ldots \ldots \ldots \ldots \ldots \ldots \ldots \ldots \ldots \ldots \ldots$

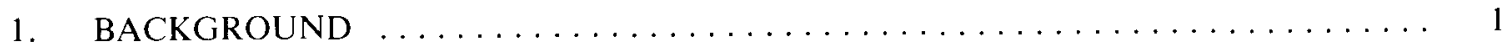

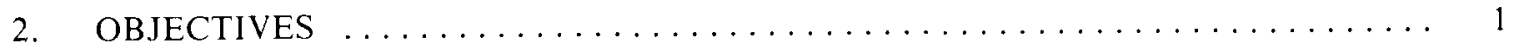

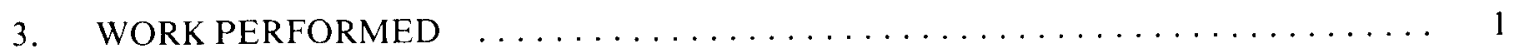

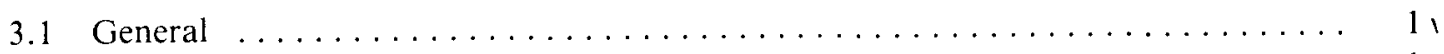

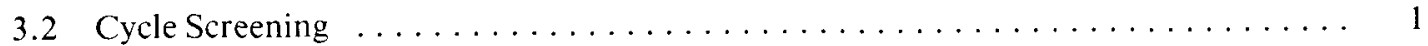

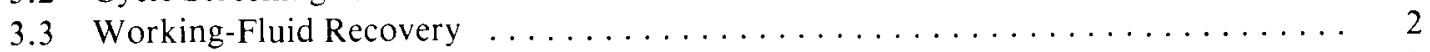

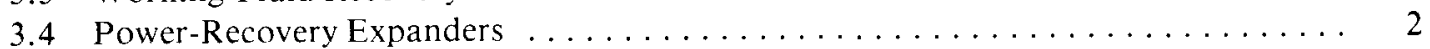

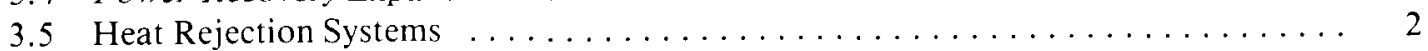

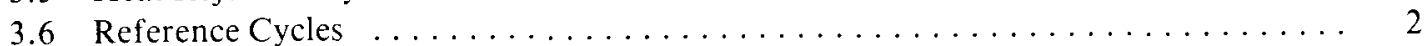

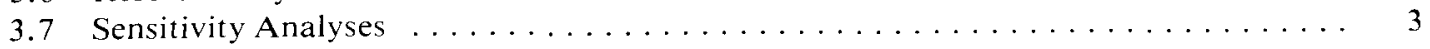

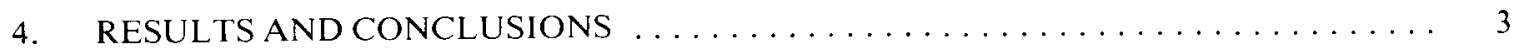

II. DESCRIPTION OF THE CYCLES $\ldots \ldots \ldots \ldots \ldots \ldots \ldots \ldots \ldots \ldots \ldots \ldots \ldots \ldots \ldots$

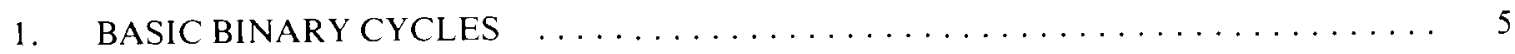

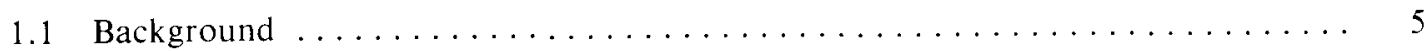

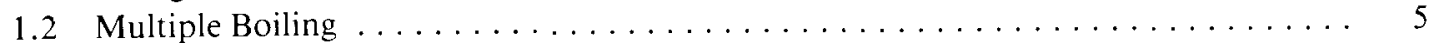

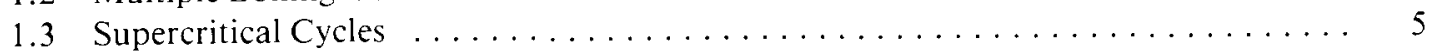

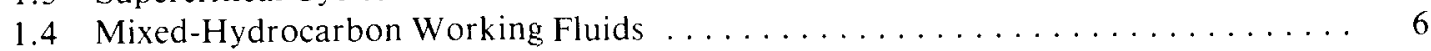

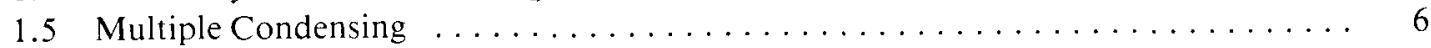

2. DIRECT-CONTACT CYCLES $\ldots \ldots \ldots \ldots \ldots \ldots \ldots \ldots \ldots \ldots \ldots \ldots \ldots \ldots \ldots \ldots \ldots \ldots \ldots \ldots \ldots \ldots$

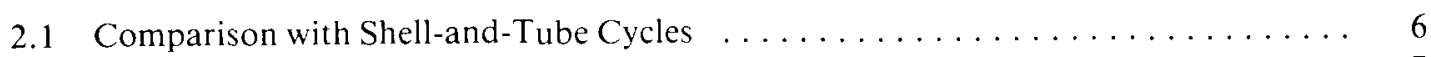

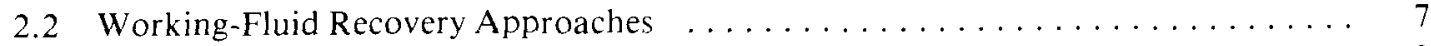

2.3 Optional Power-Recovery Expanders $\ldots \ldots \ldots \ldots \ldots \ldots \ldots \ldots \ldots \ldots$

III. ANALYSIS METHODS USED FOR DIRECT-CONTACT, STATE POINT,

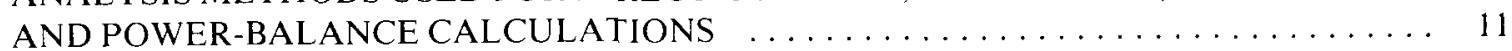

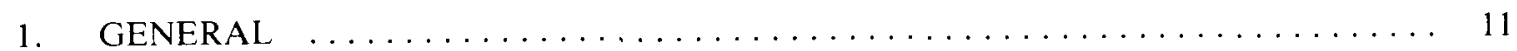

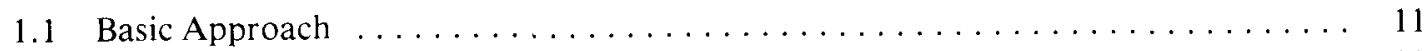

1.2 Alternate Methods for Treating Vapor Mixtures $\ldots \ldots \ldots \ldots \ldots \ldots \ldots \ldots \ldots \ldots \ldots$

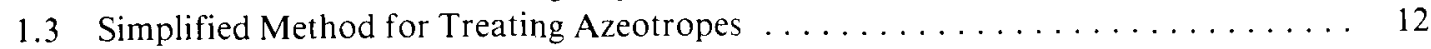

2. BOILER STATE POINT CALCULATION METHOD $\ldots \ldots \ldots \ldots \ldots \ldots \ldots \ldots \ldots$ 


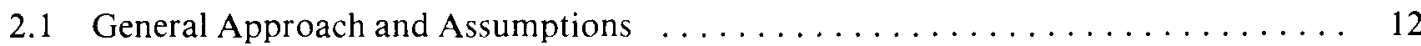

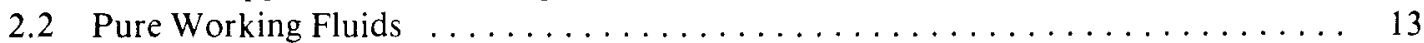

2.3 Mixed-Hydrocarbon Working Fluids $\ldots \ldots \ldots \ldots \ldots \ldots \ldots \ldots \ldots \ldots$

3. HEATER CALCULATIONAL METHOD $\ldots \ldots \ldots \ldots \ldots \ldots \ldots \ldots \ldots \ldots \ldots \ldots \ldots$

4. CALCULATIONAL METHODS FOR TURBINES, LIQUID PUMPS, AND VAPOR COMPRESSORS $\ldots \ldots \ldots \ldots \ldots \ldots \ldots \ldots \ldots \ldots \ldots \ldots \ldots \ldots \ldots \ldots \ldots$

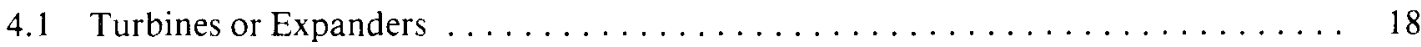

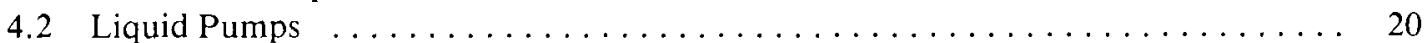

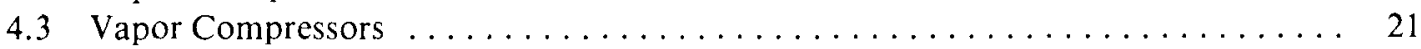

5. DIRECT-CONTACT CONDENSER CALCULATIONAL METHOD $\ldots \ldots \ldots \ldots \ldots 22$

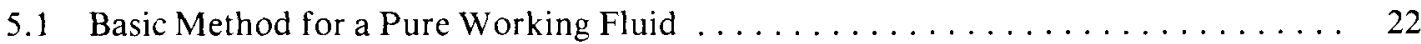

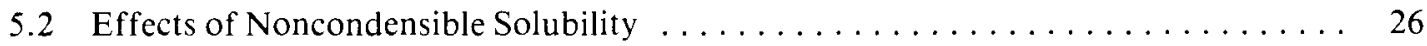

5.3 Condensing of Mixed-Hydrocarbon Working Fluids $\ldots \ldots \ldots \ldots \ldots \ldots \ldots$

6. STEADY-FLOW EQUILIBRIUM FLASHING $\ldots \ldots \ldots \ldots \ldots \ldots \ldots \ldots \ldots \ldots \ldots \ldots \ldots$

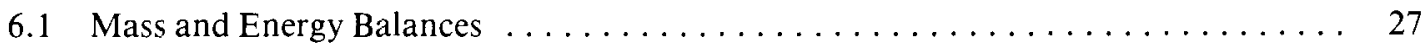

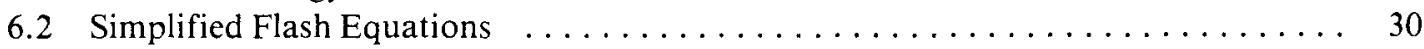

6.3 Flashing with Several Dissolved Gases $\ldots \ldots \ldots \ldots \ldots \ldots \ldots \ldots \ldots \ldots \ldots \ldots$

7. REFRIGERATION CALCULATIONS $\ldots \ldots \ldots \ldots \ldots \ldots \ldots \ldots \ldots \ldots \ldots \ldots \ldots$

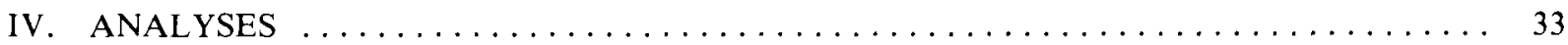

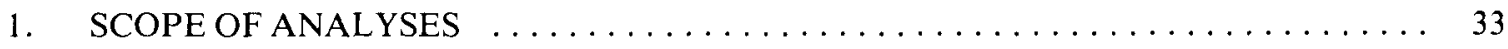

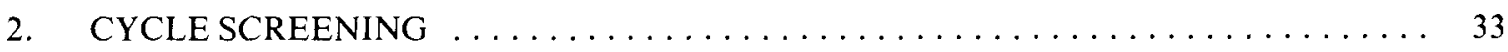

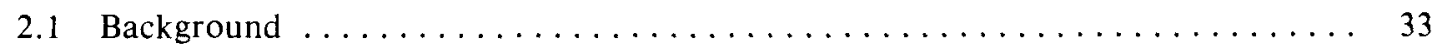

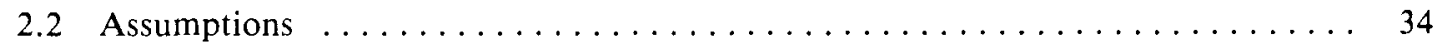

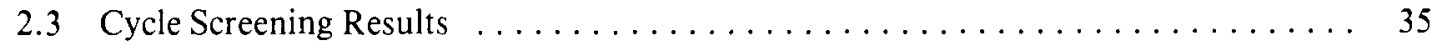

3. WORKING-FLUID RECOVERY $\ldots \ldots \ldots \ldots \ldots \ldots \ldots \ldots \ldots \ldots \ldots \ldots \ldots \ldots \ldots \ldots$

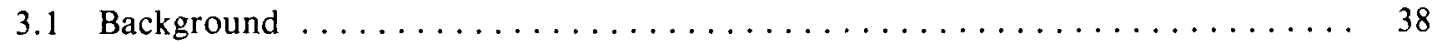

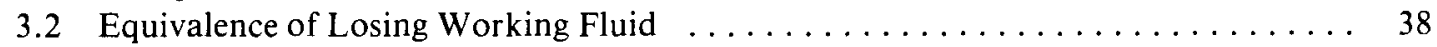

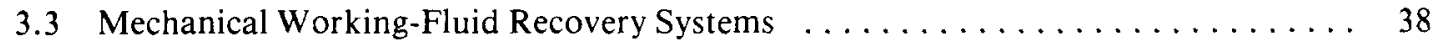

3.4 Chemical Working-Fluid Recovery Systems $\ldots \ldots \ldots \ldots \ldots \ldots \ldots \ldots \ldots \ldots \ldots$

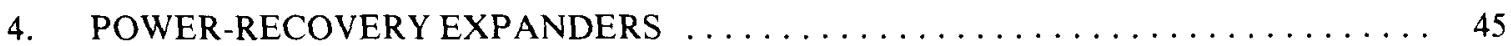

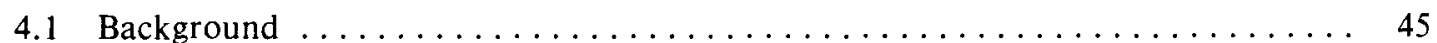

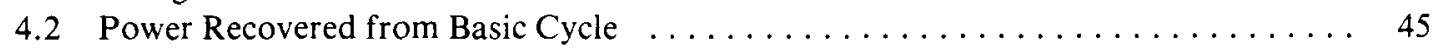

4.3 Expanders in the Working-Fluid Recovery System $\ldots \ldots \ldots \ldots \ldots \ldots \ldots \ldots$

5. PRELIMINARY ASSESSMENT OF HEAT-REJECTION SYSTEMS $\ldots \ldots \ldots \ldots \ldots$

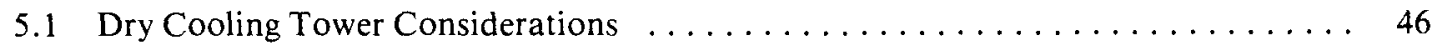

5.2 Comparison of Several Heat-Rejection Systems $\ldots \ldots \ldots \ldots \ldots \ldots \ldots \ldots \ldots$ 


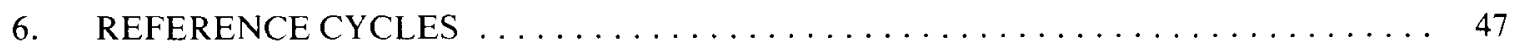

6.1 General $\ldots \ldots \ldots \ldots \ldots \ldots \ldots \ldots \ldots \ldots \ldots \ldots \ldots \ldots \ldots \ldots$

6.2 Dual-Boiling, Direct-Contact Pentane Cycle $\ldots \ldots \ldots \ldots \ldots \ldots \ldots \ldots \ldots$

6.3 Dual-Boiling, Direct-Contact Isobutane Cycle . . . . . . . . . . . . . . . . . . . 49

6.4 Triple-Boiling, Triple-Condensing, Direct-

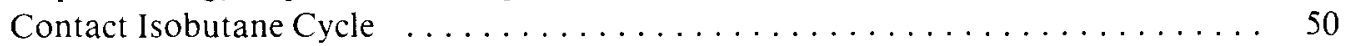

6.5 Triple-Boiling, Triple-Condensing, Shell-and-

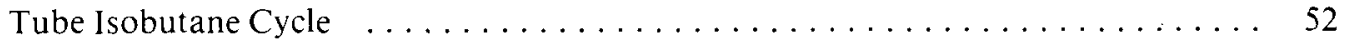

V. OVERALL CONCLUSIONS AND RECOMMENDATIONS $\ldots \ldots \ldots \ldots 4$

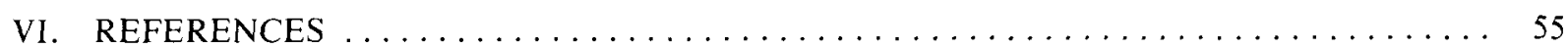

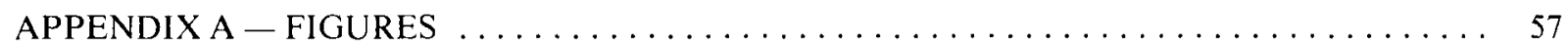

APPENDIX B - TABLES $\ldots \ldots \ldots \ldots \ldots \ldots \ldots \ldots$

\section{FIGURES}

1. Dual-boiling, direct-contact geothermal cycle; basic cycle components . . . . . . . . . . 59

2. Temperature - heat exchanged diagram for dual-boiling isobutane cycle $\ldots \ldots \ldots$

3. Temperature - heat exchanged diagram for triple-boiling, triple-condensing isobutane cycle ... 61

4. Temperature - heat exchanged diagram for dual-boiling 0.5 IC $4 / 0.5$ IC6 cycle $\ldots \ldots \ldots 2$

5. Triple-boiling, triple-condensing, direct-contact geothermal cycle with

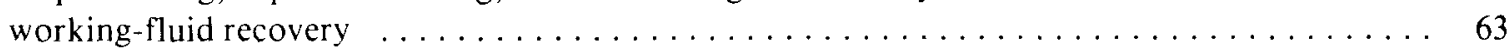

6. Dual-boiling, direct-contact cycle with working-fluid recovery $\ldots \ldots \ldots$

7. Effect of $\mathrm{N}_{2}$ partial pressure in condenser on cycle performance; condenser-vent products compressed and cooled $\ldots \ldots \ldots \ldots \ldots \ldots \ldots \ldots$

8. Effect of $\mathrm{N}_{2}$ partial pressure in condenser on cycle performance; condenser-vent products refrigerated $\ldots \ldots \ldots \ldots \ldots \ldots \ldots \ldots \ldots \ldots \ldots$

9. Effect of preflashing geo-fluid upstream of boilers for direct-contact, dual-boiling pentane cycle

10. Effect of preflash with heat-recovery evaporator on dual-boiling, direct-contact cycle $\ldots \ldots 6$

11. Power required for recovery of dissolved pentane by geo-fluid flashing process $\ldots \ldots \ldots \ldots$

\section{TABLES}

I. Comparison of Geothermal Cycles Using Raft River Geo-Fluid (Direct-Contact Cycles) . . . . 73

II. Comparison of Geothermal Cycles Using Raft River Geo-Fluid (Shell-and-Tube Cycles) . . . 74 
III. Working-Fluid Recovery Parasitics for Triple-Boiling, Isobutane, Direct-Contact Cycle with Shell-and-Tube Condenser (Raft River Conditions)

IV. Power-Recovery Expanders for Direct-Contact Cycles $\ldots \ldots \ldots \ldots \ldots \ldots \ldots \ldots \ldots \ldots$

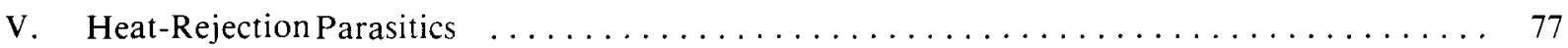

VI. State Points for Direct-Contact, Dual-Boiling Pentane Cycle $\ldots \ldots \ldots \ldots \ldots \ldots \ldots \ldots .79$

VII. Power Balance for Direct-Contact, Dual-Boiling Pentane Cycle $\ldots \ldots \ldots \ldots \ldots \ldots \ldots \ldots$

VIII. State Points for Direct-Contact, Dual-Boiling Isobutane Cycle $\ldots \ldots \ldots \ldots \ldots \ldots \ldots \ldots$

IX. Power Balance for Direct-Contact, Dual-Boiling Isobutane Cycle $\ldots \ldots \ldots \ldots \ldots \ldots \ldots . \quad 87$

X. State Points for Triple-Boiling, Triple-Condensing Direct-Contact, Isobutane Cycle $\ldots \ldots \ldots$

XI. Power Balance for Triple-Boiling, Triple-Condensing, Direct-Contact Isobutane Cycle $\ldots \ldots .93$

XII. State Points for Triple-Boiling, Triple-Condensing Shell-and-Tube Isobutane Cycle $\ldots \ldots \ldots 94$

XIII. Power Balance for Triple-Boiling, Triple-Condensing, Shell-and-Tube Isobutane Cycle $\ldots \ldots .97$

XIV. Effect of Boiler Temperatures on Cycle Performance for an Isobutance

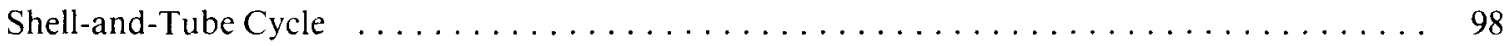




\section{ANALYSIS OF BINARY THERMODYNAMIC CYCLES FOR A MODERATELY LOW-TEMPERATURE GEOTHERMAL RESOURCE}

\section{INTRODUCTION AND SUMMARY}

\section{BACKGROUND}

The analyses discussed in this report were directed toward the design of a second Raft River 5-megawatt (MW) Geothermal Pilot Plant. The study is part of the Utilization Technology effort being conducted by EG\&G Idaho, Inc., for the Department of Energy, Division of Geothermal Energy, under Contract No. DE-AC07-76IDO1570.

The dual-boiling binary cycle was selected for the first 5-MW Raft River Pilot Plant to utilize the lower temperature resources (near $300^{\circ} \mathrm{F}$ ), and to develop the full potential of the high-temperature resources through the use of a combined cycle using a binary as a bottoming cycle in connection with the flash steam cycle. The current objective of the Utilization Technology effort on the second Raft River 5-MW Pilot Plant is to develop the concept of an advanced binary cycle which will produce low-cost electrical energy using the Raft River geothermal resource. In particular, use of direct-contact heat exchangers is being examined for the second plant because of their potential for resulting in lower component costs and possibly superior component thermodynamic performance. The preconceptual design work, initiated in FY 1978 and planned for completion in FY 1979, will lead to an optimized cycle with selected working fluid and state points.

\section{OBJECTIVES}

The specific objectives of the effort reported here are: (a) to evaluate the general behavior of multipleboiling, direct-contact binary cycles relative to comparable shell-and-tube cycles, and investigate methods for reducing working-fluid loss to an acceptable level, (b) to present a practicable direct-contact, binary cycle configuration that will show a marked improvement in performance relative to the first 5-MW Pilot Plant, and assess its power balance and working-fluid losses, and (c) to present an improved shell-and-tube cycle which will show a comparable improvement in performance relative to the first 5-MW Pilot Plant.

\section{WORK PERFORMED}

\subsection{General}

The work performed consists of assembling analysis methods required to determine the state points and power balances of direct-contact and shell-and-tube cycles, and then of analyzing a number of cycles using these methods. For the direct-contact cycles emphasis was placed on evaluating the power penalties associated with recovery of an acceptable fraction of the working fluid which would be either dissolved in the reinjected geo-fluid, vented to the atmosphere with noncondensibles from the condenser, or released to the atmosphere from the cooling water in a wet cooling tower.

\subsection{Cycle Screening}

For the present study several categories of analyses were conducted. In the first category, a number of cycles were screened which included isobutane, pentane, cis-2-butene, and several mixed-hydrocarbon working fluids. Dual- and triple-boiling cycles were analyzed with both single and staged condensing. Both shell-and-tube and direct-contact boilers, heaters, and condensers were assessed. Wet cooling towers were 
assumed. For the cycle-screening effort working-fluid recovery parasitic losses were not assessed. Raft River geo-fluid, with a temperature of $290^{\circ} \mathrm{F}$ and 52 parts per million dissolved nitrogen, was assumed for all analyses.

\subsection{Working-Fluid Recovery}

A second category of effort consists of the assessment of parasitic losses associated with recovery of working fluid for the direct-contact cycles. Analyses were conducted for pentane and isobutane cycles which utilized geo-fluid at Raft River conditions.

Power requirements for recovering working fluid were assessed parametrically, as a function of fluid loss, for several mechanical recovery approaches. To recover working fluid from the condenser vent, the approaches evaluated were: (a) refrigeration of the condenser vent products, (b) compressing and cooling the vent products, and (c) preflashing the geo-fluid upstream of the boiler. Postflashing the geo-fluid before reinjection was the approach examined for recovery of working fluid dissolved in the geo-fluid. Flashing of the cooling water was examined for recovery of working fluid dissolved in cooling water.

Brief assessments of several chemical working-fluid recovery methods were made by Energy Inc. of Idaho Falls, Idaho and by E. Hoffman energy consultant to the Idaho National Engineering Laboratory (INEL).

Some consideration was given to the magnitudes of working-fluid loss which might be considered acceptable. These considerations suggested that for a 5-MW plant, the loss should be kept as low as $30 \mathrm{lbm} / \mathrm{hr}$. The corresponding loss for a $50-\mathrm{MW}$ plant would be about $300 \mathrm{lbm} / \mathrm{hr}$, and would represent an increase in the cost of electrical energy of about $1 \%$.

\subsection{Power-Recovery Expanders}

A third category of effort includes evaluation of optional power-recovery expanders. These expanders can recover useful energy from pressure drops occurring in the geo-fluid and cooling water in directcontact cycles.

\subsection{Heat Rejection Systems}

Very preliminary reviews of heat rejection systems were made which compared wet cooling towers, dry towers, water spray-augmented dry towers and evaporative condensers.

\subsection{Reference Cycles}

The last category of effort consists of the determination of cycle state points and power balances for four reference cycles consisting of:

(1) Dual-boiling, single-condensing, all direct-contact pentane cycle

(2) Dual-boiling, single-condensing, all direct-contact isobutane cycle

(3) Triple-boiling, direct-contact isobutane cycle with triple shell-and-tube condensing

(4) Triple-boiling, triple-condensing, shell-and-tube isobutane cycle.

Power balances for the reference direct-contact cycles included assessment of working-fluid recovery parasitics. The two triple-boiling cycles represent low-cost energy binary cycles appropriate for moderately low-temperature geothermal resources, which could be selected for construction today without major risk. 


\subsection{Sensitivity Analyses}

During the course of the study several sensitivity analyses were conducted, the results of which are integrated with the presentation of the reference cycles. These analyses include:

(1) Determination of the effect on the performance of several cycles by changing the boiler temperatures $10^{\circ} \mathrm{F}$

(2) Determination of the effects of reducing direct-contact boiler/heater, and condenser pinch points (minimum temperature difference between geothermal and working fluids).

\section{RESULTS AND CONCLUSIONS}

Tabular results of the cycle-screening effort are shown in Tables I and II. Working-fluid recovery parasitics for the triple-boiling isobutane direct-contact reference cycle are presented in Table III. A tabular summary of power gained through use of optional power-recovery expanders is presented as Table IV. Results of the preliminary review of heat-rejection systems are presented as Table V. Finally, state points and power balances for the several reference cycles analyzed are included as Tables VI through XIII.

Results and conclusions reached to date from the study can be summarized as follows:

(1) A major difference between the direct-contact and shell-and-tube cycles is the requirement, inherent in the direct-contact cycle, for additional components and corresponding parasitic power losses required for recovery of working fluid. Otherwise, the direct-contact cycle appears to perform generally the same as a comparable shell-and-tube cycle.

(2) Isobutane and pentane produce essentially equivalent net plant power (at the same geo-fluid flow) for direct-contact cycles having shell-and-tube condensers and wet cooling towers. If optional power recovery expanders are utilized, the isobutane plant produces slightly more power than the comparable pentane cycle (up to $8 \%$ ).

(3) For shell-and-tube cycles, isobutane consistently results in slightly more net plant power than does pentane (about $7 \%$ ).

(4) Mixed-hydrocarbon working fluids showed advantage in net plant power (at the same geo-fluid flow) over single fluids for both direct-contact and shell-and-tube dual-boiling cycles (up to $18 \%$ ), but require complete countercurrent heat-exchanger flow to achieve this advantage. The advantage shown by mixed fluids will decrease or disappear for tripleboiling cycles.

(5) Direct-contact condensing in combination with a wet cooling tower introduces major parasitic penalties and working-fluid recovery complications. For a direct-contact cycle having that combination, pentane produces more net plant power than does isobutane. The pentane cycle, however, is expected to produce somewhat less net plant power than is a corresponding isobutane cycle with shell-and-tube condensing.

(6) Triple boiling and condensing resulted in a gain in net plant power of about $20 \%$, for a given flow of geo-fluid, relative to cycles having dual boiling and single condensing.

(7) A practicable working-fluid recovery system for a direct-contact cycle at Raft River conditions consists of preflashing the geo-fluid, compressing and cooling the condenservent vapors, and flashing the spent geo-fluid through an expander before reinjection. The chemical systems reviewed were not shown to be competitive with the mechanical systems. 
(8) Preliminary assessment of cooling towers concluded that a conventional dry tower would impose significant power and cost penalties relative to a wet tower. Accordingly, the cycle comparisons for this study assumed use of wet towers; use of dry towers should be studied as a separate problem.

(9) An evaporative condenser appears to offer a significant thermodynamic advantage over either a wet or spray-augmented dry tower with a single condenser, and may be superior to a wet tower combined with triple shell-and-tube condensing.

(10) The overall conclusions reached are as follows:

a. Net plant power (at the same geo-fluid flow) is about the same for a direct-contact isobutane cycle having $5^{\circ} \mathrm{F}$ boiler/heater pinch points, shell-and-tube condensers, and optional power-recovery expanders, as for a comparable shell-and-tube cycle with $10^{\circ} \mathrm{F}$ boiler/heater pinch points.

b. An improvement of up to $19 \%$ in net plant power, relative to the first Raft River 5-MW dual-boiling pilot plant (at the same geo-fluid flow), can be provided by a triple-boiling, triple-condensing isobutane direct-contact cycle with a working-fluid loss of $30 \times 10^{-6}$ times the geo-fluid flow.

c. With moderately optimistic assumptions regarding the performance and economics of direct-contact components, a direct-contact binary cycle appears to have the potential for somewhat lower cost electrical energy than does a comparable shelland-tube cycle.

d. Work recommended before finalizing a second 5-MW Raft River Pilot Plant includes additional study of heat rejection systems, and economic assessment of the more promising direct-contact and shell-and-tube cycles. 


\section{DESCRIPTION OF THE CYCLES}

\section{BASIC BINARY CYCLES}

\subsection{Background}

For the basic geothermal binary Rankine cycle, the working fluid undergoes; first, a compression process at nearly constant entropy; second, a heat addition process at constant pressure; third, an expansion process through a turbine during which work is extracted at nearly constant entropy; and last, a heat rejection process during which the working fluid is condensed at constant pressure. The heat addition is accomplished in a heat exchanger where energy from the geothermal fluid is transferred to the working fluid. During the heat rejection phase, energy is extracted from the working fluid in a heat exchanger and delivered to cooling water, from which heat is rejected to the atmosphere. (With an evaporative condenser the cooling-water loop can be eliminated.) The heat exchangers can be shell-and-tube or direct-contact types.

Electrical power generation plants in which shell-and-tube heat exchangers are utilized, can be described as "shell-and-tube plants." If direct-contact heat exchangers are used for both heat addition and rejection, the cycle can be referred to as an "all-direct-contact cycle." Reference 1, which discusses various binary shell-and-tube plants, includes a schematic diagram of a shell-and-tube cycle as Figure 1.2-1. Figure 1 of this report shows basic cycle components of a dual-boiling, direct-contact plant.

\subsection{Multiple Boiling}

A simple binary cycle for a typical hydrocarbon working fluid such as isobutane would contain a single boiler/heater, a single turbine, and a single condenser (plus, of course, a cooling tower, pumps, etc.) as shown in Figure 1.2-1 of Reference 1. In order to improve the efficiency of the cycle by reducing thermodynamic irreversibilities occurring during heat addition, multiple boilers can be introduced. Figure 1 of this report shows a schematic of such a direct-contact cycle. A comparable shell-and-tube cycle would be arranged similarly, but would contain shell-and-tube heat exchangers rather than the direct-contact exchangers depicted in Figure 1.

The general thermodynamics of a dual-boiling isobutane cycle are illustrated in the T-Q (temperatureheat exchanged) diagram shown in Figure 2. In this diagram the temperatures of the geothermal and working fluids and the cooling water are shown as functions of the heat transferred to and from the working fluid as the fluids pass through the various components in the cycle. The average difference in temperature between the geo-fluid and working fluid in the boilers and heaters is a measure of the irreversibility introduced during the heat addition process. (Similar irreversibilities are introduced during the transfer of heat from the working fluid to the cooling water during heat injection.)

Substantial departure of the working-fluid temperature profile from that of the geo-fluid is caused by the constant-boiling temperature behavior of the isobutane. It becomes clear that the dual-boiling process has reduced the average temperature difference between fluids relative to the average difference for a single boiler. Similarly, the T-Q diagram shown in Figure 3, which illustrates the thermodynamics of a tripleboiling cycle, demonstrates a closer match between geothermal and working-fluid temperatures than does the dual-boiling cycle, and therefore shows a reduced irreversibility. The reduced irreversibilities of the multiple-boiling heat addition process can produce significant improvements in net geo-fluid effectiveness, which is defined as net plant output power per unit mass flow of geo-fluid (watt-hr/lbm geo-fluid).

\subsection{Supercritical Cycles}

A second approach to cycle improvement through reduction of irreversibilities, consists of adding heat to a working fluid without incurring boiling. In this way a small and relatively constant temperature difference can be maintained between the geothermal and working fluids, resulting in a reduced accumulation 
of irreversibility during heat addition. By adding heat at sufficiently high pressure, boiling can be avoided; working-fluid vapor is generated in a supercritical heating process.

Supercritical cycles have been evaluated at EG\&G Idaho, Inc., for several working fluids (Reference 1) including isobutane and propane. The net geo-fluid effectiveness was found to be lower for the supercritical cycles than for a dual-boiling isobutane cycle at Raft River geo-fluid conditions $\left(290^{\circ} \mathrm{F}\right)$ for all fluids investigated. The higher pressures required for the supercritical cycles resulted in higher workingfluid pumping losses for the shell-and-tube cycles considered. An even larger parasitic penalty would result for supercritical direct-contact cycles because of the necessity for higher geo-fluid pumping parasitics. For these reasons the net geo-fluid effectiveness of supercritical cycles has not been evaluated here. However, those cycles are perhaps simpler than the multiple-boiling cycles, and should be considered further when economic assessments are made.

\subsection{Mixed-Hydrocarbon Working Fluids}

Boiling of mixtures of hydrocarbon working fluids at constant pressure occurs over a temperature range, the magnitude of which varies with fluid mixture and boiling pressure. The T-Q diagram of Figure 4 illustrates the effect of this behavior in a dual-boiling cycle using a working-fluid mixture of half isobutane and half hexane. Heat addition irreversibilities for this cycle appear much reduced relative to the purefluid, dual-boiling cycle shown in Figure 2. This particular working fluid, however, exhibits a temperature change of about $70^{\circ} \mathrm{F}$ during constant-pressure condensation; this large temperature change does not match the assumed cooling-water temperature rise of $20^{\circ} \mathrm{F}$. The increased irreversibilities accumulated during heat rejection for this particular cycle more than offset the improved irreversibilities during heat addition (relative to the corresponding pure-fluid cycle), and the net geo-fluid effectiveness suffered. The proper mixed fluid would have the optimum balance between the heat addition and rejection effects.

\subsection{Multiple Condensing}

Condensing of a pure hydrocarbon working fluid at constant pressure occurs at constant temperature. A thermodynamic advantage exists in arranging multiple condensers in series (relative to cooling-water flow) for a cycle having multiple boilers and turbines. Figure 5 shows a schematic of a triple-boiling, triplecondensing cycle. It can be seen that for the same condenser pinch points, the same heat rejected, and the same cooling-water flow, the highest condensing temperature in the triple-condensing cycle will equal the condensing temperature for a comparable single-condensing cycle. The other two condensers will provide lower turbine exhaust pressures for two of the three turbines than would be provided by a single condenser. The result is increased plant power for the multiple-condensing cycle. The same conclusion is reached when looking at the reduced irreversibilities accumulated in the heat rejection part of the triple-condensing cycle (relative to a single condenser) as shown by comparing Figures 2 and 4 .

\section{DIRECT-CONTACT CYCLES}

\subsection{Comparison with Shell-and-Tube Cycles}

The direct-contact cycle, as envisioned for the present study, is much the same as the conventional shelland-tube binary cycle as presented in Reference 1, but with direct-contact boilers, preheaters, and perhaps condensers substituted for the corresponding shell-and-tube components. (Figures 1, 5, and 6 show schematics of three direct-contact cycles.) However, minor differences do exist between basic directcontact and shell-and-tube cycles.

Because of the existence of essentially a mechanical mixture of geothermal and working fluids in the direct-contact boilers, the vapor efflux contains both steam and working fluid. The total vapor pressure, can be approximated by the sum of the saturation pressures of working fluid and water at the boiling temperature. As a result, the direct-contact boiler pressures are higher than shell-and-tube boiler pressures 
(at least for low dissolved noncondensible content in the geo-fluid), and the turbines are driven by a mixture of a few percent by weight of steam along with the working fluid. Both working fluid and geo-fluid must be pumped to the boiler pressures in the direct-contact cycles.

Another difference results from the use of the direct-contact boilers in a multiple-boiling cycle. The high- and low-temperature boilers have different pressures, and therefore, the geo-fluid must undergo a pressure reduction as it passes from the high- to the low-temperature boiler. For the nominal cycle, throttling has been selected for this process, although some power gain can be derived through use of an expander at the expense of added plant complexity and cost.

\subsection{Working-Fluid Recovery Approaches}

2.2.1 Background. Although the basic direct-contact cycle is substantially the same as the basic shell-and-tube cycle, significant additions are required in a real plant to effect recovery of working fluid for the direct-contact cycle. The need for this recovery arises for three reasons:

(1) Working fluid has some solubility in geo-fluid, and unacceptable amounts of working fluid would be reinjected with the spent geo-fluid if some recovery were not accomplished.

(2) The geo-fluids, in general, have noncondensible gases dissolved when they enter the plants. Some of this dissolved gas will come out of solution in the direct-contact boilers, because the boiler pressure is less than the sum of the partial pressures of the water vapor, the working-fluid vapor, and the pressure of the noncondensible corresponding to its original concentration. In fact, for the cycles considered here, essentially all of the noncondensibles are expected to come out of solution in the high-pressure boiler, pass through the turbine, and enter the condenser. The presence of the noncondensibles in the condenser may well require condenser venting in order to reach the desired condenser pressure. Working-fluid loss arises because working-fluid vapor will be mixed with noncondensible gas in the condenser, and the mixed vapors would be vented together.

(3) Any working fluid which has dissolved in the cooling water in a direct-contact condenser will be discharged to the atmosphere in a wet cooling tower since the partial pressure of working fluid in the tower will be negligible relative to the air and water vapor pressures. (In addition, air will be dissolved in the cooling water during its exposure in the wet tower.) Recovery of the working fluid from the condenser cooling water must be addressed if that water is cooled in a wet cooling tower. A wet tower was assumed to be a component of the basic cycles examined in the present study, because preliminary considerations of cooling towers suggested that use of a conventional or spray-augmented dry tower places a higher power penalty on the cycle than does processing the cooling water to recover working fluid. Figure 5 is a schematic of a triple-boiling cycle with a shell-andtube condenser and with working-fluid recovery components. Figure 6 is a schematic of a dual-boiling cycle with a direct-contact condenser and a wet cooling tower; working-fluid recovery processes include cooling-water flashing.

2.2.2 Recovery of Working Fluid Dissolved in Geo-Fluid. Several approaches have been considered for the recovery of the working fluid dissolved in the geo-fluid ${ }^{2}$. Perhaps the most straightforward of these consists of flashing or expanding the geo-fluid, after it leaves the low-pressure heater, to a temperature (and pressure) which results in a reduced amount of working fluid remaining in solution. The working fluid which has come out of solution in the flashing process is mixed with steam, and must, in general, be pumped to the condenser pressure as a vapor mixture. Generally, no condensation occurs during this compression. The penalty for this recovery method is the power required for vapor compression and the extra geo-fluid pumping power which must be supplied to reinject from the reduced flash pressure. This recovery process has been quantitatively assessed in the present study, for both pentane and isobutane cycles. 
A second approach would be to sparge the geo-fluid by exposing it intimately to a noncondensible gas such as nitrogen or carbon dioxide. This process will cause a fraction of the working fluid to come out of solution at the sparging pressure, which consists of the partial pressures of working fluid, noncondensible, and water vapor. Henry's constant for the noncondensible and working fluid defines partial pressures for those gases; the partial pressure of steam is a function of the sparging temperature. The noncondensible would subsequently be separated from the mixture of working fluid and water vapors by refigeration, by compressing and cooling (working-fluid condensation) followed by a liquid-vapor separation, or by some vapor extraction process (see Section II-2.2.4).

A third approach for recovery of dissolved working fluid would consist of utilizing the same sparging technique, but subsequently passing the vapor mixture through a liquid hydrocarbon solvent such as paraxylene to dissolve the working fluid. The noncondensible gas and most of the water vapor would be separated mechanically and reused for continued sparging, leaving the working fluid dissolved in the solvent. The last step would be to separate the solution components by some distillation or preferential vaporization process.

A fourth method would consist of utilizing an adsorbent bed, such as charcoal, to adsorb the working fluid. Although the charcoal bed could accept liquid consisting of geo-fluid and dissolved working fluid, the magnitudes of liquid which would have to be handled by the bed would be large (the entire geo-fluid flow) for this process. An alternate use of the charcoal bed would be following a sparging process, whereby the bed would adsorb working fluid from a mixture of vapors resulting from the sparging. In either case, the working fluid would be recovered from the charcoal bed by application of heat. Some sort of batch process including switching of beds from the adsorbing to the desorbing mode would probably be required.

The second, third, and fourth approaches discussed here were considered, and judged not to be competitive with the first method (flashing the spent geo-fluid).

2.2.3 Recovery from Cooling Water. Recovery of working fluid from cooling water is necessary when a wet cooling tower is used to cool the water for a direct-contact condenser. A first category of solutions to this problem consists of isolation of the condenser from the atmosphere, thereby eliminating the need for recovery. Isolation can be accomplished by either elimination of the wet tower, adding a water loop between the direct-contact condenser and wet tower, or using a surface condenser in combination with a wet tower. Use of a surface condenser with a wet tower has been studied and is reported in Section IV. Use of evaporative condensers is promising and should be evaluated further (see Section IV-5.2).

The second category of solutions consists of recovery of the working fluid which is much the same as discussed in the previous section for recovery of working fluid dissolved in geo-fluid. Of those specific solutions considered, the approach quantitatively assessed for this study consists of flashing the cooling water after it has left the condenser, compressing the flashed vapors and discharging them into the condenser, pumping the flashed liquid cooling water to the pressure of the wet cooling tower (one atmosphere), and pumping the cooling tower discharge to the condenser pressure. It might be noted here that dissolved air will come out of solution when the cooling water is flashed and will be pumped into the condenser with the working-fluid vapor and steam. Accordingly, an additional load will be placed on condenser-vent vapor processing; the magnitude of this effect was estimated in the present evaluation.

2.2.4 Recovery from Condenser Vent. Recovery of working fluid from condenser vent vapors can be accomplished using several mechanical techniques. Perhaps the first method that comes to mind consists of refrigerating the vent vapor, so that further condensation of the working fluid will occur. The noncondensibles can then be vented to the atmosphere, accompanied by only a small fraction of the working fluid and steam which was vented from the main condenser. A trade-off. exists between turbine power: and: condenser-vent refrigeration power, because as the noncondensible partial pressure in the condenser increases, at a fixed condensing temperature, the ratio of noncondensible flow (which is fixed) to workingfluid vapor flow increases, the working-fluid vapor flow decreases, the refrigeration power decreases, and the turbine power decreases. Therefore an optimum condenser pressure must be found. This approach was 
evaluated for a pentane cycle using Raft River geo-fluid; for the conditions investigated, an icing limit $\left(32^{\circ} \mathrm{F}\right)$ was reached before sufficient recovery was accomplished. In many cases formation of hydrates of working fluids would be expected to occur before the icing limit is reached.

A second mechanical recovery method consists, in principle, of compressing the condenser-vent vapors to a higher pressure and then cooling and condensing the compressed mixture with cooling-tower water. Following the second condensation at the higher total pressure, a larger fraction of the vapor will be the noncondensible gas, than for the vapors initially leaving the main condenser. The extra cooling-tower load is very small, (a fraction of a degree Fahrenheit); the cycle penalty consists of the vapor compression power required. The same condenser-pressure optimization must be considered as mentioned in the preceding paragraph. A refinement was considered in this process. To avoid condensing working fluid in the vapor compressor, an effect which could result in compressor-performance degradation and perhaps blade deterioration, sufficient heat must be added before or during compression. For some calculations an inefficient pump ( $50 \%$ efficiency) was found to avoid condensation. A more efficient process for the cycle, however, consists of adding a small heater which utilizes spent geo-fluid energy to preheat the vapors to be compressed. This recovery method was studied in some detail both for isobutane and pentane cycles.

Chemical approaches to recovering working fluid from the condenser-vent vapors, reviewed briefly in the present effort, embody some of the same concepts which can be employed for dissolved working-fluid recovery. These approaches consist of: (a) passing the vent vapors through a solvent, separating and venting the noncondensibles, and then heating or distilling the solution of working fluid and solvent to recover the working fluid, (b) passing the condenser-vent vapors through an adsorbent bed, venting the noncondensibles, and heating the bed to recover the working fluid (this approach would probably require a batch process), and (c) passing the vent vapor through a liquid which serves as a strong getter of the noncondensible component of the mixture, returning the condensible components to the condenser, and finally driving the noncondensible out of the getter with heat from the geo-fluid. Lawrence Berkeley Laboratory (LBL) has investigated this latter approach for gettering carbon dioxide, present in the East Mesa geo-fluid, and has relegated the approach to a backup position for their 500-KW pilot plant. A comparable process for treating the nitrogen in the Raft River geo-fluid has not been found.

2.2.5 Preflashing the Geo-Fluid. A large fraction of the dissolved noncondensibles can be removed from the geo-fluid by preflashing, before it enters the high-pressure boiler. A small recovery of the power penalty due to flashing can be achieved by discharging the noncondensible gas and water-vapor mixture through an expander, and then venting to the atmosphere. The cycle power penalty for this approach consists of the additional pumping power required to pump the geo-fluid to the boiler pressure after flashing, the reduction in geo-fluid flow passing through the boiler, and the reduction of its enthalpy (relative to no preflash) after it has been pumped back to the boiler pressure. The latter two effects show up as changed cycle flow and state points, and therefore reduced turbine power at the same total geo-fluid flow and condenser pressure.

Except for the additional geo-fluid pumping power caused by the preflash, almost all of the penalty to the cycle can be eliminated by introducing a shell-and-tube heat exchanger to boil a few percent of the working fluid. The energy used for boiling is supplied by the vapors resulting from the preflash. Most of the water vapor condenses as the heat is exchanged; the condensate is fed into the boiler, and the remaining vapor, including the noncondensible, is vented to the atmosphere. This scheme has been evaluated, and is shown in Figure 5; it is essentially the same as that being used in the LBL 500-KW pilot plant.

\subsection{Optional Power-Recovery Expanders}

The pressure drops that occur in the geo-fluid for direct-contact cycles can be accomplished through hydraulic expanders rather than throttling orifices or valves. In this way some of the power can be recovered which is expended to pump the geo-fluid to the pressure of the high-pressure boiler. Part of the geo-fluid pressure drops are associated with the basic cycles, and part are associated with working-fluid recovery processes. The location and functions of the expanders can be visualized from the following two sections with the aid of Figures 5 and 6. 
2.3.1 Expanders in Basic Cycle. During the passage of geo-fluid from the higher to the lower pressure boilers/heaters in the direct-contact cycles, power-recovery expanders can be used in place of throttling orifices to accomplish the pressure drops. The isentropic efficiency of the hydraulic expander can be quite high in this application, since the geo-fluid remains a single-phase liquid for the process. Effects of enthalpy extracted from the cycle by the expanders are minimal. Use of the expanders was evaluated in the present study (Section IV-4.2).

2.3.2 Expanders in the Working-Fluid Recovery System. Four expanders were evaluated for recovering power from the working-fluid recovery system (Section IV-4.3):

(1) The vapors resulting from the preflash of geothermal fluid can be discharged to the atmosphere through a vapor expander. The power recovered was found to be small $(0.1 \%$ of net plant power in one case), and use of that expander probably would not result in a reduction of the cost of electricity.

(2) The actual preflash of geo-fluid can be accomplished through a hydraulic turbine rather than a throttling orifice. Power recovered in this way was also found to be small.

(3) Postflashing the spent geo-fluid before reinjection, to recover the dissolved working fluid, can be accomplished with an expander. This expander was found to recover a significant part of the working-fluid recovery parasitic losses.

(4) Flashing the cooling water through an expander rather than a throttling orifice can recover a significant part of the parasitic loss required by this working-fluid recovery. method. As previously: mentioned, flashing the cooling water is not necessary if the working-fluid condensation process is isolated from the atmosphere (such as if a shelland-tube condenser or a dry cooling tower is used). The power recoverable from this application of expanders has been assessed for two cycles in Sections IV-6.2 and IV-6.3. 


\title{
III. ANALYSIS METHODS USED FOR DIRECT-CONTACT, STATE POINT, AND POWER-BALANCE CALCULATIONS
}

\author{
1. GENERAL
}

\subsection{Basic Approach}

Before actually conducting the direct-contact cycle calulations, it was necessary to assemble specific calculational methods to be used for each of the cycle components. These methods generally employ steady state continuity and energy balances along with constitutive relationships, as they are appropriate, to express empirically determined behavior of the direct-contact components, such as realistic pinch point temperature differences. Turbine and compressor efficiencies were assigned to represent deviations in enthalpy changes from those of isentropic processes covering the pressure changes.

Dissolved working-fluid and noncondensible gas behavior was assumed to follow equilibrium processes as described by Henry's law. Mixtures of vapors were assumed to follow Dalton's law of partial pressures, which has ideal gas behavior implications in that each vapor component is assumed to occupy a volume independent of the molecules of the other vapors. Properties of water vapor and working-fluid vapors in mixtures were taken from tables representing the properties of the individual components, despite the fact that the deviation of an individual vapor component from ideal gas behavior suggests that its properties should not necessarily be expected to be preserved when that vapor is in the presence of molecules of another vapor. The approximations made, however, were judged to be consistent with, or better than, other uncertainties and assumptions contained in the analyses.

It should be pointed out that methods used for the shell-and-tube component and cycle calculations are not presented in this report; they are considered to be relatively standard and are broadly accepted.

\subsection{Alternate Methods for Treating Vapor Mixtures}

An interesting point relative to the treatment of flows of vapor mixtures arose during the calculations. Although data are not presently available at EG\&G Idaho, Inc., Professor Jacobs, University of Utah, has indicated that for his boiler experiments, the vapor mixture can be better described using ratios of specific volumes of the individual components evaluated at their saturated vapor pressures and the boiler temperature, than by the ratio of the same vapor pressures times the ratio of vapor molecular weights ${ }^{2}$. On the other hand, data for the pentane-water azeotrope at $100^{\circ} \mathrm{F}$ (Handbook of Chemistry and Physics, $51 \mathrm{st}$ Edition) showed almost complete agreement with the partial-pressure ratio method, whereas the specificvolume ratio method deviated about $5 \%$ from the data. Since the hand calculations of the present effort up to that time had used partial pressure ratios times molecular weight ratios to determine vapor flow ratios in a mixture, a cycle was redone using specific-volume ratios.

The effects of the assumptions chosen were evaluated for the boilers and turbines individually, and for the boiler-turbine combination in a dual-boiling isobutane cycle. It was interesting to find that the two methods predicted a noticeable difference in water vapor leaving the boilers ( $1 \%$ for the volume ratio method versus $2 \%$ for the pressure ratio method), but that the total turbine power was within about $2 \%$ for the two methods. (In fact, the partial pressure ratio method predicted $2 \%$ less turbine power for the same geo-fluid flow.) This comparison emphasized the fact that energy imbalances could be introduced by changing methods within a given cycle calculation, and that using the same method throughout the calculations is necessary. For completeness, both methods will be treated in succeeding sections; it can be seen that, generally, the specific-volume ratio method is more difficult to apply by hand, sometimes requiring an additional level of iteration for numerical solution. The calculations, for which the results are shown in this report, have, for the most part, been made using the ratio of partial pressures times the ratio of molecular weights to calculate vapor flow ratios in a vapor mixture. Exceptions to this are the cycles involving mixed-hydrocarbon working fluids and the triple-boiling, direct-contact isobutane cycle. 


\subsection{Simplified Method for Treating Azeotropes}

1.3.1 Background. In the direct-contact heat exchangers, geothermal and hydrocarbon working fluids are brought into intimate contact. Because of slight solubilities of hydrocarbon in water and of water in hydrocarbon, a relatively complex equilibrium phase relationship, which includes an azeotrope, exists for the fluids in the boilers/heaters and the condensers.

The question arises as to whether the actual equilibrium behavior of the azeotrope, which is present for nearly the entire range of compositions between pure water and pure hydrocarbon, can be adequately approximated by simple relationships readily amenable to analysis of the thermodynamic cycles. A more complete treatment would require that mixture properties be determined using equilibrium analyses as discussed by Katz ${ }^{6}$, or by methods incorporated in Thermodynamics Properties Program (THERPP) ${ }^{9}$, but expanded to include water as a component.

1.3.2 Simplified Calculational Method. It was reasoned that if water and working fluid were completely immiscible under equilibrium conditions, the vapor phase would exhibit partial pressures of water and working fluid which would be the same as for pure water and pure working fluid at the temperature of the mixture. To describe the actual vapor composition and total pressure, it was expected that the behavior of the real azeotrope might be well represented by partial pressures as determined for the individual components at the mixture temperature. Experimentally determined values of Henry's constants which describe the solubilities of water in working fluid and working fluid in water would be applied to calculate the hydrocarbon-rich and water-rich liquid compositions, respectively.

1.3.3 Checkout of Simplified Method. Sample equilibrium calculations were conducted by E. Hoffman, energy consultant, to determine the azeotrope pressures for pentane water mixtures at $100^{\circ} \mathrm{F}$ which approximated the conditions in both a shell-and-tube and a direct-contact condenser for a pentane geothermal cycle. The calculations used vapor pressures of the pure components, and Henry's constants for water dissolved in pentane and pentane dissolved in water, to determine the four equilibrium constants needed for the analysis. The equilibrium analyses resulted in total azeotrope pressures for the two mixtures which were equal, and which were in good agreement with the pressure as found from the simplified approach outlined in the preceding paragraph. In fact, for this example, the simplified approach produced a total pressure in almost exact agreement (within $0.1 \%$ ) with the pentane water azeotrope data mentioned in the preceding section. It was concluded that the simplified approach could safely be used in the present analyses to describe the behavior of the mixtures in direct-contact boilers and heaters as well as condensers.

\section{BOILER STATE POINT CALCULATION METHOD}

\subsection{General Approach and Assumptions}

The energy and continuity relationships have been applied to the direct-contact boiler calculations (see Figure 1 for a schematic sketch of a boiler) including the following assumptions and simplifications:

(1) The geo-fluid entering was assumed to have the properties of pure water.

(2) Energy and mass balances were made, initially, accounting for the working fluid dissolved in the geo-fluid. The corresponding additional terms were found to have negligible effect, so the numerical calculations were made neglecting them. The equations shown here do not include the dissolved working-fluid terms in the boiler mass and energy balances. (Calculations treating recovery of dissolved working fluid were made separately, and used Henry's law for determination of the quantitative relationships.)

(3) The geo-fluid entering the boiler is at boiler pressure, and at an enthalpy equal to that leaving the geo-fluid delivery pump. 
(4) The working fluid entering the boiler is at boiler temperature and pressure.

(5) The working-fluid vapor leaving the boiler is all saturated vapor at boiler temperature (no liquid leaves with the geo-fluid).

(6) The water vapor leaving the boiler is saturated vapor at boiler temperature.

(7) The geo-fluid leaving the boiler has a temperature higher than the boiler temperature by an amount equal to a pinch point temperature increment, $\Delta \mathrm{T}_{\mathrm{PB}}$.

\subsection{Pure Working Fluids}

The energy balance can be written (See Figure 1):

$$
w_{\text {GFin }} h_{\text {GFin }}+w_{\text {WFin }} h_{\text {WFin }}=w_{\text {GFout }} h_{\text {GFout }}+w_{\text {WFout }} h_{\text {WFout }}+w_{\text {STM }} h_{\text {STM }} \text { * }
$$

Mass balances are:

$$
\mathrm{W}_{\text {GFin }}=\mathrm{W}_{\text {GFout }}+\mathrm{W}_{\text {STM }} \quad ; \quad \mathrm{W}_{\mathrm{WFin}}=\mathrm{W}_{\text {WFout }}
$$

where

WWFout $_{\text {is saturated vapor at }} \mathrm{T}_{\mathrm{BOIL}}$

$\begin{array}{lll}\text { W } & \sim & \text { weight flow } \\ \text { T } & \sim & \text { temperature } \\ \text { Subscript GF } & \sim & \text { geo-fluid } \\ \text { Subscript WF } & \sim & \text { working fluid } \\ \text { Subscript STM } & \sim & \text { steam } \\ \text { Subscript in } & \sim & \text { going into boiler } \\ \text { Subscript out } & \sim & \text { leaving boiler } \\ \text { Subscript BOIL } & \sim & \text { boiler. }\end{array}$

The mass and energy balances can be combined to give:

$$
\frac{W_{\text {GFin }}}{W_{W F}}\left(h_{\text {GFin }}-h_{\text {GFout }}\right)-\frac{W_{\text {STM }}}{W_{W F}}\left(h_{\text {STM }}-h_{\text {GFout }}\right)=\left(h_{\text {WFout }}-h_{\text {WFin }}\right) \text {. }
$$

The terms in Equation (3) can be rewritten:

$$
\mathrm{h}_{\text {GFout }} \doteq \mathrm{h}_{\mathrm{GFSL}} \text { at } \mathrm{T}_{\text {BOIL }}+\Delta \mathrm{T}_{\mathrm{PB}}+\mathrm{v}_{\mathrm{GF}} \mathrm{P}_{\mathrm{WF}}
$$

where

$$
\begin{array}{lll}
\mathrm{h}_{\mathrm{GFSL}} \text { at } \mathrm{T}_{\mathrm{BOIL}} & \sim \quad \text { enthalpy of saturated liquid water at the boiler temperature } \\
\mathrm{V}_{\mathrm{GF}} & \sim \quad \text { specific volume of liquid water at } \mathrm{T}_{\mathrm{BOIL}} \\
\mathrm{P}_{\mathrm{WF}} & \sim \quad \text { partial pressure of working-fluid vapor in boiler. }
\end{array}
$$


The specific heat of liquid water has been taken as unity in Equation (4).

$$
\frac{\mathrm{W}_{\mathrm{STM}}}{\overline{\mathrm{W}}_{\mathrm{WF}}}=\frac{\mathrm{P}_{\mathrm{STM}}}{\mathrm{P}_{\mathrm{WF}}} \frac{\mathrm{M}_{\mathrm{STM}}}{\mathrm{M}_{\mathrm{WF}}}
$$

where

$$
\begin{aligned}
\text { P }_{\mathrm{STM}} & \sim \text { partial pressure of steam in boiler } \\
\mathrm{M} & \sim \text { molecular weight } \\
\mathrm{h}_{\mathrm{STM}}-\mathrm{h}_{\mathrm{GFOut}} & =\left(\mathrm{h}_{\mathrm{STM}}-\mathrm{h}_{\mathrm{GFSL}} \text { at } \mathrm{T}_{\mathrm{BOIL}}\right)+\left(\mathrm{h}_{\mathrm{GFSL}} \text { at } \mathrm{T}_{\mathrm{BOIL}}-\mathrm{h}_{\mathrm{GFout}}\right) \\
& =\mathrm{h}_{\mathrm{Fg}_{\mathrm{GF}} \text { at } \mathrm{T}_{\mathrm{BOIL}}}-\mathrm{v}_{\mathrm{GF}} \mathrm{P}_{\mathrm{WF}}-\Delta \mathrm{T}_{\mathrm{PB}}
\end{aligned}
$$

where

$\mathrm{h}_{\mathrm{fgGF}}$ at $\mathrm{T}_{\mathrm{BOIL}} \sim$ heat of vaporization of water at boiler temperature ${ }^{v_{G F P W F}}$ is small relative to $\mathrm{h}_{\mathrm{fg}_{\mathrm{GF}}}$, and has been neglected.

For pure working fluids:

$$
\begin{aligned}
h_{\text {WFout }}-\mathrm{h}_{\text {WFin }} & =\left(\mathrm{h}_{\text {WFout }}-\mathrm{h}_{\text {WFSL at }} \mathrm{T}_{\text {BOIL }}\right)+\left(\mathrm{h}_{\text {WFSL at }} \mathrm{T}_{\text {BOIL }}-\mathrm{h}_{\text {WFin }}\right) \\
& \doteq \mathrm{h}_{\mathrm{fg}_{\text {WF }} \text { at } \mathrm{T}_{\text {BOIL }}-\mathrm{v}_{\text {WFL }} \mathrm{P}_{\text {STM }}}
\end{aligned}
$$

where

$\mathrm{v}_{\mathrm{WFL}} \sim \quad$ specific volume of liquid working fluid.

Substituting Equations (5-7) into Equation (3), the final direct-contact boiler balance becomes:

$$
\frac{W_{\text {GF in }}}{W_{W F}}=\frac{h_{f g_{W F}}-v_{\text {WFL }} P_{S T M} \frac{144}{788}+\frac{{ }_{S T M}}{P_{W F}} \frac{{ }_{S T M}}{M_{W F}}\left(h_{f g_{G F}}-\Delta T_{P B}\right)}{h_{G F i n}-h_{G F o u t}} .
$$

In Equation (8), the conversion $\frac{144}{778}$ has been included to effect consistency for $h$ in $\mathrm{Btu} / \mathrm{lbm}, \mathrm{v}$ in $\mathrm{ft}^{3} / \mathrm{lbm}$, and $\mathrm{P}$ in psia; the specific heat of geo-fluid has been taken as unity.

Note that $h_{\text {GFout }}$ can be evaluated at $\left(\mathrm{T}_{\mathrm{BOIL}}+\Delta \mathrm{T}_{\mathrm{PB}}\right)$ and the boiler pressure, $\left(\mathrm{P}_{\mathrm{STM}}+\mathrm{P}_{\mathrm{WF}}\right)$. 
The geo-fluid leaving the boiler can be calculated:

$$
\frac{W_{\text {GFout }}}{W_{W F}}=\frac{W_{\text {GFin }}}{W_{W F}}-\frac{W_{\text {STM }}}{W_{W F}} .
$$

Equation (5) has used the partial pressure ratio method for calculation of vapor flows. Using the ratio of specific volumes, Equation (5) can be rewritten as:

$$
\frac{{ }_{\text {STM }}}{W_{W F}}=\frac{v_{W F V}}{{ }_{\text {STM }}}
$$

where

$$
\begin{aligned}
& \mathrm{v}_{\mathrm{WFV}} \sim \quad \begin{array}{l}
\text { specific volume of working-fluid vapor; for the boiler the vapor is saturated } \\
\text { at } \mathrm{T}_{\mathrm{BOIL}}
\end{array} \\
& \mathrm{v}_{\mathrm{STM}} \sim \quad \text { specific volume of saturated steam at } \mathrm{T}_{\mathrm{BOIL}} .
\end{aligned}
$$

Equation (8) can be rewritten using the ratio of specific volumes to calculate vapor flow ratios as:

$$
\frac{W_{\text {GFin }}}{W_{W F}}=\frac{{ }_{f g_{W F}}-v_{W F L} P_{S T M} \frac{144}{788}+\frac{v_{W F V}}{v_{S T M}}\left(h_{f g_{G F}}-\Delta T_{P B}\right)}{h_{\text {GF in }}-h_{\text {GF out }}}
$$

Equation (8) or ( $8 \mathrm{a}$ ) can be solved after a boiler temperature has been selected, and a boiler pinch point, $\Delta \mathrm{T}_{\mathrm{PB}}$, assumed.

Introduction of the geo-fluid preflash and the shell-and-tube boiler which is supplied with energy from the preflash vapors, as shown for the cycle in Figure 5, requires a slight modification to the boiler mass and energy balance. Part of the working fluid leaving the high-pressure heater is evaporated in the shell-andtube boiler before being returned to the direct-contact boiler. The resulting direct-contact boiler balance becomes:

$$
\begin{aligned}
& \frac{W_{\text {GFin }}}{W_{\text {WF out }}}=\frac{\left(h_{f_{\text {WF }}}-v_{\text {WFL }} P_{\text {STM }} \frac{144}{788}\right)\left(1-\frac{W_{\text {WFE }}}{W_{\text {WFout }}}\right)}{h_{\text {GF in }}-h_{\text {GF out }}} \\
& -\frac{\frac{W_{\text {WFE }}}{W_{\text {WFout }}}\left(h_{\text {WF SWV }} \propto P_{\text {BOIL }}-h_{\text {WF SV }} \propto T_{\text {BOIL }}\right)}{h_{\text {GFin }}-h_{\text {GFout }}} \\
& +\frac{\left.\left.\frac{\mathrm{W}_{\text {STM }}}{\mathrm{W}_{\text {WFout }}}\right|_{\mathrm{fg}_{\mathrm{GF}}}-\Delta \mathrm{T}_{\mathrm{PB}}\right)}{\mathrm{h}_{\text {GFin }}-\mathrm{h}_{\text {GFout }}}
\end{aligned}
$$


where
WWFE
$\sim$ weight flow of working fluid evaporated in the shell-and-tube boiler
hWFSV@PBOIL
and $h_{W F S V} @ T_{B O I L}$
$\sim$ enthalpy of working-fluid saturated vapor at the total pressure and temperature of the high-pressure boiler.

But:

$$
\frac{W_{\text {WFE }}}{W_{\text {WFout }}}=\frac{W_{\text {WFE }}}{W_{\text {GFin }}} \frac{W_{\text {GFin }}}{W_{\text {WFout }}} \text {, }
$$

where $\frac{W_{\text {WFE }}}{W_{\text {GFin }}}$ can be found from an energy balance for the shell-and-tube boiler. Equations (10) and (11) can easily be solved simultaneously by iterative substitution.

\subsection{Mixed-Hydrocarbon Working Fluids}

Analysis of cycles using mixed-hydrocarbon working fluids requires additional modifications to the boiler balance (Equation 8 or $8 \mathrm{a}$ ). The corresponding equation for the high-pressure boiler can best be used in the form:

$$
\frac{W_{\text {GFin }}}{W_{\text {WF }}}=\frac{h_{\text {WFout }}-h_{\text {WF in }}+\frac{W_{\text {STMout }}}{W_{\text {WF }}}\left(h_{\text {STMout }}-h_{\text {GFout }}\right)}{h_{\text {GF in }}-h_{\text {GFout }}} .
$$

For the low-pressure boiler of a dual-boiling, mixed hydrocarbon cycle the entire working fluid from the low-pressure heater enters the boiler. The working-fluid split occurs following partial boiling in the lowpressure boiler. Liquid working fluid (not yet boiled) would be separated from the low-pressure boiler and pumped to the high-pressure heater. In other respects, heat addition would be accomplished as is shown in Figure 1 or 5. For mixed-hydrocarbon working fluids the low-pressure boiler balance can be written:

$$
\begin{gathered}
\frac{W_{\text {GFin }}}{W_{\text {WFin }}}=\frac{h_{\text {WFLout }}-h_{\text {WFin }}+\frac{W_{\text {STMout }}}{W_{\text {WFVout }}} \frac{W_{\text {WFVout }}}{W_{\text {WFin }}}\left(h_{\text {STMout }}-h_{\text {GFout }}\right)}{h_{\text {GFin }}-h_{\text {GFout }}} \\
+\frac{\frac{W_{\text {WFVout }}}{W_{\text {WFin }}}\left(h_{\text {WFVout }}-h_{\text {WFLout }}\right)}{h_{\text {GFin }}-h_{\text {GFout }}}
\end{gathered}
$$

where the subscripts WFLout and WFVout refer to the working fluid leaving the low-pressure boiler in the liquid and vapor states, respectively. 
For a given working-fluid mixture $h_{\text {WFLout }}, h_{\text {WFVout }}$, $h_{\text {STMout }}$, and $\frac{\mathrm{W}_{\text {FVout }}}{\mathrm{W}_{\text {WFin }}}$ are functions of the boiler pressure and final boiling temperature (dew-point temperature). $\mathrm{h}_{\mathrm{GFout}}$ is equal to the enthalpy of water at the boiler pressure and the temperature ( $\left.T_{\text {WFin }}+\Delta T_{P B}\right) \cdot \frac{W_{\text {STMout }}}{W_{\text {WFV }} \text { can }}$ can found as a function of the boiler pressure and temperature, using either the specific-volume or partialpressure ratio methods; however, for working-fluid mixtures the specific-volume ratio method seems more straightforward since the molecular weight of the working-fluid vapor does not remain fixed during the boiling process.

The working-fluid properties for the mixtures were determined as functions of temperature and working-fluid partial pressure using a modified version of THERPP, (see Reference 9) a computer program based on methods outlined in Reference 4 .

A final topic should be discussed pertaining to the calculation of boiler (and condenser) behavior for the mixed hydrocarbons in the direct-contact heat exchangers. The assumption was made that saturated water vapor, saturated working-fluid vapor, and saturated liquid working fluid exist simultaneously at a given location in the direct contactor, and have a temperature different from that of the geo-fluid at the same location. It follows, then, that at a given location in the boiler, the total boiler pressure is equal to the sum of the saturation pressures of water and working-fluid vapors for the working-fluid temperature at that location. Further, the assumption was made that the total boiler pressure is uniform throughout the boiler. Since the working-fluid temperature varies during boiling for mixed fluids, the partial pressure of steam also varies. An iteration level is thus introduced into the calculation to obtain compatible conditions connecting the bubble point (boiling starts), dew point (boiling complete), and intermediate two-phase conditions of the working fluid. These assumptions and corresponding calculational procedures are consistent with the experimental behavior for single-fluid, direct-contact boiling as presented in Reference 2 .

\section{HEATER CALCULATIONAL METHOD}

The simplifications and assumptions made for the direct-contact heater analysis procedure render the energy and mass balances very simple.

These assumptions are as follows:

(1) Complete separation of the fluids entering and leaving is maintained.

(2) Dissolved working fluid is assumed to have negligible effect on the mass and energy balances.

(3) The pinch point was found to exist at the working-fluid outlet for all of the conditions analyzed; calculations of internal conditions within the heater were not made. Note that for the assumptions made in these cycle calculations, the heater pinch point equals that for the boiler.

The energy balance is:

$$
W_{\text {GFin }} h_{\text {GFin }}+W_{\text {WFin }} h_{\text {WFin }}=W_{\text {GFout }} h_{\text {GFout }}+W_{\text {WFout }} h_{\text {WFout }} \cdot
$$

Mass balances are:

$$
W_{\text {GFin }}=W_{\text {GFout }} ; W_{\text {WFin }}=W_{\text {WFout }} \text {. }
$$


The resulting equation for heater analysis becomes:

$$
h_{\text {GFout }}=h_{\text {GFin }}-\frac{W_{W F}}{W_{G F}} \quad\left(h_{\text {WFout }}-h_{\text {WFin }}\right) .
$$

In application of Equation (16):

$\mathrm{h}_{\mathrm{GFin}}$ for the heater is assumed to equal $\mathrm{h}_{\mathrm{GFout}}$ for the boiler.

$\frac{\mathrm{W}_{\mathrm{WF}}}{\mathrm{W}_{\mathrm{GF}}}$ has been determined from the boiler calculation.

$h_{\text {WFout }}$ is the enthalpy of liquid working fluid at the boiler temperature and pressure.

$\mathrm{h}_{\text {WFin }}$ is the enthalpy of the working fluid entering the heater, and can be found for the high-pressure heater as a function of the low-pressure heater outlet temperature and pressure (for a dual- boiling cycle), plus the enthalpy increment supplied by the high-pressure working-fluid feed pump. For the low-pressure heater, $h_{W F i n}$ is found as a function of the condensing temperature and condenser pressure plus the increment in enthalpy required to pump the working fluid to the low-pressure heater from the condenser.

\section{CALCULATIONAL METHODS FOR TURBINES, LIQUID PUMPS, AND VAPOR COMPRESSORS}

\subsection{Turbines or Expanders}

For the present turbine calculations the presence of noncondensibles was assumed to have a negligible effect on the turbine expansion process and corresponding power balance, but was accounted for as it influences turbine exhaust pressure. Calculation of an isentropic process for the combined water and working-fluid flow through the turbine was made first to find an isentropic $\Delta \mathrm{h}$. The actual $\Delta \mathrm{h}$ was assumed to equal the isentropic $\Delta \mathrm{h}$ times the turbine efficiency. Turbine power was calculated later in the cycle analysis, after flow balances for the cycle were completed.

$$
\text { Turbine Power }(\text { megawatts })=\mathrm{W} \Delta \mathrm{h}_{\mathrm{ACT}} \times 0.29293 \times 10^{-6}
$$

where

$$
\begin{array}{ll}
\mathrm{W} & \sim \quad \text { turbine flow } \frac{\mathrm{lbm}}{\mathrm{hr}} \\
\Delta \mathrm{h}_{\mathrm{ACT}} \sim & \sim \text { actual enthalpy change through turbine, } \frac{\mathrm{Btu}}{\mathrm{lbm}}
\end{array}
$$

A stepwise calculation of the isentropic process through the turbine best presents the calculation method, and proceeds as follows:

(1) Calculate turbine inlet properties:

$$
h_{\text {in }}=\frac{h_{\text {STMin }} \frac{W_{\mathrm{H}_{2} \mathrm{O}}}{W_{W F}}+h_{W F i n}}{1+\frac{W_{H_{2} O}}{W_{W F}}}
$$




$$
s_{\text {in }}=\frac{s_{\text {STMin } \frac{W_{\mathrm{H}_{2} \mathrm{O}}}{W_{W F}}+s_{W F i n}}}{1+\frac{{ }_{\mathrm{H}_{2} O}}{W_{W F}}}
$$

where

$$
\begin{array}{lll}
\mathrm{h} & \sim & \text { enthalpy } \\
\mathrm{s} & \sim \text { entropy } \\
\frac{\mathrm{W}_{\mathrm{H}_{2} \mathrm{O}}}{\mathrm{W}_{\mathrm{WF}}} \sim \quad \text { ratio of water (steam) to working fluid leaving boiler. }
\end{array}
$$

Subscripts are defined as:

$$
\begin{array}{ll}
\text { STMin } & \sim \quad \text { property of saturated steam at the boiler temperature } \\
\text { WFin } & \sim \begin{array}{l}
\text { property of saturated working-fluid vapor at boiler } \\
\text { temperature }
\end{array} \\
\text { in } & \sim \text { property of fluid mixture at turbine inlet. }
\end{array}
$$

Note that for a mixture of ideal gases, entropy of mixing does not need to be included explicitly if each component's entropy is evaluated at its temperature and partial pressure.

(2) Guess an isentropic turbine exhaust temperature, $\mathrm{T}_{\mathrm{EX}}$

(3) Find PSTM and $v_{S T M}$ (saturated vapor properties) as functions of $\mathrm{T}_{\mathrm{EX}}$ from water properties. Since, in general, some water condenses, $\mathrm{P}_{\mathrm{STM}}=\mathrm{P}\left(\mathrm{T}_{\mathrm{EX}}\right)$.

(4) Find $\mathrm{P}_{\mathrm{WF}}=\mathrm{P}_{\mathrm{COND}}-\mathrm{P}_{\mathrm{STM}}$ at the turbine exit

where

$P_{C O N D}$ is the condenser pressure, and must include the partial pressures of the noncondensibles in the condenser.

(5) Find swF and ${ }^{v} W F$ (isentropic) at the turbine exit $=\mathrm{s}\left(\mathrm{T}_{\mathrm{EX}}, \mathrm{P}_{\mathrm{WF}}\right)$ and $\mathrm{v}\left(\mathrm{T}_{\mathrm{EX}}, \mathrm{P}_{\mathrm{WF}}\right)$.

(6) Find the entropy of water at the turbine exit, $\mathrm{s}_{\mathrm{H}_{2}} \mathrm{O}$.

$$
s_{\mathrm{H}_{2} \mathrm{O}}=\frac{s_{\text {in }}\left(1+\frac{\mathrm{W}_{\mathrm{H}_{2}}}{\mathrm{~W}_{\mathrm{WF}}}\right)-s_{W F}}{\frac{\mathrm{W}_{2} \mathrm{O}}{\mathrm{W}_{\mathrm{WF}}}}
$$

for an isentropic process of the total flow. 
(7) Find the quality of water (isentropic) at the turbine exit, $\mathrm{X}_{\mathrm{H}_{2}} \mathrm{O}$.

$$
\mathrm{x}_{\mathrm{H}_{2} \mathrm{O}}=\mathrm{x}\left(\mathrm{T}_{\mathrm{EX}}, \mathrm{s}_{\mathrm{H}_{2} \mathrm{O}}\right) \text {. }
$$

(8) Find the ratio of water vapor to working fluid at the turbine exit, $\frac{W_{S T M o u t}}{W_{W F}}$.

$$
\frac{\mathrm{W}_{\text {STMout }}}{\mathrm{W}_{\mathrm{WF}}}=\frac{\mathrm{W}_{\mathrm{H}_{2} \mathrm{O}}}{\mathrm{W}_{\mathrm{WF}}}\left(\mathrm{x}_{\mathrm{H}_{2} \mathrm{O}}\right) \text {. }
$$

(9) Check $\frac{\text { WSTMout }}{W_{W F}}$ against $\frac{\text { PSTM }}{P_{W F}} \frac{M_{S T M}}{M W F}$ or $\frac{v_{W F}}{v_{S T M}}$ depending on whether the partial-pressure ratio method or the specific-volume ratio method is assumed.

(10) If Step (9) does not check, select another $\mathrm{T}_{\mathrm{EX}}$ and repeat Steps (3) through (8) until Step (9) is satisfied.

(11) Calculate the isentropic turbine exhaust enthalpy:

$$
h_{\text {EXIS }}=\frac{h_{\mathrm{H}_{2} 0} \frac{\mathrm{W}_{\mathrm{H}_{2} \mathrm{O}}}{\mathrm{W}_{\mathrm{WF}}}+\mathrm{h}_{\mathrm{WF}}}{1+\frac{\mathrm{W}_{\mathrm{H}_{2} \mathrm{O}}}{\mathrm{W}_{W F}}}
$$

where

$$
\begin{aligned}
& \mathrm{h}_{\mathrm{H}_{2} \mathrm{O}}=\mathrm{h}\left(\mathrm{X}_{\mathrm{H}_{2} \mathrm{O}}, \mathrm{T}_{E X}\right) \\
& \mathrm{h}_{W F}=\mathrm{h}\left(\mathrm{P}_{W F}, \mathrm{~T}_{E X}\right) .
\end{aligned}
$$

(12) Calculate the isentropic enthalpy change; $\Delta \mathrm{h}_{\mathrm{IS}}=\mathrm{h}_{\text {in }}-\mathrm{h}_{\text {EXIS. }}$

\subsection{Liquid Pumps}

Enthalpy changes produced by liquid pumps in units of Btu/lbm were calculated as:

$$
\Delta h_{\text {ACT }}=\frac{v \Delta \mathrm{P} \times 144}{\eta_{\mathrm{P}} \times 778}
$$

where

$$
\begin{aligned}
& \mathrm{v}=\text { the liquid specific volume }\left(\frac{\mathrm{ft}^{3}}{\mathrm{lbm}}\right) \\
& \Delta \mathrm{P}=\text { the pressure rise through the pump (psia) } \\
& \eta_{\mathrm{p}}=\text { the pump efficiency. }
\end{aligned}
$$




\subsection{Vapor Compressors}

For the calculations reported here, vapor compression processes were forced to avoid condensation of any vapor component. Either heat was added preceding the compression as required, or a sufficiently low compression efficiency was assumed. The calculations reported were made assuming that the vapor flow ratios in the mixture could be expressed as:

$$
\frac{\mathrm{W}_{1}}{\mathrm{~W}_{2}}=\frac{\mathrm{P}_{1}}{\mathrm{P}_{2}} \frac{\mathrm{M}_{1}}{\mathrm{M}_{1}}
$$

where subscripts refer to vapor components.

With no condensation, the relationship between vapor flows and total pressure, $P$, at the end of compression is:

$$
P=P_{1}\left(1+\frac{W_{2}}{W_{1}} \frac{M_{1}}{M_{2}}+\frac{W_{3}}{W_{1}} \frac{M_{1}}{M_{3}}\right)
$$

where $P_{1}$ is the partial pressure of the saturated vapor component at the end of compression.

Since the flow ratios are fixed and known for the compression, the final total pressure can be found easily for an assumed final temperature if one of the components is a saturated vapor at that temperature. (A comparable relationship, assuming that specific volume ratios are inversely proportional to flow ratios, would involve an additional level of iteration.)

For a vapor compression process, where one component is a saturated vapor at the end of compression, and where the flows and the state point before compression are known, the procedure is as follows:

(1) Calculate compressor inlet enthalpy and entropy for the total flow.

(2) Guess a compressor discharge isentropic temperature.

(3) Find the discharge partial pressure of the saturated vapor component as a function of the isentropic discharge temperature.

(4) Find the discharge total pressure and each partial pressure.

(5) Find the discharge total entropy of the mixture for the assumed discharge temperature.

(6) Repeat Steps (2) through (5) until the entropy of Step (5) is equal to the entropy of Step (1). This is the final discharge state of the isentropic process.

(7) Knowing the discharge partial pressure and temperature of each component for the isentropic process, calculate the final total enthalpy, and isentropic $\Delta \mathrm{h}$.

The actual $\Delta \mathrm{h}$ is the isentropic $\Delta \mathrm{h}$ divided by the compressor efficiency. 


\section{DIRECT-CONTACT CONDENSER CALCULATIONAL METHOD}

\subsection{Basic Method for a Pure Working Fluid}

The basic approach for assembling a state point calculation method for the direct-contact condenser for pure working fluids starts with an overall mass and energy balance, as was done for the boiler. A schematic of the condenser is included in Figure 1. The following assumptions and simplifications have been included to provide constitutive relationships required to complete the method:

(1) Separation of condensed working fluid and water is assumed to have been accomplished within the condenser unit.

(2) Mutual solubilities of water and working fluid have been neglected in the mass and energy balances (based on a study made for the boiler).

(3) Solubility of noncondensibles in cooling water and working fluid in the condenser have been neglected in this section. When vapor mixtures from flashed cooling water are discharged into the condenser, and solubilities of noncondensibles within the condenser are included, some modification of these equations is needed to calculate the required cooling-water flow.

(4) The working-fluid vapor and noncondensibles vented from the condenser are assumed to leave at the condensing temperature, $\mathrm{T}_{\mathrm{C}}$.

(5) Condensing is assumed to occur at constant pressure, and the condensed working fluid is assumed to leave at the condensing temperature. (Condensing occurs at constant temperature for a pure fluid condensing at constant pressure.)

(6) The cooling water is assumed to leave the condenser at a pinch point increment in temperature, $\Delta \mathrm{T}_{\mathrm{PC}}$, below the condensing temperature.

Referring to Figure 1, mass balances can be written:

$$
\begin{aligned}
& \mathrm{W}_{\mathrm{H}_{2} \mathrm{Oin}}+\mathrm{W}_{\text {STMin }}=\mathrm{W}_{\mathrm{H}_{2} \text { Oout }}+\mathrm{W}_{\text {STMout }} \\
& \mathrm{W}_{\text {WFVin }}=\mathrm{W}_{\text {WFVout }}+\mathrm{W}_{\text {WFLout }} \\
& \mathrm{W}_{\mathrm{NCin}}=\mathrm{W}_{\text {NCout }}=\mathrm{W}_{\mathrm{NC}}
\end{aligned}
$$

where subscripts have the following definitions:

$\begin{array}{lll}\mathrm{H}_{2} \mathrm{O} & \sim & \text { liquid water } \\ \mathrm{STM} & \sim & \text { steam } \\ \mathrm{WFL} & \sim & \text { liquid working fluid } \\ \mathrm{WFV} & \sim & \text { working-fluid vapor } \\ \mathrm{NC} & \sim & \text { noncondensibles } \\ \text { in } & \sim & \text { entering condenser } \\ \text { out } & \sim & \text { leaving condenser. }\end{array}$


The energy balance can be written:

$$
\begin{aligned}
& \mathrm{W}_{\mathrm{H}_{2} \text { Oin }} \mathrm{h}_{\mathrm{H}_{2} \mathrm{Oin}}+\mathrm{w}_{\text {STMin }} \mathrm{h}_{\text {STMin }}+\mathrm{w}_{\text {WFVin }} \mathrm{h}_{\text {WFVin }}=\mathrm{w}_{\text {WFLout }} \mathrm{h}_{\text {WFLout }} \\
& +\mathrm{w}_{\text {STMout }} \mathrm{h}_{\text {STMout }}+\mathrm{w}_{\text {WFVout }} \mathrm{h}_{\text {WFVout }}+\mathrm{w}_{\mathrm{H}_{2} \text { Oout }} \mathrm{h}_{\mathrm{H}_{2} \text { Oout. }}
\end{aligned}
$$

The condenser pressure, $\mathrm{P}_{\mathrm{C}}$, can be written as:

$$
P_{C}=P_{S T M}+P_{W F V}+P_{N C} .
$$

Equations (27) and (28) can be combined to give:

$$
\begin{aligned}
h_{\mathrm{H}_{2} \mathrm{Oin}}-\mathrm{h}_{\mathrm{H}_{2} \mathrm{Oout}} & +\frac{\mathrm{W}_{\text {STMin }}}{\mathrm{W}_{\mathrm{H}_{2} \mathrm{Oin}}}\left(\mathrm{h}_{\text {STMin }}-\mathrm{h}_{\mathrm{H}_{2} \text { Oout }}\right) \\
& +\frac{\mathrm{W}_{\text {WFVin }}}{\mathrm{W}_{\mathrm{H}_{2} \mathrm{Oin}}}\left(\mathrm{h}_{\text {WFVin }}-\mathrm{h}_{\text {WFLout }}\right) \\
& =\frac{\mathrm{W}_{\text {WFVout }}}{\mathrm{W}_{\mathrm{H}_{2} \text { Oin }}}\left(\mathrm{h}_{\text {WFVout }}-\mathrm{h}_{\text {WFLout }}\right) \\
& +\frac{\mathrm{W}_{\text {STMout }}}{\mathrm{W}_{\mathrm{H}_{2} \text { Oin }}}\left(\mathrm{h}_{\text {STMout }}-\mathrm{h}_{\mathrm{H}_{2} \text { Oout }}\right) .
\end{aligned}
$$

Terms in Equation (30) can be rewritten:

$$
\begin{aligned}
& \left(\mathrm{h}_{\mathrm{H}_{2} \text { Oin }}-\mathrm{h}_{\mathrm{H}_{2} \text { Oout }}\right)=\mathrm{C}_{\mathrm{PH}_{2} \mathrm{O}}\left(\mathrm{T}_{\mathrm{H}_{2} \text { Oin }}-\mathrm{T}_{\mathrm{H}_{2} \text { Oout }}\right) \\
& \left({ }^{\mathrm{T}} \mathrm{C}-\mathrm{T}_{\mathrm{H}_{2} \text { Oout }}\right)=\Delta \mathrm{T}_{\mathrm{PC}}
\end{aligned}
$$

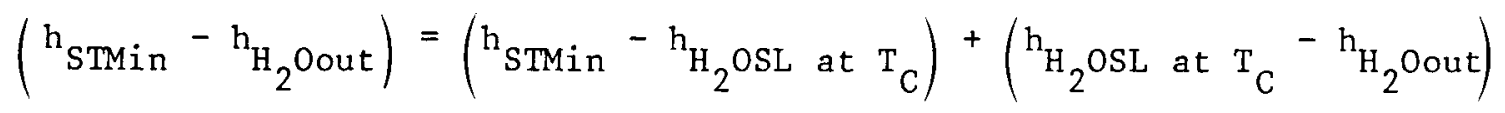

but:

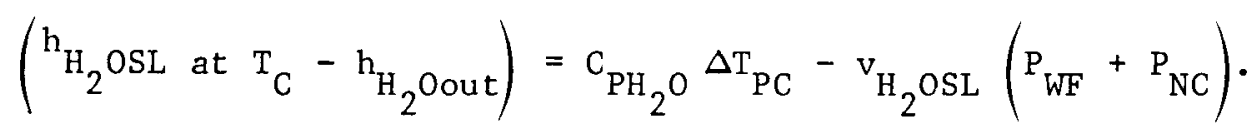


$\mathrm{C}_{\mathrm{PH}_{2} \mathrm{O}}$ is the specific heat of liquid water (and equals $\left.1.0 \frac{\mathrm{Btu}}{\mathrm{lb}^{\circ} \mathrm{F}}\right) ; \quad v_{\mathrm{H}_{2} \mathrm{OSL}} \quad\left(\mathrm{P}_{\mathrm{WF}}+\mathrm{P}_{\mathrm{NC}}\right)$ is small compared to $\Delta \mathrm{T}_{\mathrm{PC}}$, and will be neglected.

Then:

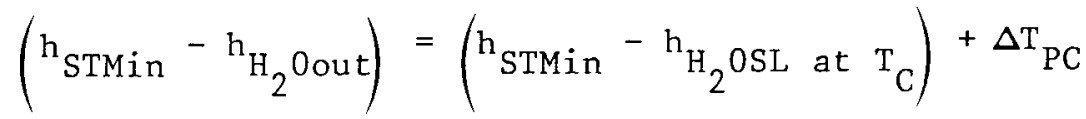

$$
\begin{aligned}
& \left(h_{\text {WFVin }}-h_{\text {WFLout }}\right)=\left(h_{\text {WFVin }}-h_{\text {WFSL at }} T_{C}\right)+v_{\text {WFL }}\left(P_{S T M}+P_{N C}\right) \cdot
\end{aligned}
$$

$V_{W F L}\left(P_{S T M}+P_{N C}\right)$ can be neglected relative to the first term, which is larger than the heat of vaporization of the working fluid at the condensing temperature, $h_{f g W F}$ at $T_{C}$.

$$
\begin{aligned}
& \left(h_{\text {WFVout }}-h_{\text {WFLout }}\right)=\left(h_{\text {WFVout }}-h_{\text {WFSL at }} T_{C}\right)+\left(h_{\text {WFSL at }} T_{C}-h_{\text {WFLout }}\right) \\
& =h_{f g W F} \text { at } T_{C}-v_{W F L}\left(P_{S T M}+P_{N C}\right) \\
& \stackrel{\circ}{\mathrm{fgWF}} \text { at } \mathrm{T}_{\mathrm{C}} \text {. }
\end{aligned}
$$

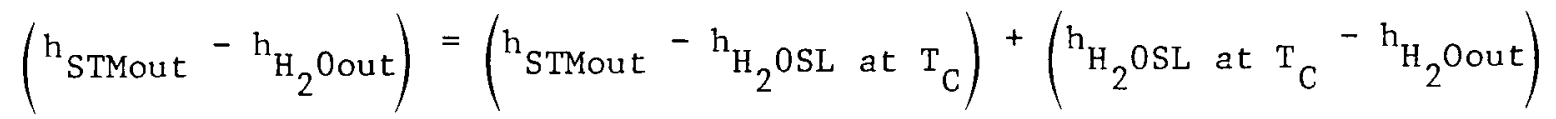

$$
\begin{aligned}
& \doteq \mathrm{h}_{\mathrm{fgH}_{2}} \mathrm{O} \text { at } \mathrm{T}_{\mathrm{C}}+\Delta \mathrm{T}_{\mathrm{PC}} \\
& \frac{\mathrm{W}_{\text {STMin }}}{\mathrm{W}_{\mathrm{H}_{2} \text { Oin }}}=\frac{\mathrm{W}_{\text {STMin }}}{\mathrm{W}_{\text {WFVin }}} \frac{\mathrm{W}_{\text {WFVin }}}{\mathrm{W}_{\mathrm{H}_{2}} \text { Oin }} . \\
& \frac{\mathrm{W}_{\text {WFVout }}}{\mathrm{W}_{\mathrm{H}_{2} \text { Oin }}}=\frac{\mathrm{W}_{\text {WFVout }}}{\mathrm{W}_{\mathrm{NC}}} \frac{\mathrm{W}_{\text {NC }}}{\mathrm{W}_{\text {WFV in }}} \frac{\mathrm{W}_{\text {WFV in }}}{\mathrm{W}_{\mathrm{H}_{2}} \text { Oin }} . \\
& \frac{W_{\text {WFVout }}}{W_{N C}}=\frac{P_{W F}}{P_{N C}} \frac{M_{W F}}{M_{N C}}
\end{aligned}
$$

using the partial pressure ratio method for calculation for vapor flows, and equals $\frac{{ }_{N C} \text { at } T_{C} \text { and } P_{N C}}{{ }_{\text {VWFSV }} \text { at } T_{C}}$ using the specific-volume ratio method. The subscript WFSV denotes working-fluid saturated vapor. 


$$
\frac{\mathrm{W}_{\text {STMout }}}{\mathrm{W}_{\mathrm{H}_{2} \text { Oin }}}=\frac{\mathrm{W}_{\text {STMout }}}{\mathrm{W}_{\mathrm{NC}}} \frac{\mathrm{W}_{\mathrm{NC}}}{\mathrm{W}_{\text {WFVin }}} \frac{\mathrm{W}_{\text {WFVin }}}{\mathrm{W}_{\mathrm{H}_{2}} \text { Oin }}
$$

where

$$
\frac{\mathrm{W}_{\text {STMout }}}{\mathrm{W}_{\mathrm{NC}}}=\frac{\mathrm{P}_{\text {STM }}}{\mathrm{P}_{\mathrm{NC}}} \frac{\mathrm{M}_{\mathrm{STM}}}{{ }_{\mathrm{NC}}} \text { or } \frac{\mathrm{v}_{\mathrm{NC}} \text { at } \mathrm{T}_{\mathrm{C}} \text { and } \mathrm{P}_{\mathrm{NC}}}{{ }_{\text {STM at }} \mathrm{T}_{\mathrm{C}}} \text {. }
$$

Substituting Equations (31) through (42) into Equation (30), the ratio of cooling water to working fluid can be written as:

$$
\begin{aligned}
& \frac{\mathrm{W}_{\mathrm{H}_{2} \text { Oin }}}{\mathrm{W}_{\text {WFVin }}}=\frac{\frac{\mathrm{W}_{\text {STMin }} \mathrm{h}_{\text {STMin }}+\mathrm{W}_{\text {WFVin }} h_{\text {WFVin }}}{\mathrm{W}_{\text {WFVin }}}-\frac{\mathrm{W}_{\text {STMin }}}{\mathrm{W}_{\text {WFVin }}}\left(\mathrm{h}_{\mathrm{H}_{2} \text { OSL at }} \mathrm{T}_{\mathrm{C}}-\Delta \mathrm{T}_{\mathrm{PC}}\right)}{\mathrm{C}_{\mathrm{PH}_{2} \mathrm{O}}\left(\mathrm{T}_{\mathrm{H}_{2} \text { Oout }}-\mathrm{T}_{\mathrm{H}_{2} \text { Oin }}\right. \text { Oin }}
\end{aligned}
$$

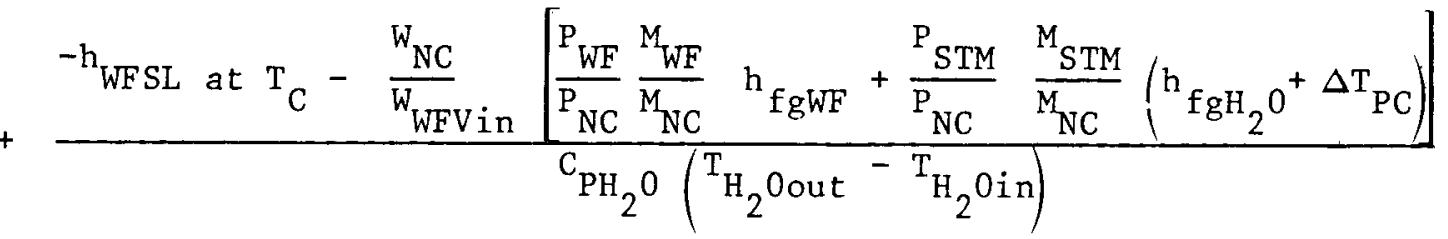

using the partial-pressure ratio method for calculation of vapor flows, and:

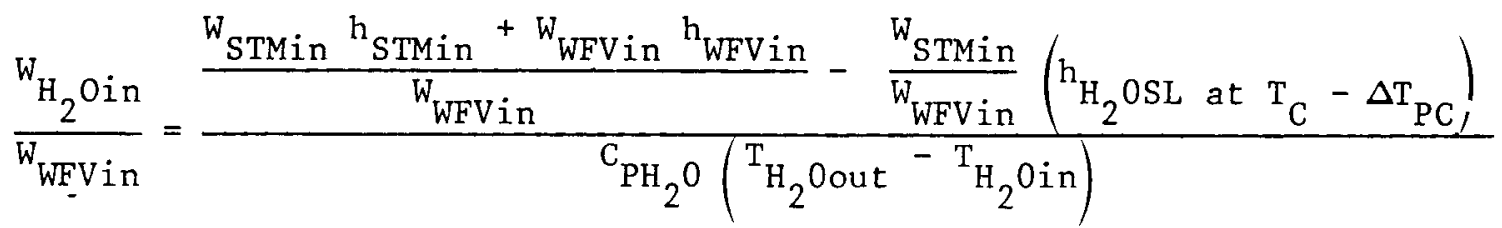

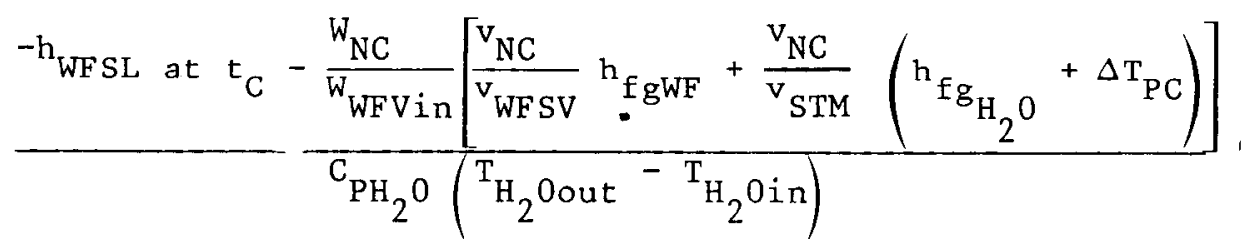

using the specific-volume ratio method.

Equations (43) and (43a) have been grouped such that the first term in the numerator can be calculated knowing the total enthalpies and total flows discharged from the turbines and the postflash vapor compressor, rather than needing enthalpies of the water and working-fluid components separately as they enter the condenser.

Excess water is condensed in the condenser as a result of steam flowing through the turbine along with the working fluid, and steam entering the condenser from the postflash working-fluid recovery system. This excess can be calculated as: 


$$
\frac{\mathrm{W}_{\mathrm{H}_{2} \text { Oout }}-\mathrm{W}_{\mathrm{H}_{2} \text { Oin }}}{\mathrm{W}_{\mathrm{H}_{2} \text { Oin }}}=\frac{\mathrm{W}_{\text {WFVin }}}{\mathrm{W}_{\mathrm{H}_{2} \text { Oin }}}\left(\frac{\mathrm{W}_{\text {STMin }}}{\mathrm{W}_{\text {WFVin }}}-\frac{\mathrm{W}_{\text {STMout }}}{\mathrm{W}_{\mathrm{NC}}} \frac{\mathrm{W}_{\text {NC }}}{\mathrm{W}_{\text {WFVin }}}\right) .
$$

In the solution of the condenser equations, $\mathrm{T}_{\mathrm{C}}$ is selected, and $\mathrm{P}_{\mathrm{NC}}$ is selected. With these quantities selected, and the turbine and postflash vapor compressor discharge conditions known, the terms in Equations (43) or (43a) and (44) can be determined. $\Delta \mathrm{T}_{\mathrm{PC}}$, the condenser pinch point temperature increment, is an empirically selected quantity which depends on the condenser design. $\mathrm{T}_{\mathrm{H}_{2}}$ Oout $-\mathrm{T}_{\mathrm{H}_{2}} \mathrm{Oin}$ (plus the cooling-water temperature increment resulting from condensing the vent vapors from the primary condenser) is equal to the cooling-water temperature drop provided by the cooling tower.

\subsection{Effects of Noncondensible Solubility}

Equations (43) and (43a) have been derived with the assumption that all the noncondensibles entering the condenser leave with the vent vapors, and do not dissolve in either working fluid or water. A more complete treatment of noncondensible solubility would require accounting for the noncondensibles leaving the condenser with liquid flows (water and working fluid) for the selected total condenser pressure, by using the appropriate Henry's constants. This was not done in the present hand calculations.

Nitrogen, the primary noncondensible considered for Raft River conditions, has a small solubility in working fluid and a very small solubility in water. Therefore, a fraction of the noncondensibles entering the condenser will leave in the condensate stream. However, for the working-fluid recovery systems evaluated numerically in this report, the only significant path of exit from the system for the noncondensibles is through the condenser vent. Noncondensibles which leave the condenser dissolved in working fluid will be recycled through the boilers and turbines, and will reenter the condenser. Any noncondensible trying to leave the plant with spent geo-fluid will, unfortunately, be recovered along with the working-fluid vapor in the postflash, and returned to the condenser. Therefore, the amounts of noncondensible, water vapor, and working-fluid vapor vented from the condenser, for a given noncondensible partial pressure in the condenser, should be quite accurately estimated by Equations (43) or (43a). It follows then, that although the condensate will contain a small concentration of dissolved noncondensible which is not specifically accounted for in the equations, the amount of working fluid and water vapors condensed, and the corresponding cooling water required, were predicted approximately correctly by Equations (43) and (43a).

\subsection{Condensing of Mixed-Hydrocarbon Working Fluids}

A significant difference exists between the condensation process for pure and mixed-hydrocarbon working fluids. The mixed working fluids condense over a range of temperatures at a constant condensing pressure. A proper condenser, therefore, must provide countercurrent-flow heat exchange between the cooling water and the working fluid. The pinch point, or minimum temperature difference between the working fluid and cooling water, may occur at either the cooling-water inlet or outlet, or at some intermediate location depending on the cooling-water temperature rise, the amount of desuperheat required, and the temperature range over which condensation occurs. Cooling water required for condensation can be calculated in much the same way as for a pure fluid; however, it is convenient to use the direct-contact condenser balance equation in a rewritten form. For this application Equation (43a) can be written as: 


$$
\begin{aligned}
& \frac{W_{H_{2} \text { Oin }}}{{ }_{\text {WFVin }}}=\frac{\frac{W_{\text {STMin }} h_{\text {STMin }}+W_{\text {WFVin }} h_{\text {WFVin }}}{W_{\text {WFVin }}}-\frac{W_{\text {STMin }}}{W_{\text {WFVin }}}\left(h_{H_{2} \text { Oout }}\right)-h_{\text {WFLout }}}{C_{\mathrm{PH}_{2} O}\left({ }_{\mathrm{H}_{2} \text { Oout }}-\mathrm{T}_{\mathrm{H}_{2} \text { Oin }}\right)} \\
& -\frac{\left(\frac{\mathrm{W}_{\mathrm{NC}}}{\mathrm{W}_{\text {WFVin }}}\right)\left(\frac{\mathrm{v}_{\mathrm{NC}}}{\mathrm{v}_{\text {WFSV }}}\right)\left(\mathrm{h}_{\text {WFSV at }} \mathrm{T}_{\mathrm{C}}-\mathrm{h}_{\text {WFSL at }} \mathrm{T}_{\mathrm{C}}\right)}{\mathrm{CH}_{2} \mathrm{O}\left(\mathrm{T}_{\mathrm{H}_{2} \text { Oout }}-\mathrm{T}_{\mathrm{H}_{2}} \text { Oin }\right)} \\
& -\frac{\left(\frac{\mathrm{W}_{\mathrm{NC}}}{\mathrm{W}_{\text {WFVin }}}\right)\left(\frac{\mathrm{v}_{\mathrm{NC}}}{\mathrm{v}_{\text {STMout }}}\right)\left(\mathrm{h}_{\mathrm{H}_{2} \text { OSV at }} \mathrm{T}_{\mathrm{C}}-\mathrm{h}_{\mathrm{H}_{2} \text { Oout }}\right)}{\mathrm{C}_{\mathrm{PH}_{2} \mathrm{O}\left(\mathrm{T}_{2}\right. \text { Oout }}-\mathrm{T}_{\left.\mathrm{H}_{2} \text { Oin }\right)}} .
\end{aligned}
$$

For mixed fluids, $\mathrm{T}_{\mathrm{C}}$ in the above equation is the temperature at which condensing is complete (bubble point temperature). Proper application of this equation requires that the condenser inlet and outlet conditions are consistant with an appropriate condenser pinch point.

\section{STEADY-FLOW, EQUILIBRIUM FLASHING}

\subsection{Mass and Energy Balances}

Equations suitable for the calculation of steady-flow equilibrium flashing of a solvent-solute fluid were constructed using, basically, mass and energy balances, and supplementary relations based on Henry's law for determining the amount of vapor dissolved in geo-fluid. As for boiler and condenser analyses, two approaches for calculation of mixtures of vapor flows can be applied: the partial-pressure ratio, and the specific-volume ratio methods. Both approaches are treated in this section, although the partial-pressure ratio approach is considerably easier to apply for hand calculations, and was used in the reported analyses.

It is recognized that in an actual flashing process, complete equilibrium will not be achieved. The assumption made in the application of the equilibrium flashing equations is that the calculated power penalties will represent first approximations, and that a more refined treatment must await experimental data from appropriate flash equipment.

Referring to the flash-tank schematic in Figure 6, the mass balance can be written as:

$$
\begin{aligned}
& w_{\text {GFin }}=w_{\text {GFout }}+w_{\text {STM }} \\
& w_{\text {Sin }}=w_{\text {Sout }}+w_{\text {SVout }}
\end{aligned}
$$

where the subscript S refers to the solute dissolved in the geo-fluid, and SV refers to the solute which has come out of solution to the vapor state. (The other notation has been previously defined.) 
The energy balance can be written:

$$
\begin{aligned}
& w_{\text {GFin }}{ }^{h} \text { GFin }+w_{\text {Sin }}{ }^{h} \text { Sin } \\
&=w_{\text {STM }} h_{\text {STM }}+w_{\text {SVout }}{ }^{h} \text { SVout } \\
&+w_{\text {GFout }}{ }^{h} \text { GFout } \\
&+w_{\text {Sout }}{ }^{h} \text { Sout } \\
&
\end{aligned}
$$

The mass and energy balances can be combined to give:

$$
\begin{gathered}
\left(h_{\text {GFin }}-h_{\text {GFout }}\right)+\frac{\dot{W}_{\text {Sout }}}{W_{\text {GFin }}}\left(h_{\text {Sin }}-h_{\text {Sout }}\right)+\frac{W_{\text {SVout }}}{W_{\text {GFin }}}\left(h_{\text {Sin }}-h_{\text {SVout }}\right) \\
=\frac{W_{\text {STM }}}{W_{\text {GFin }}}\left(h_{\text {STM }}-h_{\text {GFout }}\right) .
\end{gathered}
$$

The terms in Equation (49) can be rewritten as follows:

$$
\begin{aligned}
\left(\mathrm{h}_{\text {GFin }}-\mathrm{h}_{\text {GFout }}\right) & =\left(\mathrm{h}_{\text {GFin }}-\mathrm{h}_{\text {GFSL at }} \mathrm{T}_{\text {FLASH }}\right)+\left(\mathrm{h}_{\text {GFSL at }} \mathrm{T}_{\text {FLASH }}-\mathrm{h}_{\text {GFout }}\right) \\
& =\left(\mathrm{h}_{\text {GFin }}-\mathrm{h}_{\text {GFSL at }} \mathrm{T}_{\text {FLASH }}\right)-\mathrm{v}_{\text {GFSL }} \mathrm{P}_{\text {SV }}
\end{aligned}
$$

where

$v_{\text {GFSL }}$ is the specific volume for the saturated liquid geo-fluid or brine at the flash temperature.

The term ${ }_{G}$ GFSL ${ }^{P}$ SV is generally small relative to $\left(h_{G F i n}-h_{G F S L}\right.$ at $\left.T_{F L A S H}\right)$, but has been included in the flash equations for completeness.

$$
\frac{\mathrm{W}_{\text {Sout }}}{\mathrm{W}_{\text {GFin }}}\left(\mathrm{h}_{\text {Sin }}-\mathrm{h}_{\text {Sout }}\right) \doteq \frac{\mathrm{C}_{\text {PS }}}{\mathrm{C}_{\text {PGF }}} \frac{\mathrm{W}_{\text {Sout }}}{\mathrm{W}_{\text {GFin }}}\left(\mathrm{h}_{\text {GFin }}-\mathrm{h}_{\text {GFout }}\right),
$$

because the solute and geo-fluid have the same temperature at a given location. In the above equation, $\mathrm{C}_{\mathrm{PS}}$ and $\mathrm{C}_{\mathrm{PGF}}$ are the specific heats of the liquid solute and geo-fluid.

Since $\frac{C_{P S}}{C_{P G F}} \frac{W_{\text {Sout }}}{W_{\text {GFin }}}$ is much less than unity for the present application, the second term in Equation (49) can be neglected relative to the first.

$$
\begin{aligned}
\left(h_{\text {Sin }}-h_{\text {SVout }}\right) & =\left(h_{\text {Sin }}-h_{\text {Sout }}\right)+\left(h_{\text {Sout }}-h_{\text {SVout }}\right) \\
& =\left(h_{\text {Sin }}-h_{\text {Sout }}\right)+\Delta h_{\text {SOLN }}
\end{aligned}
$$


where $\Delta \mathrm{h}_{\text {SOLN }}$ represents the change in enthalpy associated with the solute coming out of solution at the flash temperature. Then the third term in Equation (49) becomes:

$$
\frac{\mathrm{w}_{\text {SVout }}}{\mathrm{W}_{\text {GFin }}}\left(\mathrm{h}_{\text {Sin }}-\mathrm{h}_{\text {SVout }}\right)=\frac{\mathrm{C}_{\text {PS }}}{\mathrm{C}_{\text {PGF }}} \frac{\mathrm{W}_{\text {SVout }}}{\mathrm{W}_{\text {GFin }}}\left(\mathrm{h}_{\text {GFin }}-\mathrm{h}_{\text {GFout }}\right)+\frac{\mathrm{W}_{\text {SVout }}}{\mathrm{W}_{\text {GFin }}} \Delta \mathrm{h}_{\text {SOLN }}
$$

Since

$$
\begin{aligned}
\frac{\mathrm{C}_{\text {PS }}}{\mathrm{C}_{\text {PGF }}} \frac{\mathrm{W}_{\text {SVout }}}{\mathrm{W}_{\text {GFin }}} & \ll 1.0 \text {, the third term in Equation (49) can be written: } \\
\frac{\mathrm{W}_{\text {SVout }}}{\mathrm{W}_{\text {GFin }}}\left(\mathrm{h}_{\text {Sin }}-\mathrm{h}_{\text {SVout }}\right) \doteq \frac{\mathrm{W}_{\text {SVout }}}{\mathrm{W}_{\text {STM }}} \frac{\mathrm{W}_{\text {STM }}}{\mathrm{W}_{\text {GF in }}} \Delta \mathrm{h}_{\text {SOLN }} & \\
\left(\mathrm{h}_{\text {STM }}-\mathrm{h}_{\text {GFout }}\right) & =\left(\mathrm{h}_{\text {STM }}-\mathrm{h}_{\text {GFSL at }} \mathrm{T}_{\text {FLASH }}\right)+\left(\mathrm{h}_{\text {GFSL at }} \mathrm{T}_{\text {FLASH }}-\mathrm{h}_{\text {GFout }}\right) \\
& \doteq \mathrm{h}_{\text {fg GF }} \text { at } \mathrm{T}_{\text {FLASH }}
\end{aligned}
$$

since

$$
\left(h_{\text {GFSL at }} \mathrm{T}_{\text {FLASH }}-\mathrm{h}_{\text {GFout }}\right)=\mathrm{v}_{\text {GFSL }}{ }^{\mathrm{P}}{ }_{\mathrm{SV}} \ll \mathrm{h}_{\mathrm{fgGF}} \cdot
$$

The energy balance can be written by substituting Equations (50), (54), and (55) into Equation (49).

$$
\frac{W_{\text {STM }}}{W_{\text {GFin }}}=\frac{\left(h_{\text {GFin }}-h_{\text {GFSL at }} T_{\text {FLASH }}\right)-v_{\text {GFSL }} P_{\text {SV }}}{h_{\text {fgGF }} \text { at } T_{\text {FLASH }}\left(1-\frac{\mathrm{W}_{\text {SVout }}}{W_{\text {STM }}} \frac{\Delta h_{\text {SOLN }}}{h_{\text {fgGF }} \text { at } T_{\text {FLASH }}}\right)} \text {. }
$$

Recall that $\mathrm{P}_{\mathrm{SV}}$ is the solute vapor pressure in the flash tank, and is mainly a function of solute concentration rather than $\mathrm{T}_{\text {FLASH }}$. To calculate $\mathrm{P}_{\mathrm{SV}}$ and the total flash pressure, the expression

$$
P_{\text {FLASH }}=P_{S V}+P_{S T M} \text { at } T_{\text {FLASH }} \text {, }
$$

the continuity relationships, and Henry's law are utilized.

From continuity:

$$
\frac{W_{\text {SVout }}}{W_{\text {STM }}}+\frac{W_{\text {Sout }}}{W_{\text {GFout }}}\left(\frac{W_{\text {GEin }}}{W_{\text {STM }}}-1\right)=\frac{W_{\text {Sin }}}{W_{\text {GFin }}} \frac{W_{\text {GFin }}}{W_{\text {STM }}} .
$$

Equation (58) can be rearranged to give: 


$$
\frac{\mathrm{W}_{\text {STM }}}{\mathrm{W}_{\text {GFin }}}=\frac{\frac{\mathrm{w}_{\text {Sin }}}{\mathrm{W}_{\text {GFin }}}-\frac{\mathrm{w}_{\text {Sout }}}{\mathrm{W}_{\text {GFout }}}}{\frac{\mathrm{W}_{\text {SVout }}}{\mathrm{W}_{\text {STM }}}-\frac{\mathrm{w}_{\text {Sout }}}{\mathrm{W}_{\text {GF out }}}}
$$

where $\frac{W_{\text {Sin }}}{W_{\text {GFin }}}$ is assumed known.

$$
\frac{\mathrm{W}_{\text {Sout }}}{\mathrm{W}_{\text {GFout }}}=\mathrm{H}^{\prime} \quad \mathrm{P}_{\mathrm{SV}} \text {. }
$$

Where $\mathrm{H}^{\prime}$ is a modified Henry constant for the solute and equals $\frac{\text { mass fraction of solute in solution }}{\text { partial pressure of solute vapor (psia) }}$.

$$
\frac{\mathrm{W}_{\text {SVout }}}{\mathrm{W}_{\text {STM }}}=\frac{\mathrm{P}_{\text {SV }}}{\mathrm{P}_{\text {STM }} \text { at } \mathrm{T}_{\text {FLASH }}} \frac{\mathrm{M}_{S}}{\mathrm{M}_{\text {STM }}} \text { or } \frac{\mathrm{v}_{\text {STM at }} \mathrm{T}_{\text {FLASH }}}{\mathrm{v}_{\text {SV at } T_{\text {FLASH }}, \mathrm{P}_{\text {SV }}}}
$$

assuming the partial-pressure ratio method of calculating vapor flows or the specific-volume ratio, respectively, is applicable. Equation (59) becomes:

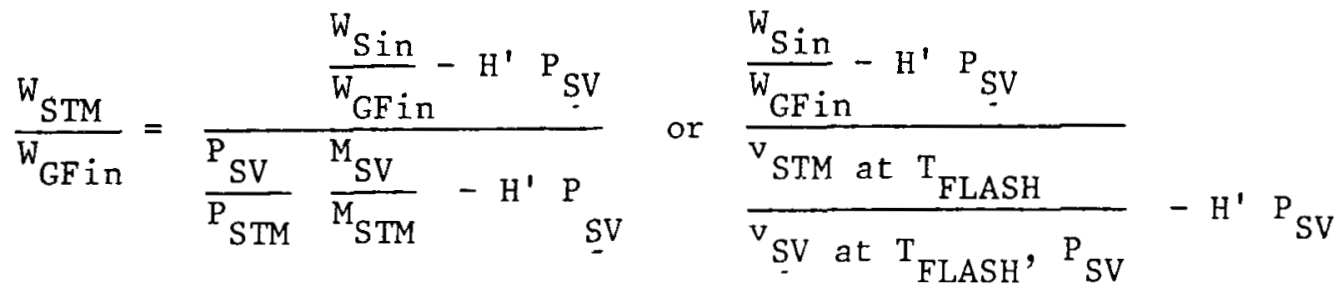

for the partial-pressure ratio or for the specific-volume ratio method.

In the general case, using either approach for calculating vapor flows, Equations (56), (61), and (62) can

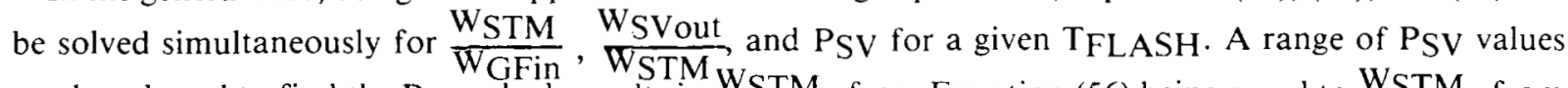
can be selected to find the PSV which results in $\frac{W_{S T M}}{W_{\text {GFin }}}$ from Equation (56) being equal to $\frac{\text { W }_{\text {STM }}}{\text { W }_{\text {GFin }}}$ from Equation (62).

\subsection{Simplified Flash Equations}

A simpler solution to these equations, not requiring iteration, exists if the method of partial-pressure ratios is used to calculate vapor flows, and if the terms $v_{\text {GFSL }} P_{S V}$ and $\frac{\Delta h_{S O L N}}{h_{f g G F} \text { at } T_{\text {FLASH }}}$ in Equation (56) are neglected. (Several sample calculations were conducted representing the postflash conditions for the spent geo-fluid to assess the effects of neglecting the two terms. The conclusion was reached that for the present application of these equations, the terms could safely be neglected.)

With these simplifications, Equation (56) becomes: 


$$
\frac{W_{\text {STM }}}{W_{\text {GFin }}}=\frac{h_{\text {GFin }}-h_{\text {GFSL at }} T_{\text {FLASH }}}{h_{\text {fgGF at }} T_{\text {FLASH }}}
$$

and from Equation (62):

$$
\mathrm{P}_{S V}=\frac{\frac{\mathrm{W}_{\text {Sin }}}{\mathrm{W}_{\text {GFin }}}}{\frac{1}{\mathrm{P}_{\text {STM }} \text { at } \mathrm{T}_{\text {FLASH }}} \frac{\mathrm{M}_{\mathrm{S}}}{\mathrm{M}_{\text {STM }}} \frac{\mathrm{W}_{\text {STM }}}{\mathrm{W}_{\text {GF in }}}+\mathrm{H}^{\prime}\left(1-\frac{\mathrm{W}_{\text {STM }}}{\mathrm{W}_{\text {GFin }}}\right)} \cdot
$$

Equations (63) and (64) were used for the calculations presented in this report.

\subsection{Flashing with Several Dissolved Gases}

Using the simplified equations, calculations were made for flashing the cooling water upstream of a wet cooling tower to recover working fluid. For that condition the cooling water will contain dissolved nitrogen and oxygen, and those gases must be accounted for in the calculation. For this purpose mass balances can be written for each vapor separately, and solutions similar to Equations (63) and (64) obtained. The solution becomes:

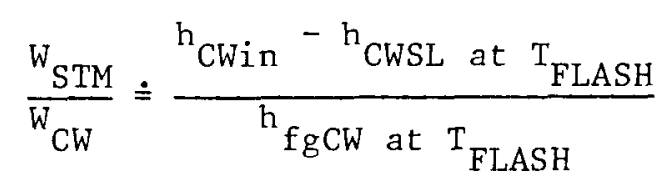

and

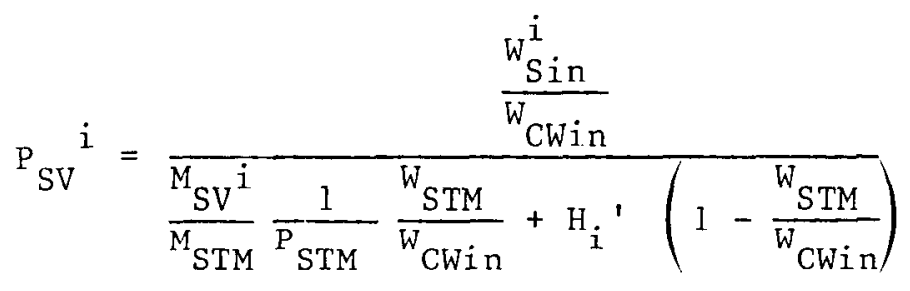

where $\mathrm{CW}$ denotes cooling water, and i refers to any of the dissolved gases.

A solution procedure is to select $\mathrm{T}_{\text {FLASH }}$, solve for $\frac{\mathrm{w}_{\mathrm{STM}}}{\mathrm{W}_{\mathrm{CW}}}$ using Equation (65), and then solve for the individual partial pressures of the dissolved gases using Equation (66). Finally:

$$
\mathrm{P}_{\mathrm{FLASH}}=\mathrm{P}_{\mathrm{STM}}+\sum_{\mathrm{i}} \mathrm{P}_{\mathrm{SV}}^{\mathrm{i}} \text {. }
$$

\section{REFRIGERATION CALCULATIONS}

One approach assessed for processing the condenser-vent vapor to recover working fluid which is vented with the noncondensibles, consists of refrigeration of those vapors. At the reduced temperature, condensation of working fluid and water vapor will occur, and the resulting vapor will have a reduced mass fraction of working fluid to noncondensibles. Accordingly, as the fixed flow of noncondensibles is subsequently vented, a reduced amount of working fluid will be lost to the atmosphere. The major parasitic power 
required for the process consists of the change in enthalpy of the condenser-vent flow during its refrigeration divided by the coefficient of performance, $\frac{Q}{W}$, for the refrigeration cycle. The work required to pump the condensate back to the inlet of the working-fluid feed pump can be neglected.

The basic refrigeration cycle assumed is shown in a P-h diagram below.

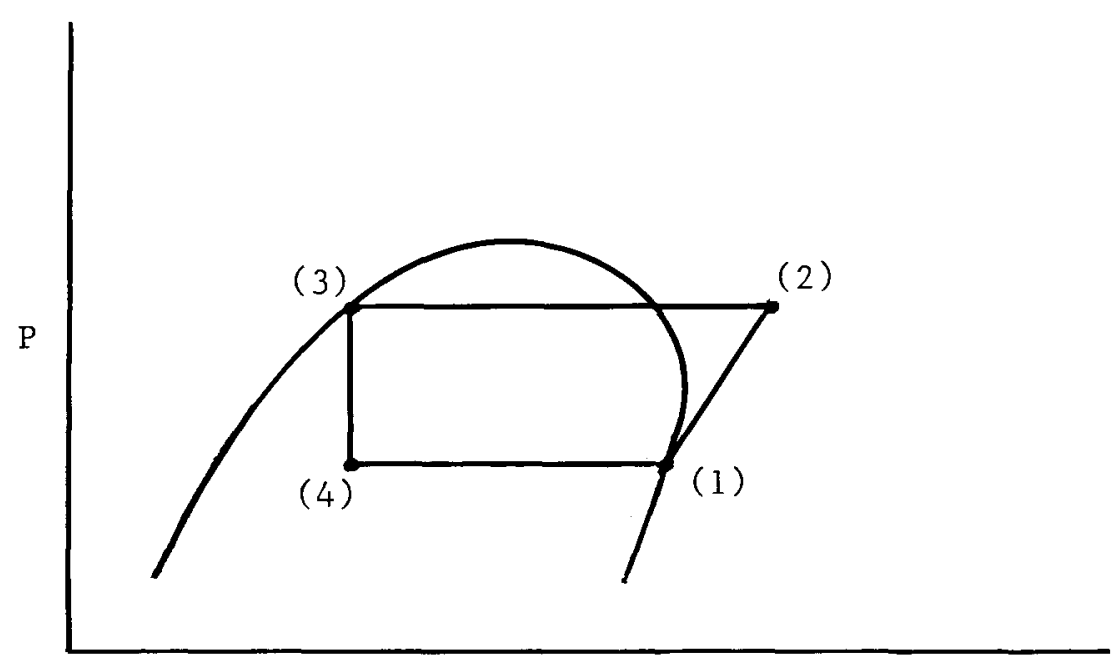

h

At state point 1 in the diagram the refrigerant has just finished absorbing heat from the condenser-vent products and is ready to be compressed. An approach temperature difference of $10^{\circ} \mathrm{F}$ was assumed between the refrigerant and the condenser-vent vapors at this point; the refrigerant is $10^{\circ} \mathrm{F}$ colder than the vent-product condensing temperature, which is selected as a parameter. The heat removed from the condenser-vent vapors corresponds to $\left(\mathrm{h}_{1}-\mathrm{h}_{4}\right)$.

At state point 2 in the diagram, the refrigerant has just been compressed (with a compression efficiency assumed equal to 0.80 ) to the refrigerant condensing pressure. The compression work is $\mathrm{W}=\mathrm{h}_{2}-\mathrm{h}_{1}$. The refrigerant condensing pressure corresponds to the pressure of saturated refrigerant vapor at the refrigerant condensing temperature. An approach temperature difference of $10^{\circ} \mathrm{F}$ was assumed between the refrigerant condensing temperature and the heat sink temperature of the cooling water. The ratio of heat removed to work required, $\frac{h_{1}-h_{4}}{h_{2}-h_{1}}$, is the coefficient of performance.

At state point 3 in the diagram, the refrigerant has been desuperheated and condensed at its condensing temperature. The heat rejected to the cooling water is $h_{2}-h_{3}$.

At state point 4 in the diagram, the refrigerant has been cooled by expansion through a throttling valve $\left(h_{4}=h_{3}\right)$, from the refrigerant condensing pressure to the vapor pressure which corresponds to the refrigeration cycle cold temperature (vent-product condensing temperature $-10^{\circ} \mathrm{F}$ ).

For each geothermal cycle point assessed, the condenser-vent product condensing temperature was selected, which determines a refrigeration cycle cold temperature. A constant refrigeration cycle hot temperature of $105^{\circ} \mathrm{F}$ was assumed, corresponding to a cooling-water temperature of $95^{\circ} \mathrm{F}$. The enthalpy change of the vent products was calculated for the vent-vapor condensation process, and finally the refrigeration power calculated for that point. 


\section{ANALYSES}

\section{SCOPE OF ANALYSES}

Cycle state points and power balances have been calculated using the analysis methods discussed in Section III. The purpose was to examine the behavior of both shell-and-tube and direct-contact binary cycles, and to investigate methods for reducing working-fluid loss rates to acceptable levels for the directcontact cycles. Results of these analyses are presented in Sections IV-2, IV-3, IV-4, and IV-6. A preliminary assessment of heat rejection systems is discussed in Section IV-5.

\section{CYCLE SCREENING}

\subsection{Background}

This section includes the results of a number of geothermal binary-cycle calculations made with the objective of finding a cycle which can produce low-cost electrical energy from a moderately lowtemperature geothermal resource. Raft River geo-fluid with a temperature of $290^{\circ} \mathrm{F}$ and 52 parts per million dissolved nitrogen was assumed for all analyses.

Several cycles were screened which included isobutane, pentane, cis-2-butene, and several mixedhydrocarbon working fluids. Dual- and triple-boiling cycles were analyzed. Both shell-and-tube and directcontact boilers, heaters, and condensers were assessed. Wet cooling towers were assumed. For the cycle screening effort, working-fluid recovery parasitic losses were not assessed.

The current level of understanding of cooling-tower state of the art at EG\&G Idaho, Inc., suggests that a significant parasitic penalty to the cycle, as well as relatively large equipment costs, would accompany the use of any tower other than a wet tower. The use of other types is being investigated, but the present analyses assumed wet cooling towers throughout.

The cycles analyzed were configured so that the geo-fluid passes in series through the high-pressure boiler, high-pressure heater, medium- or low-pressure boiler, medium- or low-pressure heater, etc. For triple-boiling cycles, for example, the entire flow of working fluid initially passes through the low-pressure heater, then splits between the low-pressure boiler and medium-pressure heater. The working-fluid flow splits again after leaving the medium-pressure heater, flowing in part into the medium-pressure boiler, and in part into the high-pressure heater. Dual-boiling cycles were arranged comparably. Figures 1 and 5 illustrate these dual- and triple-boiling cycle configurations.

Boehm and Jacobs, at the University of Utah, have investigated split-brine flow, dual-boiling cycles for several working fluids. In that type of cycle the geothermal fluid (or brine) first flows through the highpressure boiler, and then is split. Just sufficient geo-fluid is passed through the high-pressure heater to maintain an approximately constant temperature difference between the geothermal and working fluids, the remainder of the geo-fluid flowing in series through the low-pressure boiler and preheater. The University of Utah calculations have considered both isobutane and pentane for the high-pressure working fluid, and have included several additional working fluids for the low-pressure part of the cycle. Two conclusions were reached upon reviewing those results:

(1) For isobutane, the split-brine flow cycle appears to produce essentially the same net plant performance as the corresponding cycle shown in Figure 1 of this report (within about $2 \%$ ); for pentane the split-flow cycle showed about $10 \%$ lower performance.

(2) If two working fluids were used in a direct-contact cycle, the problem of separating them from each other after their recovery from the spent geo-fluid would constitute a significant complication to the cycle. 
Accordingly, the decision was made, for the present study, not to include split-brine flow cycles, or cycles which use two or more working fluids. Small improvements in net geo-fluid effectiveness may be achievable through incorporation of the split-brine flow or dual working-fluid features, however, and additional evaluation of these features should be completed before a final selection of the cycle for the second 5-MW plant at Raft River is made.

\subsection{Assumptions}

Several assumptions were made to provide input to the present calculations. Some of these assumptions are in the nature of ground rules, and some were made in lieu of substantial cycle optimization where engineering judgements and/or brief numerical checks suggested that these assumptions do not invalidate the overall conclusions reached. The assumptions are as follows:

(1) The calculations assume that geo-fluid is delivered from the field to the plant at a given temperature and pressure, and that it must be returned to the field at that same pressure. Pumping power was charged to the plant for pumping the geo-fluid to the boiler pressure, for example, and for pumping the geo-fluid leaving the plant to the plant inlet pressure. If a particular cycle resulted in a plant discharge of geo-fluid at a higher pressure than that delivered to the plant, credit was given to the plant for the amount of pump work (at $80 \%$ efficiency) corresponding to the excess discharge pressure.

(2) Reference 1 contains calculations for dual-boiling, shell-and-tube cycles for several working fluids, which permitted selecting optimum boiler temperatures for a given working-fluid and geo-fluid temperature. Boiler temperatures for the present calculations were taken from those optima and assumed to represent near-optimum temperatures for these direct-contact cycles. A partial check of this assumption will be discussed in Section IV-6.

(3) For both direct-contact and shell-and-tube boilers/heaters the working fluid was assumed to be vaporized with zero superheat, and to enter the boiler at the boiling temperature and pressure. For all the analyses, working fluid carryunder was assumed negligible.

(4) Pump efficiencies were assumed to be $80 \%$. Turbine efficiencies were assumed to be $85 \%$. The extremely small fraction of water moisture calculated for an isentropic process through the turbine was assumed not to invalidate this assumption. Electrical losses were not considered.

(5) Component and piping frictional pressure drops were neglected, as was power required to provide small boosts in pressure between corresponding heaters and boilers.

(6) The cooling-water temperature drop was assumed to be about $20^{\circ} \mathrm{F}$ in the cooling tower, with the condenser inlet temperature equal to $75^{\circ} \mathrm{F}$. Assessments of cooling-tower fan power and cooling-water pumping power have not been made in detail for the present calculations. Basic wet-tower power requirements in watts were taken from Reference 1 as a function of cooling-water flow, and are equal to 0.077 times the cooling-water flow in $\mathrm{lbm} / \mathrm{hr}$. This parasitic loss was applied directly for shell-and-tube condensers. For directcontact condensers an extra parasitic loss was included as the power required to pump the cooling water from the cooling tower to the condenser pressure.

(7) Water properties were taken from ASME Steam Tables (Reference 3), and pentane and isobutane properties as published by K. Starling (Reference 4 ) were used. The computer code THERPP 9 was used to calculate properties of the mixed-hydrocarbon working fluids. 


\subsection{Cycle Screening Results}

2.3.1 General. The results of the cycle screening study are presented in Tables I and II. For each of the cases analyzed, the cycle is defined by the boiler temperatures, the condenser temperatures, and the working fluid. Included in the power balance are total turbine power, geo-fluid pumping power, workingfluid pumping power, and cooling-tower power. Working-fluid recovery parasitics have not been included. Net power has been estimated for both direct-contact and shell-and-tube condensers. All of the power balance numbers shown have units of watt-hr/lbm geo-fluid.

2.3.2 Case Descriptions. The first twelve cases are for cycles having direct-contact boilers and heaters (Table I); the last four, for shell-and-tube cycles (Table II). Boiler/heater pinch points were taken as $10^{\circ} \mathrm{F}$. Condehser pinch points were varied to some extent and are specified in the short description of each case following.

Case 1. This case is a dual-boiling, direct-contact isobutane cycle with a single condenser. The boiler temperatures are 240 and $180^{\circ} \mathrm{F}$. The condensing temperature is $104^{\circ} \mathrm{F}$ which corresponds to a $9^{\circ} \mathrm{F}$ pinch point in the condenser. The nitrogen partial pressure in the condenser was taken as 2.2 psia.

Case 2. The boiler temperatures for this triple-boiling, triple-condensing, direct-contact isobutane cycle are 260,210 , and $150^{\circ} \mathrm{F}$. Three condensers were connected in series to the cooling water. The condensing temperatures for the high-, medium-, and low-pressure turbines were 91,99 , and $104^{\circ} \mathrm{F}$, respectively. These temperatures correspond to $9^{\circ} \mathrm{F}$ condenser pinch points. Although not shown in Table I, workingfluid recovery losses were calculated for this cycle, and are described in Section IV-3.2. The cycle includes a geothermal-fluid preflash which lowered the nitrogen partial pressure in the condenser to $1.0 \mathrm{psia}$ and increased the geo-fluid delivery pumping power to 0.52 watt-hr/lbm geo-fluid.

Case 3. This case is for a dual-boiling pentane cycle. The single-condensing temperature is $104^{\circ} \mathrm{F}$ with a pinch point of $9^{\circ} \mathrm{F}$. The nitrogen partial pressure in the condenser was assumed to be 0.35 psia.

Case 4. This case is the same as Case 3 except for the use of two condensers having condensing temperatures of $94.9^{\circ} \mathrm{F}$ for the high-pressure turbine and $104^{\circ} \mathrm{F}$ for the low-pressure turbine. Condenser pinch points are both $9^{\circ} \mathrm{F}$.

Case 5. This is a triple-boiling, single-condensing pentane cycle. The condenser has a $9^{\circ} \mathrm{F}$ pinch point, and 0.35 psia nitrogen partial pressure.

Case 6. This is the same as Case 5 except for the use of three condensers with cooling water passing in series through the condensers, starting with the high-pressure turbine condenser. Condenser pinch points are $9^{\circ} \mathrm{F}$, and the nitrogen partial pressure is 0.35 psia in each condenser.

Case 7. This case is a dual-boiling, cis-2-butene cycle having a single condenser. The condenser pinch point is $9^{\circ} \mathrm{F}$ and the nitrogen partial pressure was assumed to be 0.35 psia.

Case 8. This case is dual-boiling, mixed working-fluid cycle using 50\% isobutane and $50 \%$ isopentane to feed the low-pressure heater. The working-fluid composition entering the high-pressure heater is $40 \%$ isobutane and $60 \%$ isopentane. The boiler dew-point temperatures (boiling completed) are 245 and $180^{\circ} \mathrm{F}$. The direct-contact condenser is assumed to be a packed-bed configuration. The condenser bubble point (at which point everything condensible is condensed) was assumed to equal the cooling-water temperature leaving the condenser, which was taken as $95^{\circ} \mathrm{F}$. The nitrogen partial pressure was assumed to equal $1.0 \mathrm{psia}$. For this case the condenser pinch point located at the water inlet, was equal to $95-75=20^{\circ} \mathrm{F}$.

Case 9. This is the same as Case 8 except for the use of dual condensers with the cooling water connected in parallel. For mixed working fluids having condensing temperature increments larger than the $20^{\circ} \mathrm{F}$ cooling-water temperature rise, condenser pinch points occur at the water inlet; hence the multiple 
condensers were connected in parallel. For this case the condenser bubble points were both $95^{\circ} \mathrm{F}$, corresponding to pinch points of $20^{\circ} \mathrm{F}$. The nitrogen partial pressure in the condenser was assumed to equal 1.0 psia.

Case 10. This is the same as Case 9 except for the assumption of $9^{\circ} \mathrm{F}$ condenser pinch points rather than $20^{\circ} \mathrm{F}$ pinch points. The nitrogen partial pressure was assumed to equal $1.0 \mathrm{psia}$. For this assumption a direct-contact condenser must be completely counter flow, so that a single-stage, packed-bed configuration could not be used. A collapsing bubble configuration was assumed with an additional penalty to the turbine exhaust pressure of 1.0 psia (corresponding to about a $2-\mathrm{ft}$ height of the condensing column). The condenser bubble points of $84^{\circ} \mathrm{F}$ correspond to the cooling-water inlet temperature of $75^{\circ} \mathrm{F}$ plus the $9^{\circ} \mathrm{F}$ pinch points.

Case 11. This mixed fluid case used $50 \%$ isobutane and $50 \%$ hexane feeding the low-pressure boiler. Boiling occurs over about a $40^{\circ} \mathrm{F}$ temperature range in the high-pressure boiler. As for Case 10 , two parallel, collapsing bubble type condensers were used with condenser bubble points of $84^{\circ} \mathrm{F}$, and pinch points of $9^{\circ} \mathrm{F}$. A nitrogen partial pressure of 1.0 psia was assumed.

Case 12. This is a dual-boiling, single-condensing cycle using primarily isobutane, but with just sufficient hexane to achieve a $20^{\circ} \mathrm{F}$ condensing temperature increment. The condenser pinch point is $9{ }^{\circ} \mathrm{F}$. A collapsing bubble type of condenser with 1.0 psia nitrogen partial pressure was assumed.

Case 13. This is a reference shell-and-tube, dual-boiling isobutane cycle corresponding to the first Raft River 5-MW Pilot Plant. A $9^{\circ} \mathrm{F}$ pinch point was assumed for the condenser. The geo-fluid delivery pumping power corresponds to pumping the geothermal fluid from 100 to 150 psia as is specified for the first 5-MW Raft River Pilot Plant. Direct-contact condensers were not considered. When this cycle is compared with the corresponding direct-contact cycle with working-fluid recovery parasitics accounted for (see Section IV-6.3), credit should be given for the geo-fluid discharge pressure of 150 psia. This value is $50 \mathrm{psia}$ in excess of the $100 \mathrm{psi}$ discharge pressure assumed for the comparable direct-contact cycle. The comparable net cycle power is $4.06+0.06=4.12$-watt-hr $/ \mathrm{lbm}$ geo-fluid for the reference shell-and-tube cycle.

Case 14. This is a reference shell-and-tube, triple-boiling, triple-condensing, isobutane cycle. The condenser pinch points are $9^{\circ} \mathrm{F}$. When this cycle is compared with the corresponding direct-contact cycle with working-fluid parasites accounted for, the comparable net cycle power is $4.74+0.06=4.80$-watt-hr $/ \mathrm{lbm}$ geo-fluid.

Case 15. This is a reference shell-and-tube, dual-boiling, pentane cycle. The condenser pinch point is $9^{\circ} \mathrm{F}$.

Case 16. This case represents a dual-boiling, shell-and-tube, mixed working-fluid cycle. The working fluid entering the low-pressure heater is half isobutane and half propane. The condenser bubble point and dew point are 90.5 and $104^{\circ} \mathrm{F}$; the pinch point is $9^{\circ} \mathrm{F}$.

2.3.3 Discussion of Results. As previously mentioned, the results of the power balance calculations for the cycles screened in this phase of the effort are shown for the direct-contact cycles in Table I, and for the shell-and-tube cycles in Table II; numerous comparisons can be made using the results shown.

To simplify the review of the tables, a summary of some of the more significant results, in statement form, have been assembled and presented. The first seven statements pertain to the direct-contact cycles screened; the last three refer to the shell-and-tube cycles, and to their comparisons with the direct-contact cycles.

The results of screening for direct-contact cycles are: 
(1) Staged condensing resulted a $6.4 \%$ gain in net geo-fluid effectiveness (watt-hours of net plant output per pound geo-fluid) for pentane cycles.

(2) Triple boiling resulted in a gain of $8.4 \%$ in net geo-fluid effectiveness relative to dual boiling for pentane cycles.

(3) The combination of triple boiling and triple condensing for an isobutane cycle resulted in a $20 \%$ gain in net geo-fluid effectiveness relative to dual boiling and single condensing.

(4) Use of a wet cooling tower in combination with a direct-contact condenser introduces major parasitic penalties, associated with pumping cooling water to the condenser pressure, as well as complicating working-fluid recovery.

(5) For direct-contact cycles having shell-and-tube condensers, but without optional power-recovery expanders, isobutane and pentane produced comparable net geo-fluid effectiveness. A triple-boiling, triple shell-and-tube condensing isobutane cycle with power-recovery expanders showed a gain in effectiveness of $8 \%$ relative to a comparable pentane cycle.

(6) Pentane produced higher geo-fluid effectiveness than did isobutane, for cycles which combine direct-contact condensing with a wet cooling tower. However, such a pentane cycle is expected to result in a slightly lower net geo-fluid effectiveness than a comparable isobutance cycle with a shell-and-tube condenser.

(7) Mixed-hydrocarbon working fluids showed significant increases in net plant power, relative to either pure fluid in dual-boiling cycles (up to 18\%). The advantages are expected to diminish for triple-boiling and -condensing cycles. Because fluid mixtures condense over a range of temperatures at constant pressure, the cooling-water temperature rise during - condensing must be tailored to the working fluid for optimum cycle performance. The smaller temperature differences between fluids during heat addition and rejection, for mixed working fluids, shows some thermodynamic equivalence to additional stages of heat exchange. It should be noted that optimal use of mixed working fluids requires countercurrent-flow boiling condensing. This requirement could present additional problems in designing direct-contact boilers and condensers.

The results of screening for shell-and-tube cycles are:

(1) Isobutane consistently produced higher net geo-fluid effectiveness than pentane for comparable shell-and-tube cycles using Raft River geo-fluid.

(2) A mixed working fluid consisting of half isobutane and half propane, when used in a dual-boiling, single-condensing cycle, resulted in a net geo-fluid effectiveness $14 \%$ higher than that of a comparable isobutane cycle having the same heat exchanger pinch points. The net geo-fluid effectiveness of the same mixed fluid cycle was within $2 \%$ of that of a triple-boiling, triple-condensing isobutane shell-and-tube cycle having the same heat exchanger pinch points. Use of mixed-hyrodcarbon working fluids in triple-boiling, triplecondensing cycles is not expected to show any appreciable advantage over use of a pure working fluid; for the mixed-fluid, dual-boiling cycle almost $90 \%$ of the gross power was generated by the high-pressure turbine.

(3) Shell-and-tube cycles produce higher net geo-fluid effectiveness than do comparable direct-contact cycles having the same working fluid and heat exchanger pinch points (exclusive of working-fluid recovery considerations). Higher parasitics for direct-contact cycles, particularly higher geo-fluid pumping losses, account for most of this difference. The presence of a small amount of water boiled along with the working fluid in the directcontact boiler accounts for a small difference in geo-fluid effectiveness (on the order of $1 \%$ ). 


\section{WORKING-FLUID RECOVERY}

\subsection{Background}

The need for recovering working fluid in the direct-contact cycles has been introduced in Section II-2.2. That section has discussed the sources of working-fluid loss, and methods considered and/or evaluated for reducing the loss to an acceptable magnitude. In this section (Section IV-3), results of evaluation of several working-fluid recovery approaches are shown. Both mechanical systems or processes, which consist of flashing, compressing, refrigerating etc. of fluids; and chemical processes, which include dissolving, adsorbing, gettering, distilling, etc. of fluids have been considered. The evaluation effort has been more extensive for the mechanical approaches since those currently have appeared more favorable from an overall standpoint.

\subsection{Equivalence of Losing Working Fluid}

It is recognized that in a direct-contact cycle, the magnitude of working-fluid loss which is acceptable can be selected from different viewpoints, two of which are economic and environmentalistic. The question was addressed in a preliminary and somewhat arbitrary fashion for the present study.

One point of view is to relate this loss to the amount of working fluid which could fire a boiler in a steam turbine electrical power plant, and which could produce about $1 \%$ of the geothermal plant power. The first specific question answered was "How much pentane is required to fire a boiler for a $0.05-\mathrm{MW}$ steam turbine cycle?",

From Reference 7, a rather optimistic ratio of heat into the cycle to power out was taken as $7500 \mathrm{Btu} / \mathrm{kWhr}$. For a lower heating value of pentane of $19500 \mathrm{Btu} / \mathrm{lbm}$, and a boiler efficiency of $75 \%$ (based on the lower heating value), $0.05 \mathrm{MW}$ requires about $25 \mathrm{lbm} / \mathrm{hr}$ pentane. This value, $25 \mathrm{lbm} / \mathrm{hr}$, might be interpreted as an acceptable working-fluid loss rate for a 5-MW geothermal binary plant.

A second point of view might be to consider the cost of the lost working fluid. The second specific question answered was "How much pentane corresponds to $1 \%$ of the electricity costs for a 5-MW plant?"

Assuming electricity costs 50 mills/kWhr and that pentane costs 50 cents/gal and weighs $5 \mathrm{lbm} / \mathrm{gal}$, about $25 \mathrm{lbm} / \mathrm{hr}$ of pentane corresponds to $1 \%$ of electricity costs for a $5-\mathrm{MW}$ plant.

In several of the figures of the report, effects of working-fluid loss are shown parametrically to illustrate the effect of this loss on cycle state points, and on the power penalties for working-fluid recovery. For many of the calculations, to reduce the effort, specific values of fluid loss were selected and held constant for comparisons of different recovery methods. For a 5-MW gross plant, the values selected correspond to about $25 \mathrm{lbm} / \mathrm{hr}$ working fluid vented to the atmosphere from condenser-vent vapors, $6 \mathrm{lbm} / \mathrm{hr}$ reinjected with geo-fluid, and $5 \mathrm{lbm} / \mathrm{hr}$ lost from the cooling water in the wet cooling tower which was assumed for the present analyses. The magnitudes selected are somewhat arbitrary, but were chosen, in part, with the answers to the two previously posed questions in mind, and in part, because those particular loss rates resulted from calculations which had been conducted, and were close to the target values. Plant optimization, to properly allocate these losses, and to find the maximum plant net power for a given total loss, was not done here, but should be accomplished as part of future cycle optimization studies.

\subsection{Mechanical Working-Fluid Recovery Systems}

3.3.1 Introduction. The initial phase of study of Mechanical working-fluid recovery systems for the present report consisted of calculating parasitic penalties to the cycle with working-fluid loss varied parametrically. The calculations were made for a dual-boiling pentane cycle, and for recovery methods consisting of preflashing and postflashing the geothermal fluid, and venting and processing condenser vent vapors. From the initial calculations a single recovery system was selected for the dual-boiling cycle, 
and cycle power balances were calculated for an assumed total working-fluid loss for a reference dualboiling isobutane cycle, and a reference dual-boiling pentane cycle. The parametric analyses are presented in Section IV-3.3; the reference dual-boiling cycles are discussed in Sections IV-6.2 and IV-6.3.

The initial power balance calculations and some of the work done by Barber-Nichols Engineering/Lawrence Berkeley Laboratory on their $500-\mathrm{kW}$ direct-contact pilot plant pointed out improvements to the reference dual-boiling cycles. The resulting improved recovery system was incorporated in a third reference cycle, a triple-boiling, triple-condensing isobutane direct-contact cycle, in which a power balance was determined for a selected working-fluid loss. The working-fluid recovery system and parasitics for that cycle are presented in Section IV-3.3.6; the overall cycle is discussed in Section IV-6.4.

3.3.2 Approach to Analyses. The basic assumptions described for cycle screening (Section IV-2.2) were also adopted for the working-fluid recovery assessments, with an additional assumption made for treatment of dissolved liquids and vapors. For the cases analyzed, the solutions are dilute, and were assumed to follow Henry's Law. Values of the Henry constant for isobutane and pentane dissolved in geofluid were taken from Reference 2. Henry's constants for nitrogen and oxygen dissolved in water were taken from Reference 5. Henry's constant for nitrogen dissolved in working fluid was derived from page 250, Reference 6 . Use of these constants assumes equilibrium solubility. It is reported that DSS Engineers, Inc., have found in their experiments at East Mesa, that only a fraction of the amount dictated by equilibrium solubility of isobutane was dissolved in the geo-fluid (less than 50\%). For the present study, however, equilibrium solubility of working fluids and noncondensibles was assumed.

Magnitudes of the Henry constant were selected as fixed values at geo-fluid conditions representative of those for which the calculations were made. The calculations used constants having units of

$$
H^{\prime}=\frac{\text { mass fraction of gas in solution }}{\text { partial pressure of vapor (psia) }} \text {. }
$$

The values used are shown below:

\begin{tabular}{lccl} 
Solute & \multicolumn{2}{c}{$H^{\prime}$} & \multicolumn{1}{c}{$\left(\frac{\text { mass fraction in solution }}{\text { psia partial pressure }}\right)$} \\
\cline { 3 - 4 } Pentane & $1.36 \times 10^{-6}$ & Dissolved in geo-fluid \\
Pentane & $2.59 \times 10^{-6}$ & Dissolved in cooling water \\
Isobutane & $1.02 \times 10^{-6}$ & Dissolved in geo-fluid \\
Isobutane & $1.84 \times 10^{-6}$ & Dissolved in cooling water \\
Nitrogen & $1.10 \times 10^{-6}$ & Dissolved in geo-fluid \\
Nitrogen & $0.841 \times 10^{-6}$ & Dissolved in cooling water \\
Nitrogen & $32.2 \times 10^{-6}$ & Dissolved in working fluid (isobutane) \\
Oxygen & $1.74 \times 10^{-6}$ & Dissolved in cooling water
\end{tabular}

The basic calculational approach or philosophy followed for each of the parametric studies was to first make fairly detailed cycle calculations, for two or three nominal cycles. For example, multiple fluids through turbines and compressors were included in the calculation of power magnitudes, and tabulated fluid properties of water and working fluid were used. To construct the maps shown later in the report (Figures 7, 8, and 9), additional points, of course, were necessary. To obtain these, with a reasonable expenditure of effort, liberal crossplotting and interpolation of intermediate calculational quantities was done. For example, power contributions were separated and plotted versus appropriate parameters for 
which well behaved curves could be faired to cover the operating range of interest. The power contributions were then recombined to find the optimum operating conditions. To determine the effect of condenser pressure on turbine output, effective values of $\gamma$ were fitted for each turbine to the nominal back- pressure turbine calculations, and used for turbine power calculation at off-nominal condenser pressures.

3.3.3 Recovery of Working Fluid in Condenser Vent. Two parametric studies were conducted to evaluate power requirements and optimum operating conditions for recovery of working fluid from condenser-vent vapor.

(1) Compressing and Cooling-Vent Vapors. The first approach analyzed consists of: first, preheating the condenser-vent vapors to a temperature sufficiently high to prevent condensation of working fluid during compression; second, compressing the vapor mixture; third, cooling and condensing the compressed mixture using a surface condenser; fourth, venting the noncondensibles (along with a small amount of steam and working-fluid vapor) to the atmosphere; and last, discharging the condensed working fluid and water into the working-fluid feed system.

Figure 7 presents the results of the study. In the figure, the difference between the turbine power and the parasitic power loss required to compress the condenser-vent vapors is plotted against the partial pressure of nitrogen in the condenser for several values of working-fluid loss ranging from 25 to $500 \mathrm{lbm} / \mathrm{hr}$. [A nitrogen partial pressure $\left(\mathrm{P}_{\mathrm{N}_{2}}\right.$ ) of zero corresponds to a condenser pressure of 17.89 psia.] The cycle is a direct-contact, dual-boiling pentane cycle using $1.201 \times 10^{6} \mathrm{lbm} / \mathrm{hr}$ of $290^{\circ} \mathrm{F}$ geo-fluid containing 52 parts per million dissolved nitrogen. The figure illustrates an optimum condenser pressure for each of the curves of constant pentane loss. These optima have been connected by a line which passes through the point corresponding to the turbine power at zero nitrogen partial pressure (5.06 MW).

For this map the calculations assumed a compression efficiency (about 50\%) which would add enough heat to prevent pentane condensation. Separate cycle calculations were made for a point near the optimum power point at $25 \mathrm{lbm} / \mathrm{hr}$ pentane loss, which assumed a compression efficiency of $80 \%$, but with heat added to the vent vapors to prevent condensation during compression. These calculations showed an improvement in net power at that point of $0.02 \mathrm{MW}$, relative to the approach using the less efficient compressor. The heat for the process was assumed to be supplied by the geo-fluid leaving the low-pressure heater, as shown in Figure 6, with a negligible reduction in the geo-fluid temperature $\left(0.05^{\circ} \mathrm{F}\right)$.

For a cycle containing a wet cooling tower in which cooling water has been flashed to recover working fluid (See Figure 6), the condenser-vent vapors will be comprised of working-fluid vapor, steam, nitrogen, and oxygen dissolved in the geo-fluid. The specific case analyzed in detail in this effort considered 52 parts per million dissolved nitrogen in the geo-fluid; the effects of cooling-water flashing were not included in the parametric study, but were estimated separately for the pentane cycle power balance shown in Table VII.

(2) Refrigerating Compressor-Vent Vapors. The second approach analyzed consists of refrigerating the condenser-vent vapors to recondense them in order that an acceptable amount of pentane is vented along with the noncondensibles. At the refrigerated temperatures the working-fluid partial pressure is reduced with a corresponding reduction in the working fluid vented to atmosphere with the noncondensible.

The calculation approach and basic assumptions are discussed briefly in Section III-7. Freon 12 was selected as the refrigerant after looking at several Freons and making a comparison calculation using Freon 114. The heat removed and the refrigeration work were assumed to be rejected to the cooling water at $105^{\circ} \mathrm{F}$, with a cooling-water temperature of $95^{\circ} \mathrm{F}$. The cooling-water temperature rise for the conditions of interest was found to be less than about $0.5^{\circ} \mathrm{F}$. 
Figure 8 shows the results of the refrigeration calculations. The plot format and plant conditions are the same as those described for Figure 7. The results are very similar to those shown in Figure 7, with similar optimum operating points and net power. The net power output from the cycle employing the refrigeration recovery system was slightly less than that from compressing and cooling the condenser-vent products. However, the most significant result was the prediction of the occurrence of refrigerator icing at a level of working-fluid loss somewhat higher than the target value of $25 \mathrm{lbm} / \mathrm{hr}$ selected for this cycle. The dashed curve in Figure 8 shows the icing limit; pentane loss rates in $\mathrm{lbm} / \mathrm{hr}$ are numbered along the curve. Hydrates of pentane may form before icing occurs; this effect was not evaluated.

3.3.4 Preflashing Geo-Fluid. A third working-fluid recovery method analyzed parametrically, consists of preflashing the geo-fluid upstream of the high-pressure boiler in order to prevent most of the dissolved noncondensibles from entering the binary cycle. The flashing process releases a combination of steam and a fraction of the dissolved noncondensibles from the geo-fluid, the remaining geo-fluid being utilized in the binary cycle. The mass and energy balance relationships used in the flash calculations are described in Section III-6.

Figure 9 shows turbine power minus incremental geo-fluid delivery pump power for fixed total geo-fluid flow, plotted as a function of the amount of steam flashed for several magnitudes of lost pentane. As more steam is flashed, turbine power tends to be reduced because the geo-fluid flow to the cycle is reduced, and because the enthalpy of the geo-fluid utilized by the cycle is reduced. At the same time, the geo-fluid pumping power is increased because the flashing pressure decreases as more steam is flashed, and the geo-fluid must be pumped to the fixed boiler pressure. (Note that at the zero point of the abscissa, an incremental pumping power exists relative to case where no geo-fluid pressure reduction by throttling occurs.) However, as flashed steam is increased (and less noncondensibles enter the condenser) the condenser pressure can be reduced for a given working-fluid loss rate, creating a tendency for turbine power to be increased. For this figure, the condenser pressure was adjusted to obtain the specified working-fluid loss rate, at a given preflash, such that no additional condenser-vent flow processing was needed. Boiler temperatures and geo-fluid conditions are the same as those assumed for Figures 7 and 8 . Figure 9 shows clear optimum flash conditions for each pentane loss rate. These optima have been connected by a curve which passes through the turbine power minus the incremental geo-fluid delivery pump power at zero flashed steam.

In order to reduce the penalty imposed on the cycle by a geo-fluid preflash, the Lawrence Berkeley Laboratory suggested use of a heat-recovery evaporator to boil a fraction of the working fluid, using energy from the flashed vapors. This scheme, introduced in Section II-2.2 and shown in Figure 5, was evaluated as it applies to the reference 5-MW dual-boiling, direct-contact pentance cycle. Figure 10 shows the results of this evaluation; the penalty to the cycle in watt-hr/lbm geo-fluid and the fraction of the highpressure turbine flow boiled in the shell-and-tube evaporator are plotted as functions of the weight flow of nitrogen entering the boiler. An auxiliary abscissa is included to illustrate the fraction of the original amounts of dissolved nitrogen entering the boiler. The penalty to the cycle consists of the geo-fluid pumping power required to make up for the pressure drop of the flash, plus the loss in turbine power (relative to the turbine power without a preflash) associated with the change in geo-fluid enthalpy caused by the preflash. The curve shows, for example, that for a $1^{\circ} \mathrm{F}$ change in temperature during the flash, about $3 \%$ of the nitrogen originally dissolved in the geo-fluid enters the boiler (and condenser), and the penalty to the cycle is only 0.05 watt-hr/lbm geo-fluid. For this preflash, the compression power required to recover a reasonable amount of working fluid (all but $25 \mathrm{lbm} / \mathrm{hr}$ ) from the condenser-vent vapors would be negligible if the vapors were recondensed with cooling water direct from the cooling tower. When this penalty of 0.05 watt-hr/lbm is compared to the penalty shown in Figure 9 at a working-fluid loss of $25 \mathrm{lbm} / \mathrm{hr}$ (4.93 $-4.55=0.38 \mathrm{MW}$ or $0.32 \mathrm{watt}-\mathrm{hr} / \mathrm{lbm}$ geo-fluid), it is clear that a major improvement is achieved by using the heat-recovery evaporator. The improved reference direct-contact cycle, shown in Figure 5 and evaluated as discussed in Section IV.6-4, includes the heat-recovery evaporator as part of the workingfluid recovery system. 
3.3.5 Recovery of Dissolved Working Fluid. Two categories of dissolved working fluid were considered. These are working fluid dissolved in geo-fluid in the direct-contact heaters, and working fluid dissolved in cooling water when a direct-contact condenser is used in combination with a wet cooling tower.

(1) Recovery of Working Fluid Dissolved in Geo-Fluid. The method assessed parametrically, in the present study, for recovery of dissolved working fluid from geo-fluid, consists of: (a) flashing the geo-fluid after it leaves the low-pressure heater, (b) compressing the flash products, which consist of working-fluid vapor and steam, and discharging them into the condenser, and (c) pumping the flashed geo-fluid to conditions at which it is returned to the field system. For these calculations the condenser was assumed to be at $18.24 \mathrm{psia}$, and the geo-fluid return pressure was taken as $100 \mathrm{psia}$; the working fluid was assumed to be pentane.

Figure 11 shows the power penalty to the cycle plotted as a function of the weight flow of pentane reinjected with the geo-fluid. This power penalty consists of the extra power required to pump geo-fluid from the flash pressure, rather than from the heater pressure, to $100 \mathrm{psia}$, and of the power required to pump the mixture of working-fluid vapor and pentane from the flash pressure to the condenser pressure. The flash pressure is shown on the right-hand scale as a function of pentane loss rate. The basic cycle conditions are the same as those assumed for Figures 7 through 10.

Figure 11 shows that a large power penalty would exist for very low pentane loss rates, but a knee of the curve exists around 5 or $6 \mathrm{lbm} / \mathrm{hr}$ pentane loss with a parasitic power loss of about $0.06 \mathrm{MW}$ assignable to recover all but $6 \mathrm{lbm} / \mathrm{hr}$ of working fluid dissolved in the geo-fluid. Vapor compression is not required at a pentane loss above about $20 \mathrm{lbm} / \mathrm{hr}$; without any recovery the pentane loss would reach about $80 \mathrm{lbm} / \mathrm{hr}$ for the 5-MW plant.

(2) Recovery of Working Fluid from Cooling Water. Consistent with the use of direct-contact condensers with wet cooling towers, working-fluid recovery methods must be evaluated which remove dissolved working fluid from the cooling water before the cooling water enters the wet tower. Essentially all remaining dissolved working fluid will be lost to the atmosphere after the cooling water reaches the wet tower.

The approach evaluated in the present effort consists of flashing the cooling water leaving the condenser to a pressure at which only $5 \mathrm{lbm} / \mathrm{hr}$ working fluid remains dissolved in the cooling water (see Figure 6). The present calculations assumed that nitrogen and oxygen, which have dissolved in the cooling water during its residence in the tower, will come out of solution, and will accompany the working-fluid vapor and steam to the condenser. The vapor compression from the cooling water flash tank to the condenser was calculated as the compression of a mixture of ideal gases having an isentropic compression efficiency of $80 \%$. Because of the relatively small compression power involved, that treatment was judged to be reasonable. The extra power required by the condenser-vent compression pump in handling the extra flow resulting from the additional noncondensibles was estimated to be proportional to the extra noncondensible flows entering the condenser, and was based on the initial condenser vent compression calculations discussed in Section IV-3.3.

For the cooling-water flash approach, shown in Figure 6 and discussed here, almost all of the dissolved nitrogen and oxygen will come out of solution in the condenser, and will not reach the cooling-water flash tank if equilibrium concentrations are reached. Under those conditions, the vapor compression from the cooling-water flash would not include nitrogen and oxygen to any significant extent; therefore, the present calculation of the power required for vapor compression tends to be conservative. In any case, essentially all of the nitrogen and oxygen dissolved in the cooling water must pass through the condenser-vent line.

A more complicated arrangement for the recovery of working fluid dissolved in cooling water, which may be preferable to the one evaluated, includes a second cooling-water flash immediately downstream of the cooling tower. This flash would remove the dissolved nitrogen and oxygen. The noncondensible free 
cooling water would then be pumped to the condenser, and would not impose additional condenser-vent processing requirements as would the approach evaluated here (Figure 6). The oxygen, nitrogen, and water vapor from the flash would be pumped to the atmosphere and vented.

One method considered for avoiding loss of working fluid dissolved in cooling water (with a directcontact condenser and wet cooling tower) is to introduce a water loop between the direct-contact condenser and wet tower. The water loop would transfer energy to the wet cooling tower with a shell-and-tube heat exchanger and provide the direct-contact cooling medium for the condenser. An extra water pumping parasitic loss would be introduced by the loop, but the major influence would be to introduce an additional pinch point between the condenser and cooling tower. Assuming a $10^{\circ} \mathrm{F}$ pinch point for the extra heat exchanger, the loss in turbine power would be 0.5 or $0.6 \mathrm{MW}$ for a $5-\mathrm{MW}$ gross plant. This approach was not investigated further at this time since the parasitic losses for flashing cooling water to recover working fluid were estimated to be of the order of magnitude or smaller than losses introduced by the additional water loop.

3.3.6 Working-Fluid Recovery Parasitics for a Triple-Boiling, Direct-Contact Isobutane Cycle. The working-fluid recovery analyses discussed in the preceding paragraphs (IV-3.3.3 through IV-3.3.5) have led to the selection of a mechanical recovery process for the improved, reference, directcontact isobutane cycle. This cycle is discussed in Section IV-6.4, and its schematic is shown in Figure 5. Table III includes the parasitic losses estimated for the several components of this recovery system in units of watt-hr/lbm geo-fluid and in terms of percent of net plant power. (The table also includes the magnitudes of power recovered by three expanders which can be introduced as part of the working-fluid recovery system. These magnitudes are discussed in Section IV-4.)

The first step in the selected working-fluid recovery process is to preflash the geothermal fluid through a $1^{\circ} \mathrm{F}$ temperature drop; correspondingly, the pressure drops from 100 to $58 \mathrm{psia}$. The resulting vapor, which contains $97 \%$ of the initial 52 parts per million nitrogen dissolved in the geothermal fluid, is passed through a shell-and-tube heat exchanger in which $5.7 \%$ of the working fluid passing through the highpressure heater is evaporated. Condensed water at $278^{\circ} \mathrm{F}$ is returned to the cycle upstream of the geo-fluid delivery pump. The remaining vapor is shown as being vented to the atmosphere through an optional vapor expander. Table III shows that the parasitic loss for this phase of the recovery amounts to about $1.1 \%$ of net plant power.

With all but $3 \%$ of the dissolved nitrogen removed in the preflash, the condenser-vent problem is minimized; the nitrogen partial pressure in the condenser was assumed to be 1.0 psia. As shown in the schematic, the remaining nitrogen is removed from the system by venting the high-pressure turbine condenser through a heater and a vapor compressor to a shell-and-tube recovery condenser. The cooling water passes through the recovery condenser before passing through the main condenser, resulting in negligible power required for the vapor compressor for a working-fluid loss at this point of $25 \times 10^{-6}$ times the geothermal fluid flow. The condensate from the recovery condenser is piped to the working-fluid feed line. (This approach was chosen over one in which nitrogen leaves the condenser dissolved in the working-fluid condensate, and then leaves the system by becoming redissolved in the geo-fluid in the low-pressure, directcontact preheater. The reason for the choice is based on the low nitrogen pressure in the condenser, and the fact that nitrogen is about one-thirtieth as soluble in water as in working fluid.)

Recovery of working fluid from the spent geothermal fluid is accomplished by flashing it before reinjection. The vapors from the flash, which contain steam and working fluid, are compressed and fed into the low-pressure turbine condenser. As is shown in Table III only about $0.1 \%$ of the net plant power is required for this compression.

The spent geo-fluid is pumped to the plant outlet pressure, which was assumed to be 100 psia; the power required was estimated to be 0.10 watt-hr $/ \mathrm{lbm}$ geo-fluid. Had the geo-fluid not been flashed before reinjection, its pressure would have been $145.7 \mathrm{psia}$, and $0.06 \mathrm{watt}-\mathrm{hr} / \mathrm{lbm}$ credit would have been given to the cycle to account for a reduced injection pumping requirement. Therefore, the effective cycle penalty 
assigned to postflash geo-fluid pumping is $0.10+0.06=0.16$ watt-hr $/ \mathrm{lbm}$ geo-fluid or $3.6 \%$ of the net cycle power. The working fluid reinjected with the geo-fluid is $5 \times 10^{-6}$ times the geo-fluid.

The total loss of working fluid estimated for the cycle is $30 \times 10^{-6}$ times the geo-fluid flow. The total penalty to the cycle for this working-fluid recovery process is $1.1+0.1+3.6=4.8 \%$ of the net plant power. However, as Table III shows and as will be discussed in Section IV-4, almost half of this penalty could be recovered using expanders, with a resulting net penalty estimated at $2.7 \%$ of the net power. Further, for the same total working-fluid loss, the distribution of losses could be improved by reducing the strength of the preflash and postflash, reducing the nitrogen partial pressure in the condenser, and increasing the condenser-vent compression power. With these improvements, it is estimated that the net penalty for recovering working fluid could be reduced to perhaps $2 \%$ of net plant power.

\subsection{Chemical Working-Fluid Recovery Systems}

3.4.1 Introduction. A preliminary evaluation of several chemical working-fluid recovery approaches was made by Energy Inc., Idaho Falls, Idaho (EI) under subcontract to EG\&G Idaho, Inc. The approaches considered under this contract applied mainly to recovery of working fluid from condenservent vapors while venting the nitrogen to the atmosphere. However, these same approaches, when combined with stripping or sparging processes, can be applied to the recovery of working fluid dissolved in the geo-fluid, as discussed in Section II-2.2. E. Hoffman, Energy Consultant, Laramie, Wyoming worked with EG\&G Idaho, Inc., in the same area, briefly reviewing the EI study and introducing some additional considerations.

3.4.2 Recovery from Condenser-Vent Vapors. EI considered four basic approaches related to the working-fluid recovery problem arising from the presence of dissolved nitrogen in the geo-fluid. The first of these consists of bubbling condenser-vent vapors through a solvent which will dissolve the working fluid. The nitrogen is then vented, and the working fluid is subsequently separated from the solvent by distillation. It was estimated that equipment of substantial size would be required for the process, and that a solvent flow rate would be of the same order of magnitude as the working-fluid flow rate for the cycle. In addition, and perhaps more importantly, parasitic losses were estimated to total perhaps $11 \%$ of net plant power, the major contributor being the energy extracted from $290^{\circ} \mathrm{F}$ geo-fluid to separate the working fluid from the solvent by distillation.

The second process considered consists of passing the condenser-vent vapors over a charcoal bed which would adsorb the working-fluid vapor, allowing the nitrogen to be vented. The bed would subsequently be regenerated, and working, fluid would be recovered by application of heat taken from $290^{\circ} \mathrm{F}$ geo-fluid. Again, substantial equipment sizes were estimated, and perhaps $8 \%$ of net plant power was estimated as a parasitic penalty.

The third and fourth approaches consisted of removing nitrogen from the condenser-vent vapors, and from the geo-fluid, directly, by chemical gettering. These approaches were concluded not to be feasible at present.

3.4.3 Recovery of Dissolved Working Fluid. Chemical systems considered for recovery of dissolved working fluid from the spent geo-fluid or from cooling water must compete with a mechanical system which consists of flashing the geo-fluid or cooling water. Passing the geo-fluid directly through a working-fluid solvent would require equipment which can handle the large geothermal or cooling-water flows, and in addition, would entail replacing solvent which became dissolved in the water and either lost to the atmosphere in a wet cooling tower or reinjected with the geo-fluid. This approach was judged not to be superior to (or even competitive with) the mechanical approach of flashing the spent geo-fluid. Passing the entire geo-fluid or cooling-water flow through an adsorbent bed was also judged to be impracticable.

A sparging process, using perhaps air, could be applied to the geo-fluid and/or cooling water, initially, followed by one of the processes discussed in the preceding section, to separate and recover the working 
fluid from the air which would be mixed with the working fluid. Those latter processes would suffer, in principle, from the same disadvantages discussed relative to recovery from the condenser-vent vapors (in Section IV-3.4.2), although the magnitudes of working-fluid flows to be recovered for this application could be much smaller. If air were the sparge gas, flammable mixtures could be created; sparge-gas makeup would be a problem if inert gases were used. Beyond these qualitative arguments, chemical schemes for recovering dissolved working fluid were not evaluated.

3.4.4 Conclusions Relative to Chemical Systems for Working-Fluid Recovery. The review of the Chemical systems made by E. Hoffman generally agreed with the study made by EI. However, extending Mr. Hoffman's review of the system involving bubbling the condenser-vent vapors through a solvent, to an assessment of parasitic penalties of that system, resulted in smaller penalties to the cycle than did EI's analysis. The penalties still appear to exceed those imposed by the mechanical system evaluated.

Mr. Hoffman's review included preliminary consideration of the use of a molecular sieve bed for adsorbing nitrogen, thus separating it from the working-fluid vapor in the condenser vent. The potential advantage of the molecular sieve approach, relative to use of the working-fluid solvent or the bed which adsorbs working fluid, lies in its treatment of only the nitrogen flow, which is a small fraction of the condenser-vent vapor flow. A molecular sieve for this application is apparently not an off-the-shelf item, and its use would be complicated by the presence of some carbon dioxide dissolved in the geo-fluid as well as nitrogen. Further, concern was expressed relative to sieve damage, regarding the use of steam (the most readily available energy source) for its regeneration.

The conclusion was reached that none of the chemical working-fluid recovery approaches had been demonstrated to be competitive with the mechanical system selected and discussed in Section IV-3.3.6.

\section{POWER-RECOVERY EXPANDERS}

\subsection{Background}

As discussed in Section II-2.3, power-recovery expanders can be used to recover power from the geofluid as it undergoes pressure drops during several processes in a direct-contact cycle. In the basic cycle, geo-fluid pressure drops occur between the higher and lower boiler/heater pressure levels; in the workingfluid recovery system, pressure drops are associated with the flashing of geo-fluid or cooling water, and expansion of the flashed geo-fluid vapor.

Different values of isentropic efficiency were assumed for the several expanders evaluated. An efficiency of 0.7 was assumed for an expander having a saturated water-vapor inlet flow (preflash vapor expander). For saturated liquid inlet conditions the efficiency was assumed to equal 0.6 (preflash and postflash geofluid expander or cooling-water flash expander). An expander in which driving fluid was maintained subcooled throughout, was assumed to have an efficiency of 0.8 (expanders between different boiler/heater pressure levels).

\subsection{Power Recovered from Basic Cycle}

Magnitudes of power recovered through use of expanders in the basic cycles have been calculated and included in the individual power balance summaries for the dual-boiling isobutane and pentane cycles, and the triple-boiling isobutane direct-contact cycle shown in Tables VII, IX, and XI, and discussed in Section IV-6. A summary of the effectiveness of these expanders is contained in Table IV. Table IV presents recoverable power (in units of watt-hr/lbm geo-fluid) from expanders placed in the geo-fluid lines between the high-pressure heater and low-pressure boiler for dual-boiling cycles, and between high- and medium-pressure boilers/heaters, and medium and low-pressure boilers/heaters for the triple-boiling isobutane cycle. Magnitudes of power recovered range from about $1.4 \%$ of net plant power for the lowest pressure cycle (pentane) to over $6 \%$ for the triple-boiling isobutane cycle. 


\subsection{Expanders in the Working-Fluid Recovery System}

The power derivable using expanders in the working-fluid recovery system has been estimated for the three, reference, direct-contact cycles mentioned in the preceding paragraph. These values are included in Tables VII, IX, and XI. Table III contains the contributions to recovered power from the working-fluid recovery system of the triple-boiling isobutane cycle. Table III shows that almost half of the working-fluid recovery parasitic penalty for this cycle can be recovered by the power-recovery expanders; the most significant recovery results from flashing the spent geo-fluid through a hydraulic expander rather than a throttling orifice.

\section{PRELIMINARY ASSESSMENT OF HEAT-REJECTION SYSTEMS}

\subsection{Dry Cooling Tower Considerations}

The present effort has not emphasized the study of heat-rejection systems, however, some preliminary thoughts have been given to their effects on cycle behavior. A cycle using a direct-contact condenser, suffers an extra burden when a wet tower is used; that burden consists of the loss of working fluid dissolved in cooling water to the atmosphere from the wet tower. The working fluid dissolves in the cooling water while in the direct-contact condenser. A preliminary estimate of parasitic power required for a dry tower was made consistent with the approach suggested in Reference 8. The estimate indicated that more than $2 \mathrm{MW}$ are required for a 5-MW gross plant to supply fan and cooling-water pumping power for a dry tower which could provide the same condensing temperature as assumed for the wet tower. The power required for the wet tower was estimated to be about $0.5 \mathrm{MW}$ (Reference 1); therefore, the power penalty for that dry tower is $1.5 \mathrm{MW}$.

Because of the large magnitude of the parasitic power required for a dry tower, a plant using a dry tower and optimized for maximum geo-fluid utilization, will have a higher condensing temperature than a plant using a wet tower. A smaller dry tower, requiring perhaps $1 \mathrm{MW}$, might correspond to an increase in condensing temperature of about $10^{\circ} \mathrm{F}$, which was shown to reduce the turbine power in the 5 - $\mathrm{MW}$ (gross) plant by about $0.5 \mathrm{MW}$. Therefore, the penalty assignable to the smaller dry tower would be reduced to about $1.0 \mathrm{MW}$, rather than the $1.5 \mathrm{MW}$ estimated for the wet tower condensing temperature. With additional increases in condensing temperature, further reductions in power for a dry cooling tower could be attained, but the turbine power would be reduced further. Optimization has not been done in this study, but the power penalty for a conventional dry tower is expected to be in excess of $0.5 \mathrm{MW}$ for a 5-MW gross cycle. For the present effort, therefore, it was concluded that all remaining cycle comparisons would be made assuming wet towers; power penalties required to prevent serious working-fluid loss did not exceed 0.5 -MW by a significant amount. The ecological advantages of a dry tower are recognized, but study of those trade-offs is considered to be a separate subject, and is not treated here.

\subsection{Comparison of Several Heat-Rejection Systems}

5.2.1 Background. As previously mentioned, the cycle calculations made in the present study assumed the use of wet cooling towers, combined with either direct-contact or shell-and-tube condensers. However, a preliminary comparison of both spray-augmented dry cooling towers (for use with directcontact condensers) and evaporative condensers was made relative to those systems for which the cycles were analyzed. The basic information required to conduct the comparison came from: (a) the wet cooling tower specifications provided by the Marley Cooling Tower Company for the first Raft River 5-MW Geothermal Pilot Plant (b) Baltimore Aircoil of California, and (c) The Niagara Blower Company.

The terminology "spray-augmented dry tower" as used, in this report refers to a cooling tower in which the plant cooling water is cooled inside tubes using a combination of atmospheric air forced past the outside of the tubes, and water sprayed on the outside of the tubes to augment the cooling; spray water is used as required by ambient temperature. In an evaporative condenser the plant cooling water in the sprayaugmented dry tower is replaced by the turbine exhaust vapors which are condensed directly in the tubes. 
Both systems isolate the atmosphere and the water spray used to augment cooling from the remainder of the binary cycle, as would a dry cooling tower.

5.2.2 Heat-Rejection Systems Considered. The parasitic penalties to several dual-boiling, directcontact cycles are shown in Table V. As a reference case, an isobutane cycle was chosen which included a wet cooling tower and a shell-and-tube condenser having a $9^{\circ} \mathrm{F}$ pinch point. The fan power plus water pumping power for the cooling tower was estimated to correspond to $0.45 \mathrm{watt}-\mathrm{hr} / \mathrm{lbm}$ geo-fluid. (This loss is essentially equivalent to the corresponding values from Reference 1 , which were used in the cycle analyses of Sections IV-2 and IV-6.)

The next case compared consists of a pentane cycle with a wet cooling tower and a direct-contact condenser having a $5^{\circ} \mathrm{F}$ pinch point. The basic cooling-tower parasitic loss, including the effect of pumping the cooling water from the tower to the condenser, was estimated to be 0.44 watt-hr/lbm geo-fluid. The reduced pinch point assumed for the direct-contact condenser was estimated to result in a reduced condenser pressure, and a turbine power which was increased by 0.21 watt-hr/lbm geo-fluid, relative to the reference case. At the same time, an increment in parasitics of 0.26 watt-hr/lbm was incurred because of the additional working-fluid recovery problem imposed by the direct-contact condenser. The equivalent parasitic loss for this heat rejection system was estimated to be $0.44-0.21+0.26=0.49 \mathrm{watt}-\mathrm{hr} / \mathrm{lbm}$.

Similarly, parasitic losses are presented for four isobutane cycles having: (a) a wet tower and a directcontact condenser, (b) a spray-augmented dry tower and a direct-contact condenser, (c) an evaporative condenser having a $104^{\circ} \mathrm{F}$ condensing temperature (equal to the condensing temperature of the reference case), and (d) an evaporative condenser having an $84^{\circ} \mathrm{F}$ condensing temperature. All of the cases compared assumed a $65^{\circ} \mathrm{F}$ ambient wet-bulb temperature.

5.2.3 Results of the Comparison. Table V shows that the best two of the heat rejection systems reviewed are the evaporative condenser and the combination of a wet tower with a shell-and-tube condenser for the isobutane cycle. Closely approaching them is the wet tower combined with a direct-contact condenser for a pentane cycle. Poorer, by a significant increment in equivalent parasitic losses, were the spray- augmented dry tower, and the combination of a wet tower with a direct-contact condenser for isobutane.

It should be noted that if a triple condenser had been used to replace the single condenser in the reference heat-rejection case, the turbine power would have been about $0.35 \mathrm{watt}-\mathrm{hr} / \mathrm{lbm}$ larger. Therefore, for a triple-boiling isobutane cycle, for example, the evaporative condenser and the shell-and-tube condenser combined with a wet cooling tower appear to be quite competitive from the standpoint of net geo-fluid effectiveness. Economic analysis must be included in a final selection of a heat-rejection system.

\section{REFERENCE CYCLES}

\subsection{General}

Of the cycles analyzed during the present study, four were selected as reference cycles, and are presented in this section. The first two, consisting of dual-boiling, single-condensing, direct-contact isobutane and pentane cycles, were reviewed with the intent of understanding and evaluating direct-contact cycles which are comparable in basic structure to the dual-boiling, shell-and-tube isobutane cycle of the first Raft River 5-MW Pilot Plant. Preliminary working-fluid recovery systems were assessed for these cycles; a schematic is shown in Figure 6. The third reference cycle is a triple-boiling, triple-condensing, direct-contact isobutane cycle; the schematic is shown in Figure 5. An improved working-fluid recovery system was assessed for this cycle. The fourth reference cycle is a triple-boiling, triple-condensing, shell-and-tube isobutane cycle. This cycle was selected to compare with the corresponding direct-contact cycle. Major state points for the cycles, as well as cycle power balances, are shown in sections following. 


\subsection{Dual-Boiling, Direct-Contact Pentane Cycle}

Analysis of this basic cycle consisted of the calculation of state points and power balances for several cycles which incorporated different working-fluid recovery methods. The working-fluid recovery methods were evaluated for a range of working-fluid losses. The results are presented in this section as a nominal set of cycle state points and power balance (which could not actually be completed until after the workingfluid recovery methods were studied), and in Section IV-3.3 as maps describing the performance trade-offs associated with the recovery methods investigated.

6.2.1 Basic Cycle State Points. Table VI shows the cycle state points for a dual-boiling, directcontact pentane cycle using Raft River geo-fluid $\left(290^{\circ} \mathrm{F}, 52\right.$ parts per million nitrogen). The flows shown are for a 5-MW gross plant, with a working fluid loss rate of $36 \mathrm{lbm} / \mathrm{hr}$. The plant is assumed to include a single direct-contact condenser and the working-fluid recovery equipment shown schematically in Figure 6. (Exceptions are the preflash tank and expander which were not included for the cycle represented in Table VI.)

In Figure 6 (and for calculational purposes) the boilers and heaters were shown separately. It is assumed that for a real plant a boiler/heater combination may well be a single integrated component. The state point numbers refer to the numbers shown in Figure 6; these numbers are not necessarily consecutive. It should be noted that calculation of actual temperatures of vapor mixtures following expansion or compression was not required; enthalpies were calculated. The footnoted temperatures in Table VI are the isentropic compression or expansion temperatures; the actual temperatures are somewhat higher than those shown. Because the calculations were made by hand, iteration between the working-fluid recovery portion of the cycle and the boiler/heater/turbine portion was not completed. Accordingly, some small inconsistencies can be expected in mass and/or energy balances.

6.2.2 Power Balance. The power balance and power penalties, relative to a shell-and-tube cycle, are shown in Table VII for the pentane direct-contact, dual-boiling cycle using $1.201 \times 10^{6} \mathrm{lbm} / \mathrm{hr}$ of Raft River geo-fluid $\left(290^{\circ} \mathrm{F}\right.$ and 52 parts per million dissolved nitrogen). The cycle was sized at 5-MW turbine power with a condenser pressure of 18.24 psia, which corresponds to a condensing temperature of $104^{\circ} \mathrm{F}$ with a partial pressure of nitrogen in the condenser of 0.35 psia. The turbine and parasitic power values shown, are basically for the cycle whose state points are shown in Table VI. Nominal boiler/heater and condenser pinch points were selected as 10 and $9^{\circ} \mathrm{F}$, respectively, for this cycle. Working-fluid losses were held to $36 \mathrm{lbm} / \mathrm{hr}$. Boiler temperatures of 230 and $170^{\circ} \mathrm{F}$, for the high- and low-pressure boilers, were selected from Reference 1. Calculations assessing the working-fluid recovery power requirements (discussed in Section IV-3.3), indicated that operation of a partial pressure of nitrogen in the condenser near 0.6 psia rather than the 0.35 psia for which state points were calculated is closer to an optimum power balance; the power values shown in Table VII correspond to this higher condenser pressure. As mentioned in Section IV-2.2, it has been assumed that geo-fluid is delivered to the plant at 100 psia and returned to the field at 100 psia.

The first part of Table VII shows the magnitudes of power from the turbine and three expanders. Use of the expanders is optional; a trade-off must be made between cycle complexity/cost and performance. The incremental power attainable, at the same geo-fluid flow, by reducing pinch points in the direct-contact heat exchangers is shown. The pinch points in the boilers/heaters were changed from 10 to $5^{\circ} \mathrm{F}$; in the condenser from 9 to $5^{\circ} \mathrm{F}$.

In the second part of Table VII, the various parasitic losses are shown. The basic wet cooling-tower loss was estimated, based on cooling-water flow and temperature increments, from Reference 1. That loss represents the power to operate the cooling tower without working-fluid recovery requirements. The last three items in the table represent increments in power associated with flashing cooling water to reduce loss of working fluid in the wet tower to $5 \mathrm{lbm} / \mathrm{hr}$. The power requirement shown for the geo-fluid reinjection pump includes the power to recover pressure after flashing the geo-fluid downstream of the low-pressure heater to remove dissolved working fluid. The geo-fluid postflash vapor pump and condenser-vent pump 
are part of the working-fluid recovery system. The turbine loss at optimum condenser pressure represents a loss associated with the increased back pressure corresponding to the partial pressure of nitrogen in the condenser at the optimum condenser pressure.

Values shown in the right-hand column of Table VII, labeled "penalties assignable to a direct-contact cycle," were obtained by first estimating the parasitic loss due to each item for a corresponding shell-andtube cycle, and showing the amount by which the direct-contact plant parasitic power exceeds the value estimated for the shell-and-tube cycle. Penalties in geo-fluid and feed pump power occur, because for the direct contact cycle both the geo-fluid and working fluid must be pumped to a pressure equal to the sum of the working fluid and geo-fluid vapor pressures at the boiler temperatures (plus a small increment for noncondensible gas partial pressure in the boilers). A significant penalty appears for geo-fluid reinjection pumping because the geo-fluid is not flashed in the shell-and-tube cycle, and was assumed to have a pressure in the low-temperature boiler which exceeded the 100 psia selected as the return pressure.

From Table VII, different values of plant net power can be estimated depending on options selected. Different pinch points can be assumed; different expander options can be assumed; utilization of a different working-fluid recovery scheme which eliminates condenser-vent pumping can be included; use of either a surface or direct-contact condenser can be assumed; or combinations of the above can be selected. For example, if a surface rather than direct-contact condenser is assumed, the last three parasitic losses can be eliminated. Noncondensibles must still be vented from the condenser, so the condenser-vent pump and turbine loss due to the presence of nitrogen in the condenser result in power penalties.

\subsection{Dual-Boiling, Direct-Contact Isobutane Cycle}

For comparison with results of the pentane-cycle analyses, presented in the preceding section, calculations of state points and power balance for a direct-contact, dual-boiling, single-condensing, isobutane cycle using Raft River geo-fluid $\left(290^{\circ} \mathrm{F}, 52\right.$ parts per million nitrogen) were conducted. Direct-contact condensing as well as boiling were assumed for the cycle. The same approach and basic assumptions as for the pentane cycle were employed. However, for this isobutane cycle, working-fluid recovery methods were examined to a lesser extent; a single recovery method and a single magnitude of loss were selected for each contributor to the loss of isobutane. In addition, two separate cycles were analyzed to answer questions arising during the principal calculations.

6.3.1 Basic Cycle State Points. Table VIII shows the cycle state points for a 5-MW (gross) isobutane plant, with an isobutane loss selected as $36 \mathrm{lbm} / \mathrm{hr}$. Figure 6 constitutes the schematic for the cycle, except that preflashing the geo-fluid was not done for these calculations. The remainder of the working-fluid recovery methods shown in Figure 6 were assumed for the isobutane cycle. As was discussed for Table VI, footnotes on several of the temperature values indicate that these are isentropic compression or expansion temperatures, rather than the actual temperatures.

The major differences between the isobutane and pentane cycle state points consist of:

(1) Higher pressures for the isobutane cycle (407 versus 128 psia) resulting in higher pumping parasitic losses and perhaps higher plant vessel costs

(2) Lower turbine exit densities for the isobutane cycle (about a factor of 4) resulting in lower turbine costs

(3) Lower geo-fluid flow for the same turbine power for the isobutane cycle (about $15 \%$ lower).

6.3.2 Power Balance. The power balance for the direct-contact isobutane cycle, and its power penalties, relative to a shell-and-tube cycle, are shown in Table IX. The cycle used $1.055 \times 10^{6} \mathrm{lbm} / \mathrm{hr}$ of Raft River geo-fluid. The cycle was sized at 5.04-MW turbine power, at a condensing temperature of 
$104^{\circ} \mathrm{F}$, and condenser pressure of 79.78 psia. The turbine and parasitic power values shown correspond to the cycle whose state points are shown in Table VIII. Total working-fluid losses were held to $36 \mathrm{lbm} / \mathrm{hr}$. Boiler temperatures were selected as 240 and $180^{\circ} \mathrm{F}$ from Reference 1 . Nominal boiler/heater and condenser pinch points were 10 and $9^{\circ} \mathrm{F}$, respectively, for this cycle.

- Table IX is organized the same as is Table VII; the discussion of the organization and content of those tables is presented in Section IV-6.2.2, and pertains equally to this section.

A comparison of Table IX with Table VII shows that for the same turbine power, the parasitic power losses for the isobutane cycle are generally higher, and the geo-fluid flow lower than for the corresponding pentane cycle.

6.3.3 Additional Analyses. Two additional calculations were made for Raft River geo-fluid conditions with the dual-boiling, direct-contact isobutane cycle to answer questions of a sensitivity analysis nature which arose during the principal calculations.

(1) Effect of lowering Low-Pressure Boiler Temperature. The primary cycle calculations (Table VIII) resulted in a geo-fluid temperature of $152^{\circ} \mathrm{F}$ leaving the low-pressure heater. As mentioned earlier, the boiler temperatures were selected for given working-fluid and geo-fluid temperatures, but for a shell-and-tube cycle. It was of interest to determine if any reason exists to believe that those optima would not be reasonably close to optima for a dual-boiling, direct-contact cycle.

To answer this question and to try to get more energy out of the geo-fluid, the low-pressure boiler temperature was lowered from 180 to $170^{\circ} \mathrm{F}$, and the cycle calculations were repeated as necessary. It was found that the temperature of the geo-fluid leaving the plant was reduced from 152 to $145^{\circ} \mathrm{F}$. The plant net geo-fluid effectiveness for the reduced boiler temperature was calculated to be within about $0.5 \%$ of that for the original temperature. This calculation did not suggest that the optimum boiler temperatures for shell-and- tube cycles are significantly different from those of direct-contact cycles.

(2) Effect of Increasing Geo-Fluid Inlet Enthalpy. The initial calculations for the isobutane cycle used a geo-fluid enthalpy of $260.09 \mathrm{Btu} / \mathrm{lbm}$ entering the high-pressure boiler. This value was determined directly from the $290^{\circ} \mathrm{F}$ geo-fluid temperature and 406.5 psia boiler pressure. When calculating parasitic losses, it was determined that a better value of inlet geo-fluid enthalpy is $260.74 \mathrm{Btu} / \mathrm{lbm}$, which would be obtained at the outlet of a pump $(\eta=0.8)$ that accepts geo-fluid at $290^{\circ} \mathrm{F}$ and $100 \mathrm{psia}$, and pumps it to 406.5 psia.

The cycle calculations were repeated through the boilers/heaters and turbines. An increment in turbine power of $0.04 \mathrm{MW}$ was estimated to correspond to the inlet enthalpy increment of $260.74-260.09=$ $0.65 \mathrm{Btu} / \mathrm{lbm}$. This correction was made to the power balance values shown in Table IX.

\subsection{Triple-Boiling, Triple-Condensing, Direct-Contact Isobutane Cycle}

6.4.1 Basic Cycle State Points. This cycle was chosen as representing a low-cost energy, directcontact cycle which could be selected today without major risk. A schematic of the cycle is shown in Figure 5. The basic cycle has three direct-contact boilers and preheaters; the boiler temperatures are 260 , 210 , and $160^{\circ} \mathrm{F}$. As discussed in Section IV-5.1, a wet cooling tower was assumed.

Use of direct-contact condensers in combination with wet towers introduces significant cooling-water pumping requirements when the condensers operate at high pressures relative to atmospheric pressures. Additionally, the working-fluid recovery problem is aggravated by potential loss of working fluid in the wet tower, and nitrogen and oxygen entering into the condenser from the wet tower. Accordingly, this reference cycle employs shell-and-tube condensers in combination with the wet cooling tower. Three shelland-tube condensers, connected to the cooling water in series, have condensing temperatures of 91,99 , and $104^{\circ} \mathrm{F}$ for the high-, medium-, and low-pressure turbine exhausts. Condenser pinch points are all $9^{\circ} \mathrm{F}$, and a nitrogen partial pressure of 1.0 psia was assumed in each condenser. 
The working-fluid recovery system (discussed in Section IV-3.3.6) consists of a geo-fluid preflash and postflash, with a working-fluid evaporator to recover energy from the preflashed geo-fluid vapor, as well as a condenser-vent compressor and cooler. This recovery system is improved relative to the systems analyzed for the previous two reference cycles (Sections IV -6.2 and IV-6.3).

Cycle state points are shown in Table $\mathrm{X}$ for boiler pinch points of $5^{\circ} \mathrm{F}$. For this cycle, all flow rates are shown in a dimensionless form, as the state-point flow divided by the geo-fluid inlet flow. Working-fluid losses for the cycle have been selected as $25 \times 10^{-6}$ and $5 \times 10^{-6}$ times the inlet geo-fluid flow for the working fluid vented to the atmosphere and reinjected, respectively. Optional power-recovery expanders, as discussed in Section IV-4, were included in the cycle, and the effects of the small amount of energy removed by these expanders have been estimated for the state point properties presented.

Since the basic cooling-tower parasitic losses were estimated as a factor times the cooling-water flow through the wet tower, detailed pressure values of the cooling water were not calculated, nor are they shown in Table X.

To avoid iterative cycle calculations associated with the triple condensers, the three condensing temperatures were selected which, hopefully, would result in the same cooling-water flow for each condenser. As is shown in Table X, the required cooling-water flows were found not to be exactly the same for the three condensers. Rather than repeat the cycle calculations, two cooling-water bypass lines were introduced to permit flows consistent with the related temperatures. These bypass lines are shown by two dotted lines in Figure 5 (one horizontal and one vertical, between the medium- and low-pressure turbine condensers). The corresponding values for total cooling-water flow and temperature rise were calculated, as shown in Table $\mathrm{X}$, and used to estimate a correction to the basic cooling-tower parasitic loss. The corrected parasitic loss is shown in the next section.

6.4.2 Power Balance. The power balance for the triple-boiling, triple-condensing, direct-contact isobutane cycle, whose state points are shown in Table X, is presented in Table XI. As previously noted, this cycle was assumed to have $5^{\circ} \mathrm{F}$ pinch points in the direct-contact boilers/heaters, and $9^{\circ} \mathrm{F}$ pinch points in the three shell-and-tube condensers.

Values of turbine power are shown in order for the high-, medium-, and low-pressure turbines, in watt$\mathrm{hr} / \mathrm{lbm}$ geo-fluid. Values of parasitic losses for the basic cycle and for working-fluid recovery are also shown. For this cycle the condenser-vent compressor requires essentially no power.

For completeness, five auxiliary expanders were introduced. The enthalpy extracted from the system by these expanders was accounted for in the cycle analysis, and amounted to about $10 \%$ of the power delivered by the expanders. The expanders were driven by: (a) the vapors generated by the preflashed geofluid $(\eta=0.7)$, (b) the actual preflash being accomplished in a hydraulic turbine rather than an isenthalpic throttling process $(\eta=0.6)$, (c) the geo-fluid dropping in pressure from the high- to the medium pressure boiler $(\eta=0.8)$, (d) the geo-fluid dropping in pressure from the medium- to the low-pressure boiler $(\eta=0.8)$, and (e) the geo-fluid postflash being accomplished in a hydraulic expander $(\eta=0.6)$. In an actual plant, probably only the last three expanders would be used. Values of auxiliary expander power are shown in Table $\mathrm{X}$ in the order discussed above; the units are watt-hr/lbm geo-fluid. Cooling-tower parasitic losses were again taken as 0.077 times the cooling-water flow, with a small adjustment included to account for an imperfect balance of cooling-water flow required by the three condensers. Additional parasitic losses associated with cooling-water makeup and working-fluid makeup were assumed negligible.

A comparison of the total turbine power for triple-boiling cycle with that for the dual-boiling cycle (Table IX), shows about a $28 \%$ improvement at the same geo-fluid flow for the triple-boiling, triplecondensing cycle. Of course, less improvement in net power is achieved.

6.4.3 Effect of Boiler Pinch Points on Cycle. The effect of changing boiler/heater pinch points on the performance was investigated for this cycle. Values of 10 and $1^{\circ} \mathrm{F}$ were investigated in addition to the 
nominal pinch points of $5^{\circ} \mathrm{F}$ selected for this cycle. Pinch points for the shell-and-tube condensers were held constant at $9^{\circ} \mathrm{F}$ for the cycle. The following summarizes the values of net plant power in watt-hr/lbm geo-fluid as a function of boiler pinch point for the triple-boiling, triple shell-and-tube condensing, directcontact isobutane cycle.

$\begin{array}{cc}\text { Boiler Pinch Points } & \begin{array}{c}\text { Net Plant Power } \\ \text { (watt-hr/lbm geo-fluid) }\end{array} \\ 10^{\circ} \mathrm{F} & 4.421 \\ 5^{\circ} \mathrm{F} & 4.781 \\ 1^{\circ} \mathrm{F} & 4.894\end{array}$

It might be noted that this cycle, with boiler pinch points of $1^{\circ} \mathrm{F}$, shows about a $19 \%$ improvement in net plant power relative to the first Raft River 5-MW Pilot Plant (Case 13, Table II).

\subsection{Triple-Boiling, Triple-Condensing, Shell-and-Tube Isobutane Cycle}

This shell-and-tube plant represents an improved binary cycle (relative to the first Raft River 5-MW Pilot Plant) which is quite competitive with the comparable direct-contact cycle in terms of net plant power. The shell-and-tube plant, however, does not require the complexity of auxiliary power-recovery expanders or working-fluid recovery components. State points, turbine power, and parasitic losses for the cycle are presented for comparison. Final cycle selection must include economic considerations, which have not yet been completed.

6.5.1 Cycle State Points. The triple-boiling, triple-condensing, shell-and-tube isobutane cycle is arranged much the same as is the basic part of the comparable direct-contact cycle shown in Figure 5. The geo-fluid passes in series through the high-pressure boiler and heater, the medium-pressure boiler and heater, and the low-pressure boiler and heater. The working fluid leaving the condenser system passes through the low-pressure heater, after which it splits, passing in part through the low-pressure boiler and in part through the medium-pressure heater. The working-fluid flow is split similarly between the mediumpressure heater and boiler. None of the working-fluid recovery components are required in this cycle. The triple-condensing system is as shown in Figure 5, except that this cycle does not require condenser venting. Pinch points were assumed to be 10 and $9^{\circ} \mathrm{F}$ for the boilers/heaters and condensers, respectively.

Because of the similarity of this cycle to the one shown in Figure 5, a separate figure was not included. State points for the shell-and-tube cycle are presented in Table XII, which is organized by components, starting with the high-pressure boiler, heater, turbine, and condenser, and progressing through the medium- and low-pressure components.

The state points for the working fluid are similar to those of the direct-contact cycle. Temperatures for the geo-fluid are also similar; however, because of isolation of geothermal and working fluids the geo-fluid pressure can be much lower than for the direct-contact cycle. For the present analyses geo-fluid pressure was assumed to be 150 psia throughout the cycle (to keep the dissolved nitrogen in solution) and is not included in Table XII. As was done for the triple-condensing, direct-contact cycle, cooling water was bypassed as required to attain consistency with the selected condensing temperatures and condenser pinch points. The amount of bypass can be deduced from the cooling-water flows listed in Table XII.

6.5.2 Power Balance. The power balance for the triple-boiling, triple-condensing, isobutane shelland-tube cycle is shown in Table XIII. Turbine power and parasitics, consisting of working-fluid pumping power and cooling-tower power, are shown in watt-hr/lbm geo-fluid. Under the ground rule of giving credit for geo-fluid discharge pressures in excess of 100 psia, and with the assumption of zero frictional pressure drops, the geo-fluid pumping parasitic loss becomes zero. A small correction to the cooling-tower parasitic loss was included to account for the cooling-water bypass discussed in the preceding paragraph. 
The net plant power for this cycle was estimated to be 4.824 watt-hr/lbm geo-fluid. This value is within $1 \%$ of the net plant power estimated for the corresponding direct-contact cycle having $5^{\circ} \mathrm{F}$ boiler $/$ heater pinch points, power-recovery expanders, and working-fluid recovery components.

6.5.3 Sensitivity of Cycle to Boiler Temperatures. The present effort did not include detailed optimization of boiler temperatures for individual cycles. Boiler temperatures were optimized in Reference 1 for dual-boiling, shell-and-tube cycles; these results were used as a guide for selection of the boiler temperatures used in this study. To obtain a partial check on the proximity of the selected boiler temperatures for the triple-boiling cycles to the optima, two additional cycles were analyzed. These were triple-boiling, single-condensing, isobutane shell-and-tube cycles having condensing temperatures of $104^{\circ} \mathrm{F}$, and with boiler temperatures of $260,210,150^{\circ} \mathrm{F}$, and $250,200,150^{\circ} \mathrm{F}$. The second set of temperatures placed a more nearly equal heat load on the three boilers/heaters.

The results of these calculations are shown in Table XIV. Values of turbine power and working-fluid pumping power are shown in units of watt-hr/lbm geo-fluid. Turbine flows are shown as dimensionless quantities by dividing by the geo-fluid flow.

Three general conclusions were reached from Table XIV. First, flow and power distributions are moderately sensitive to boiler temperatures (even if those temperatures are near optimum), but net plant power is insensitive. Second, boiler temperatures should be selected to result in heat loads that are divided nearly equally between the separate boiler/heater units for components connected as was assumed here. Third, the results did not introduce any evidence indicating that the boiler temperatures selected were sufficiently far from optimum to invalidate comparisons made between cycles in this study. 


\section{OVERALL CONCLUSIONS AND RECOMMENDATIONS}

Specific results and conclusions from the present study are discussed in the Introduction and Summary, Section I-4. Overall conclusions and recommendations are made as follows:

(1) Net plant power (at the same geo-fluid flow) is about the same for a direct-contact isobutane cycle having $5^{\circ} \mathrm{F}$ boiler/heater pinch points, shell-and-tube condensers, and optional power-recovery expanders, as for a comparable shell-and-tube cycle with $10^{\circ} \mathrm{F}$ boiler/heater pinch points.

(2) An improvement of up to $19 \%$ in net plant power, relative to the first Raft River 5-MW dual-boiling pilot plant (at the same geo-fluid flow), can be provided by a triple-boiling, triple-condensing isobutane direct-contact cycle with a working-fluid loss of $30 \times 10^{-6}$ times the geo-fluid flow.

(3) With moderately optimistic assumptions regarding the performance and economics of direct-contact components, a direct-contact binary cycle appears to have the potential for somewhat lower cost electrical energy than does a comparable shell-and-tube cycle.

(4) Work recommended before finalizing a second 5-MW Raft River Pilot Plant includes additional study of heat-rejection systems, and economic assessment and comparisons of the more promising direct-contact and shell-and-tube cycles. 


\section{REFERENCES}

1. I. J. Ingvarsson and S. E. Turner, Working Fluid and Cycle Selection Criteria for Binary Geothermal Power Plants with Resource Temperatures in the Range of $220^{\circ} \mathrm{F}$ to $400^{\circ} \mathrm{F}$, TREE-1108 (April 1977).

2. H. R. Jacobs, R. F. Boehm, A. C. Hansen, Application of Direct Contact Heat Exchangers to Geothermal Power Production Cycles, IDO/1549-8 (January 1978).

3. C. A. Meyer et al, 1967 ASME Steam Tables, New York: ASME, 1967.

4. K. E. Starling, Fluid Thermodynamic Properties for Light Petroleum Systems, Houston, Texas: Gulf Publishing Company, 1973.

5. H. A: Pray, C. E. Schweickert, B. H. Minnich, "Solubility of Hydrogen, Oxygen, Nitrogen, and Helium in Water," Industrial and Engineering Chemistry, 44, 5 (May 1952).

6. D. L. Katz et al, Handbook of Natural Gas Engineering, New York: McGraw-Hill Book Company, Inc., 1959.

7. R. C. Spencer, K. C. Cotton, C. N. Cannon, "A Method for Predicting the Performance of Steam Turbine-Generators," Journal of Engineering for Power, 85, 4 (October 1963).

8. R. Brown, V. Ganapathy, J. Glass, "Design of Air-Cooled Exchangers," Chemical Engineering, 85, 7 (March 1978).

9. R. S. Deeds, THERPP, A Thermodynamics Properties Program, TREE-1081 (May 1977). 
•

• 


\section{APPENDIX A}

\section{FIGURES}




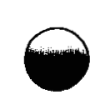

$\theta$ 


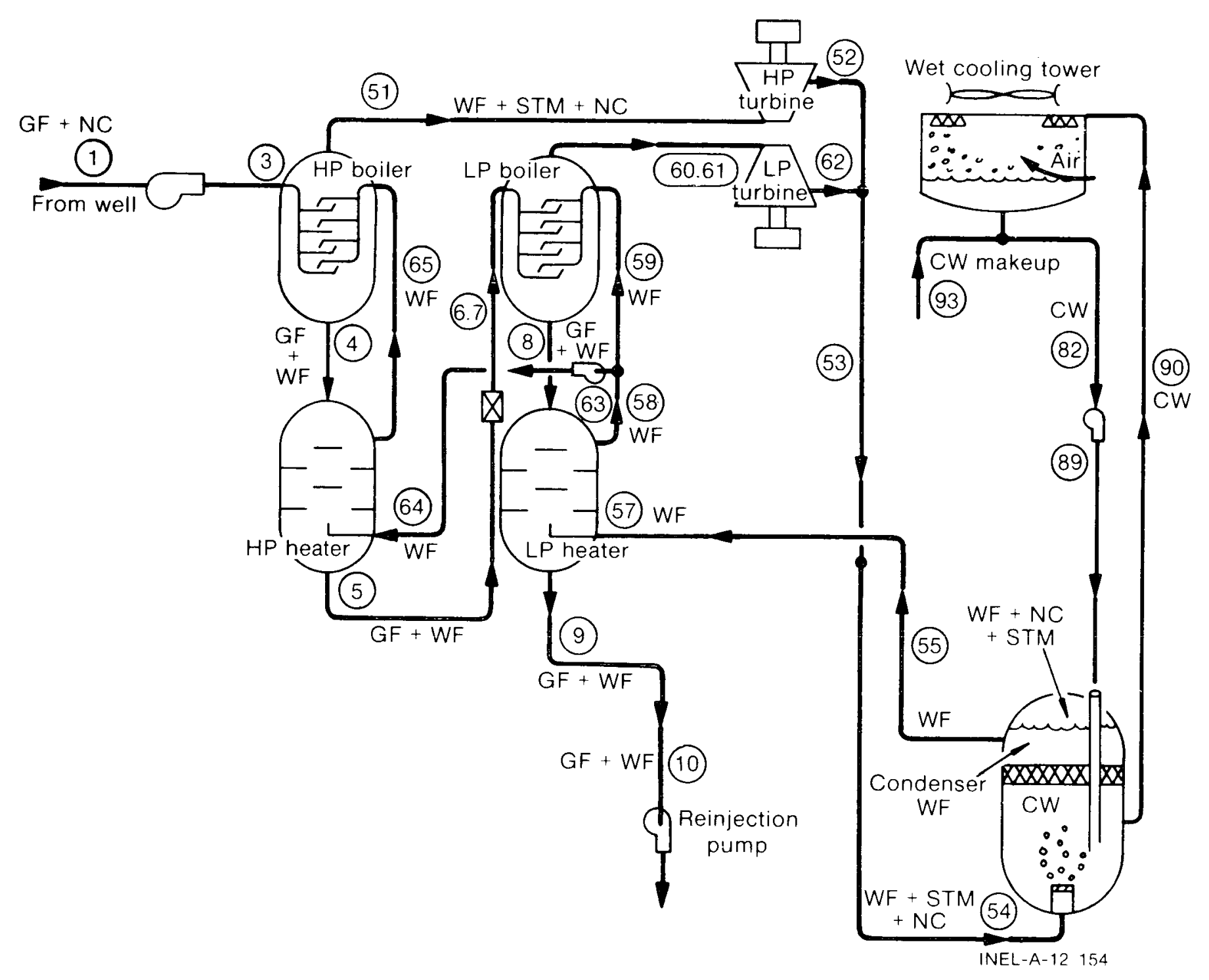

Fig. 1 Dual-boiling, direct-contact geothermal cycle; basic cycle components. 


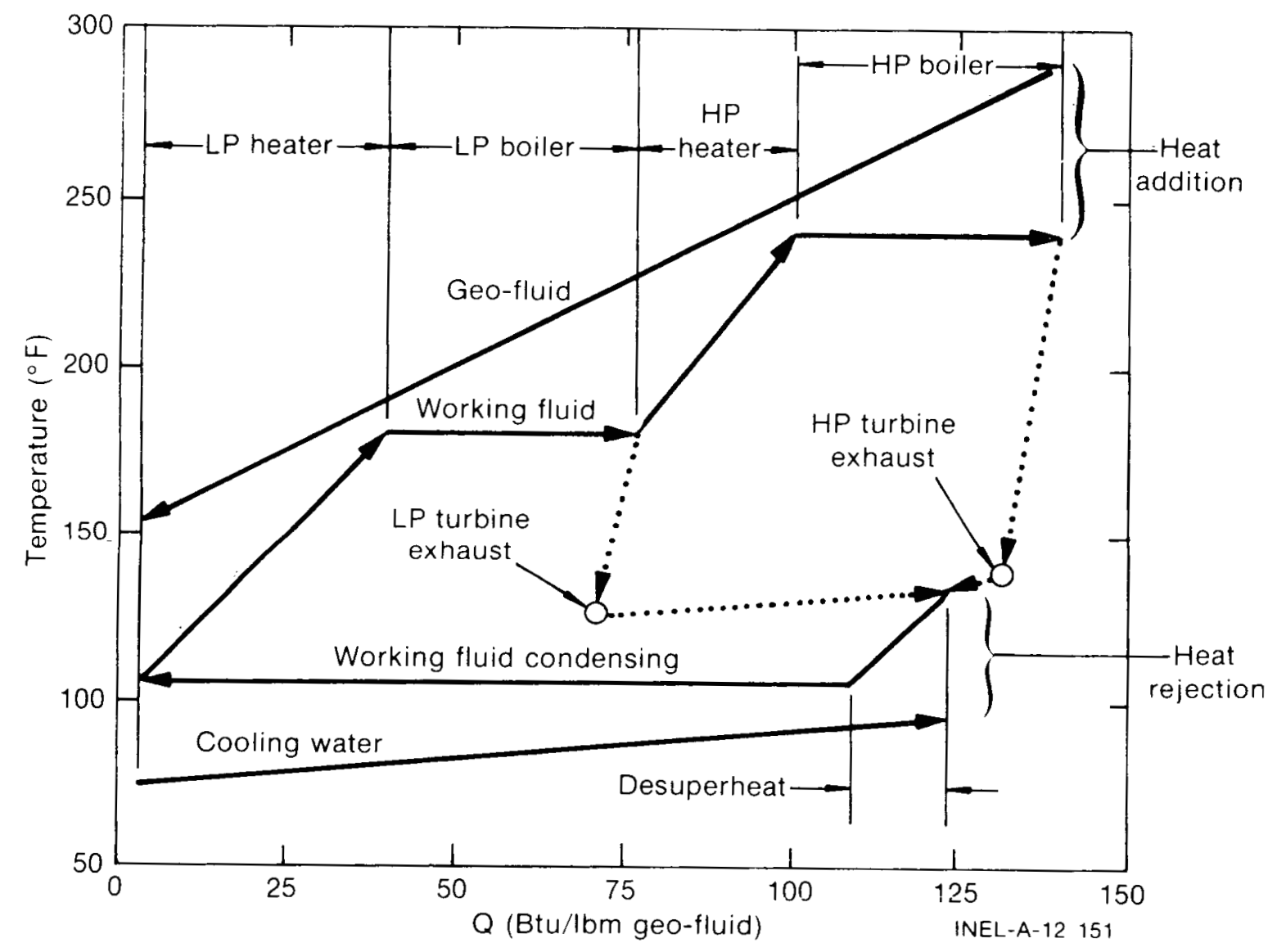

Fig. 2 Temperature - heat exchanged diagram for dual-boiling isobutane cycle. 


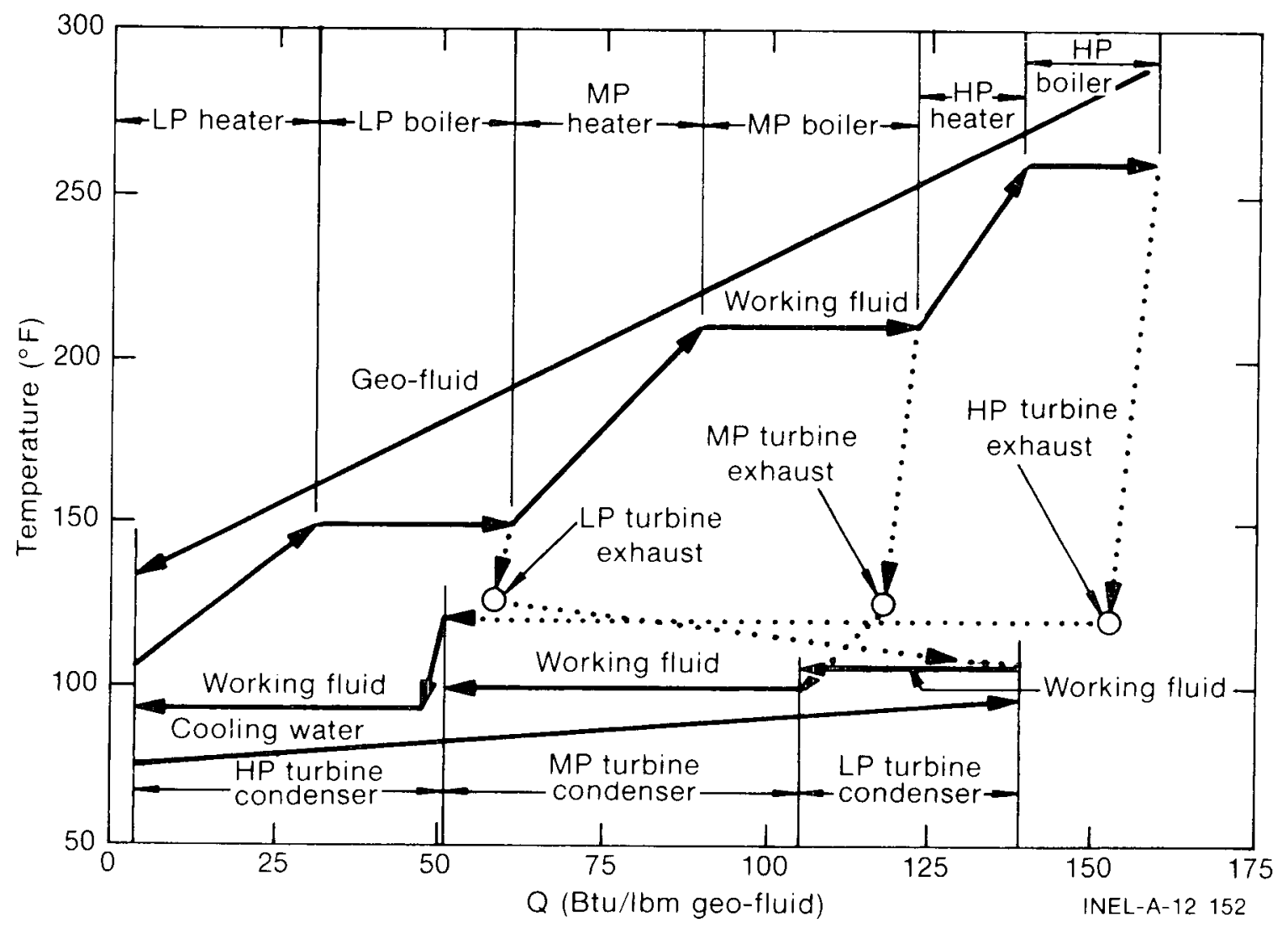

Fig. 3 Temperature - heat exchanged diagram for triple-boiling, triple-condensing isobutane cycle. 


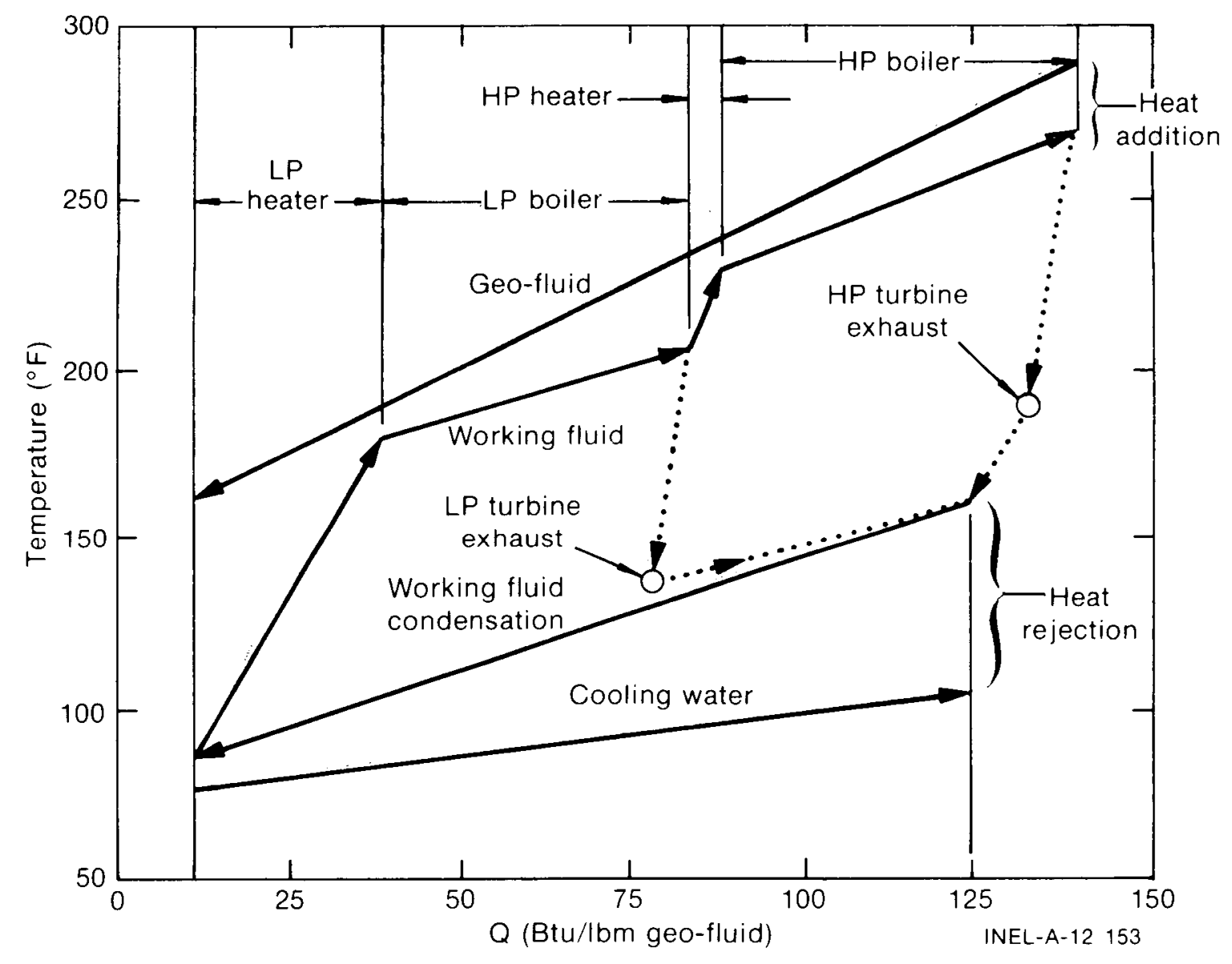

Fig. 4 Temperature - heat exchanged diagram for dual-boiling 0.5 IC4/0.5 IC6 cycle. 


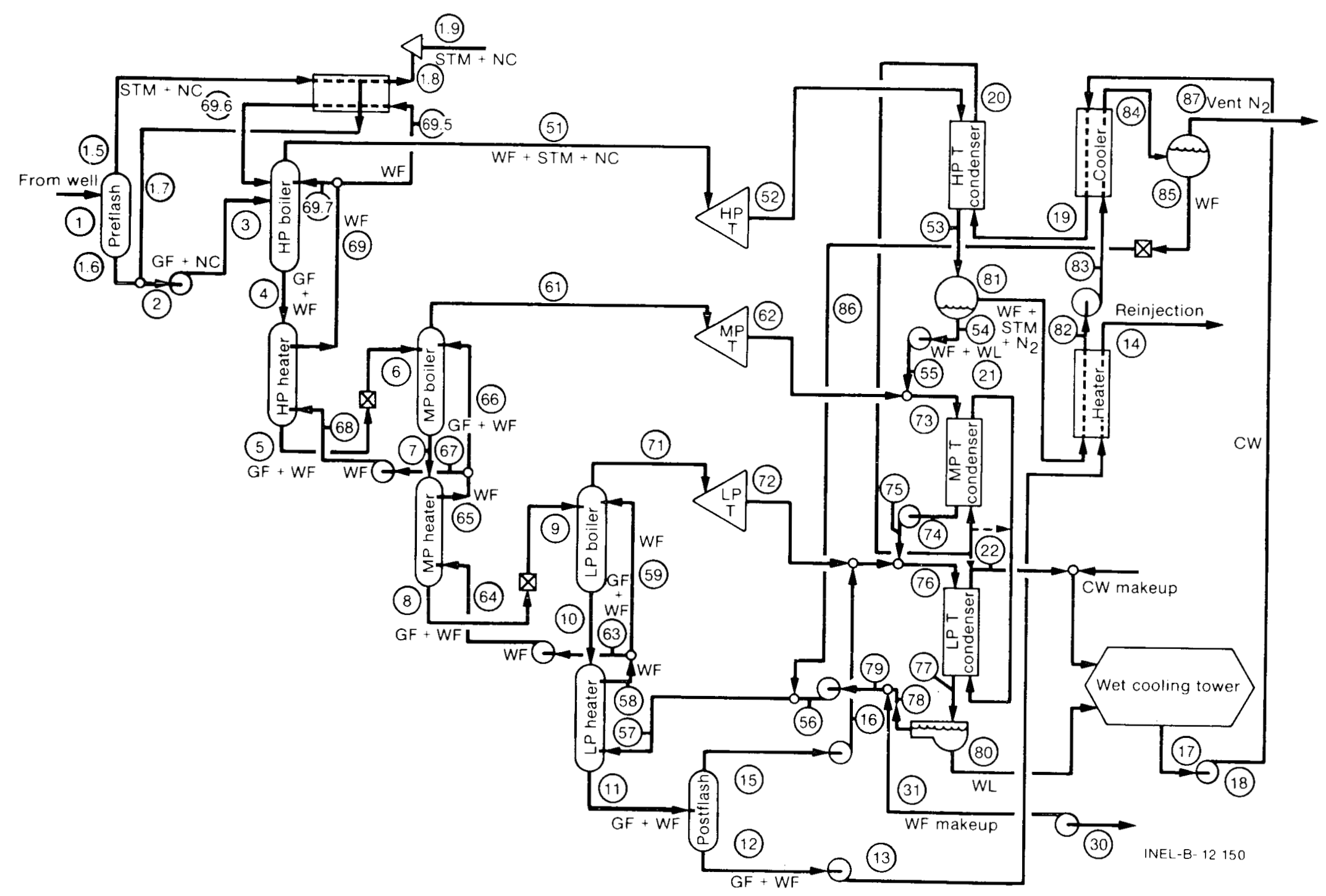

Fig. 5 Triple-boiling, triple-condensing, direct-contact geothermal cycle with working-fluid recovery 


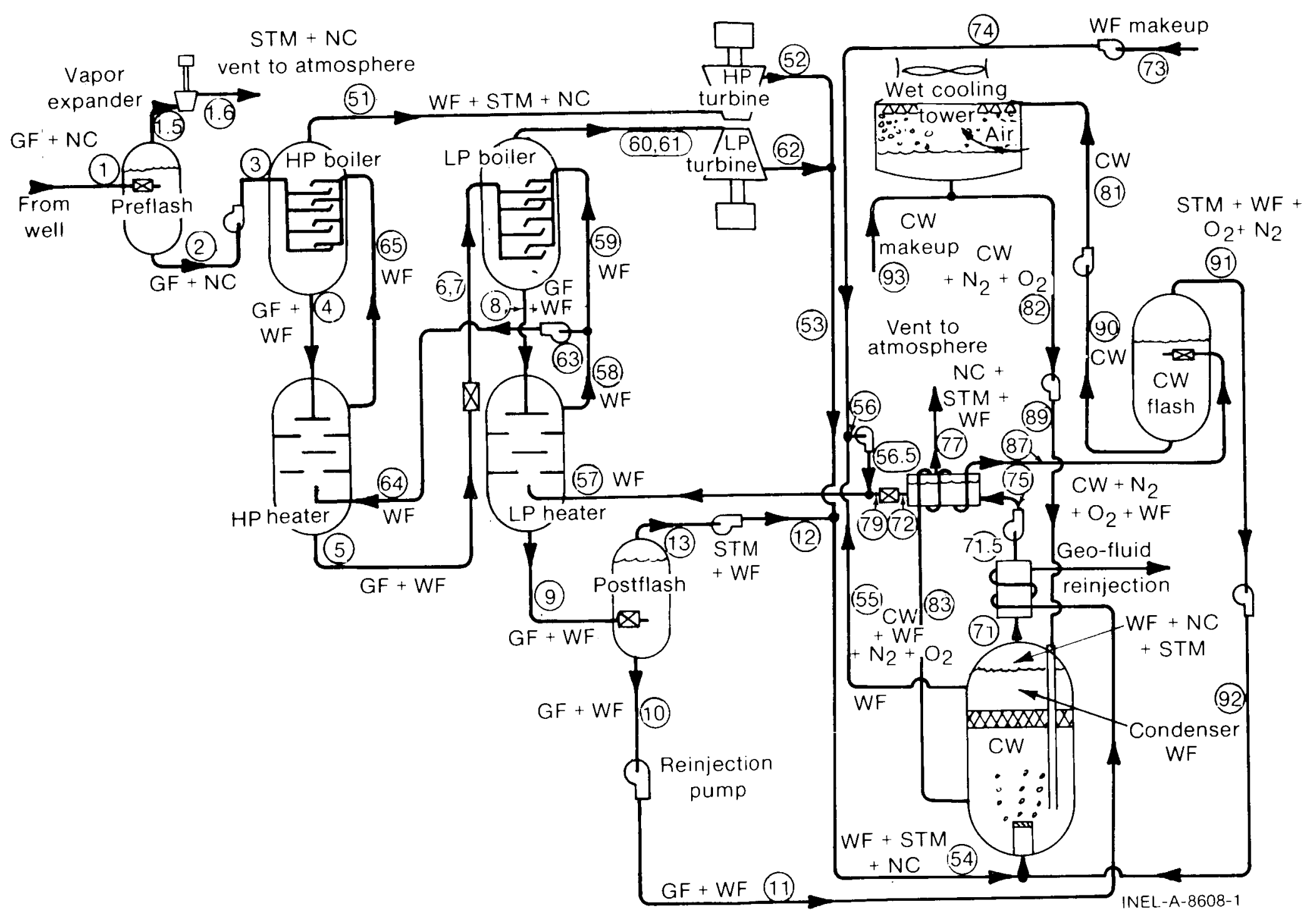

Fig. 6 Dual-boiling, direct-contact cycle with working-fluid recovery. 


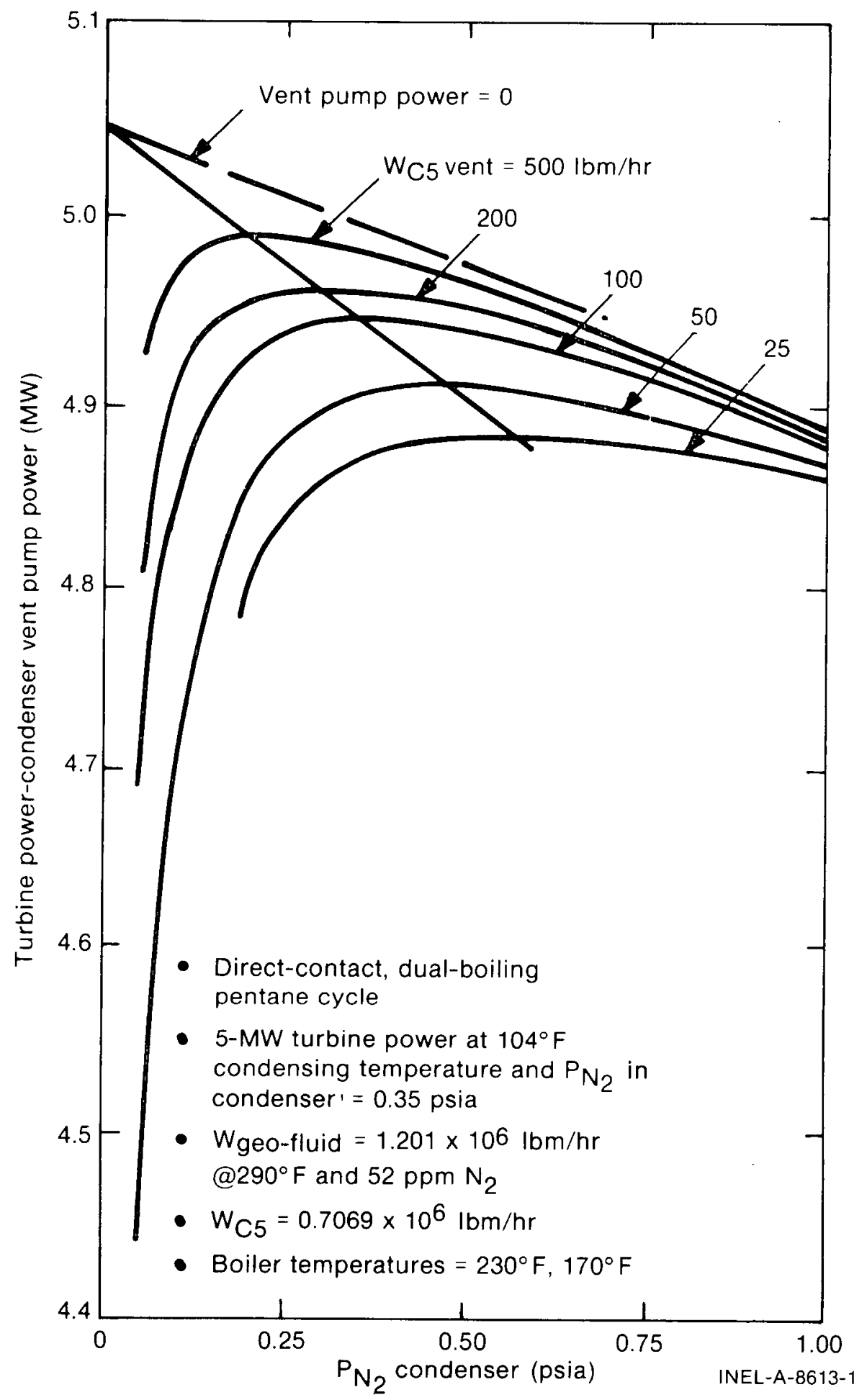

Fig. 7 Effect of $\mathrm{N}_{2}$ partial pressure in condenser on cycle performance; condenser-vent products compressed and cooled. 


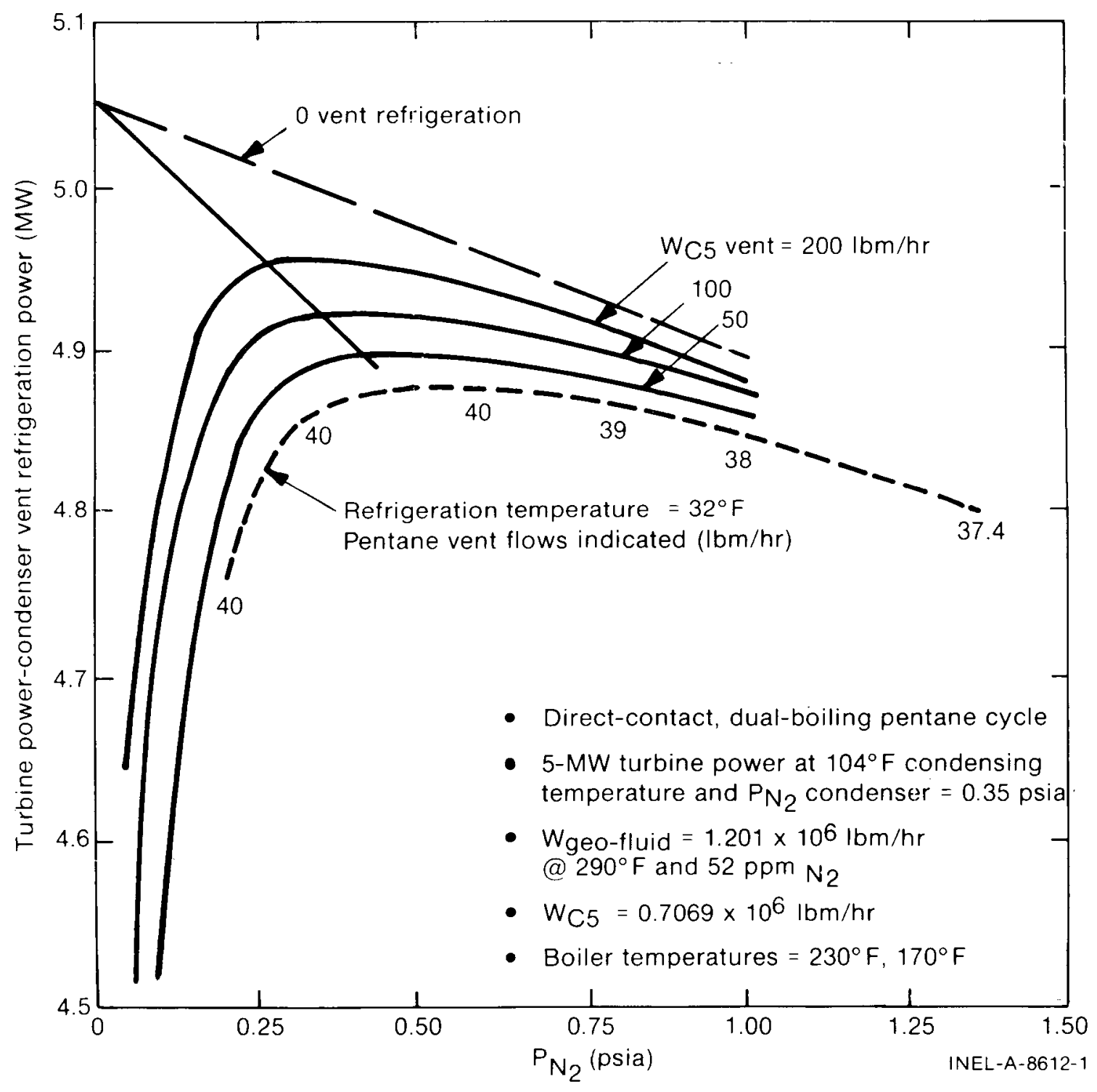

Fig. 8 Effect of $\mathrm{N}_{2}$ partial pressure in condenser on cycle performance; condenser-vent products refrigerated. 


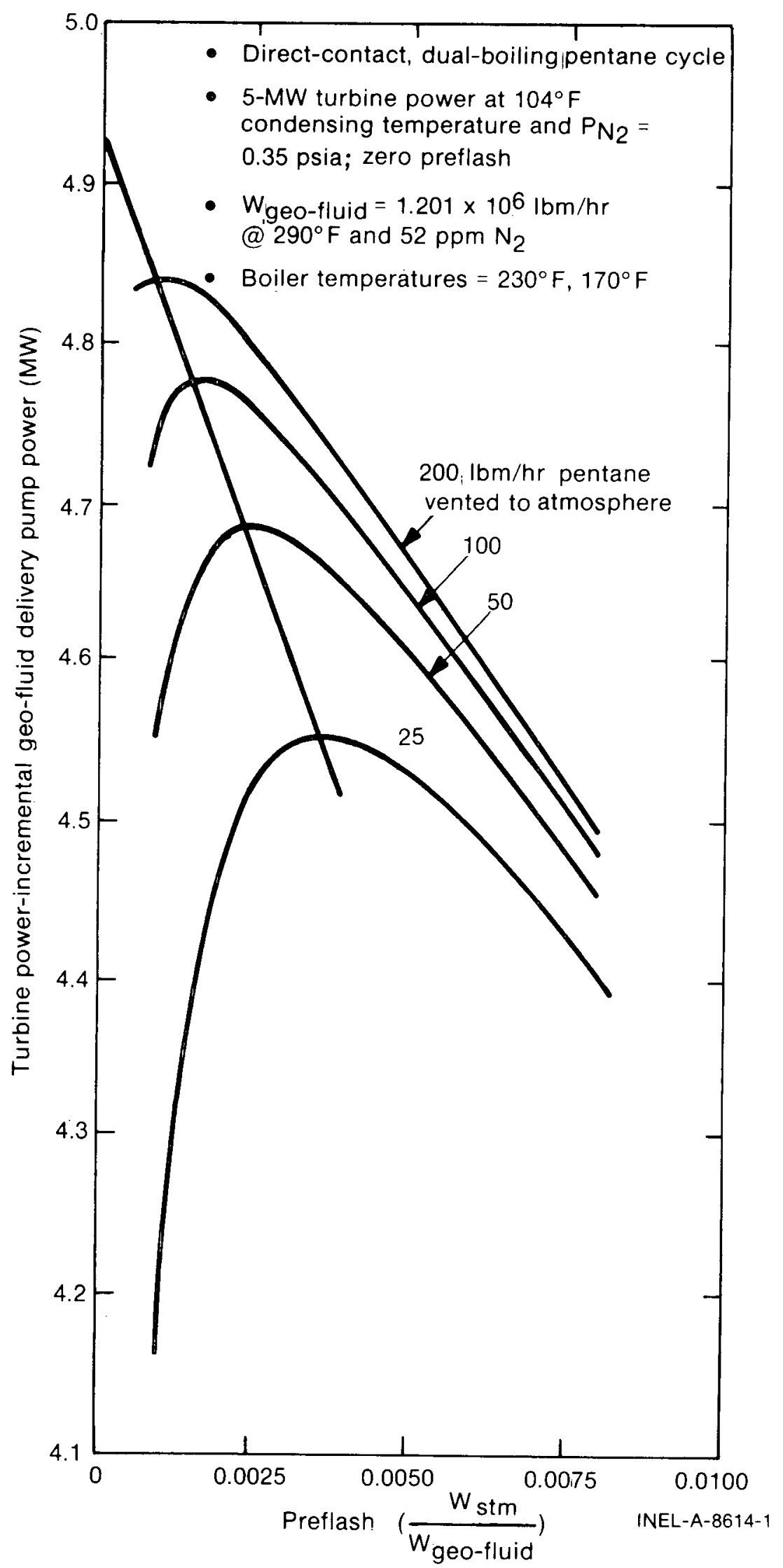

Fig. 9 Effect of preflashing geo-fluid upstream of boilers for direct-contact, dual-boiling pentane cycle. 


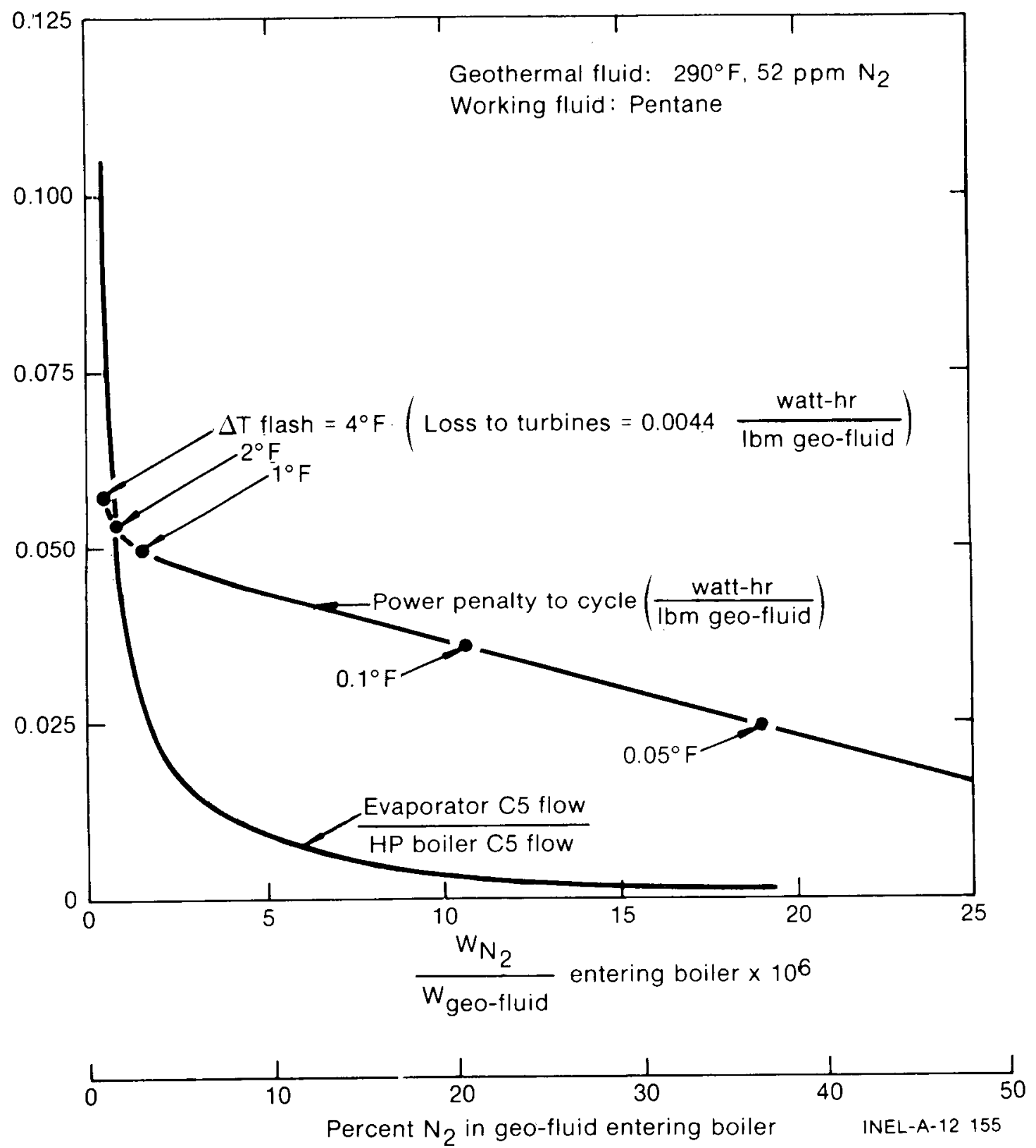

Fig. 10 Effect of preflash with heat-recovery evaporator on dual-boiling, direct-contact cycle. 


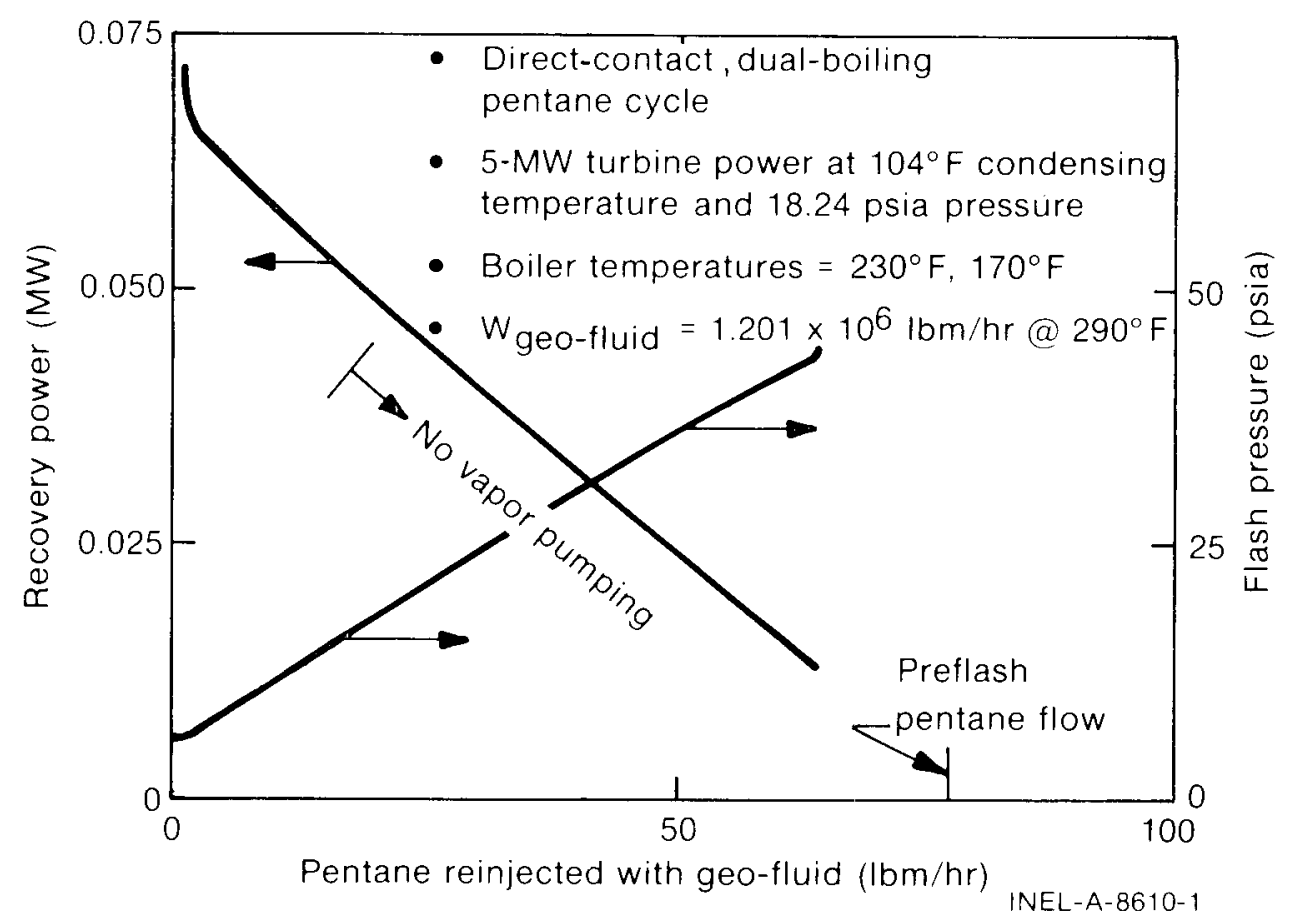

Fig. 11 Power required for recovery of dissolved pentane by geo-fluid flashing process. 
$\theta$ 


\section{APPENDIX B}

TABLES 
.

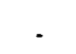

• 
TABLE I

COMPARISONS OF GEOTHERMAL CYCLES USING RAFT RIVER GEO-FLUID

[Direct-Contact Cycles (Working-Fluid Recovery Power not Included)]

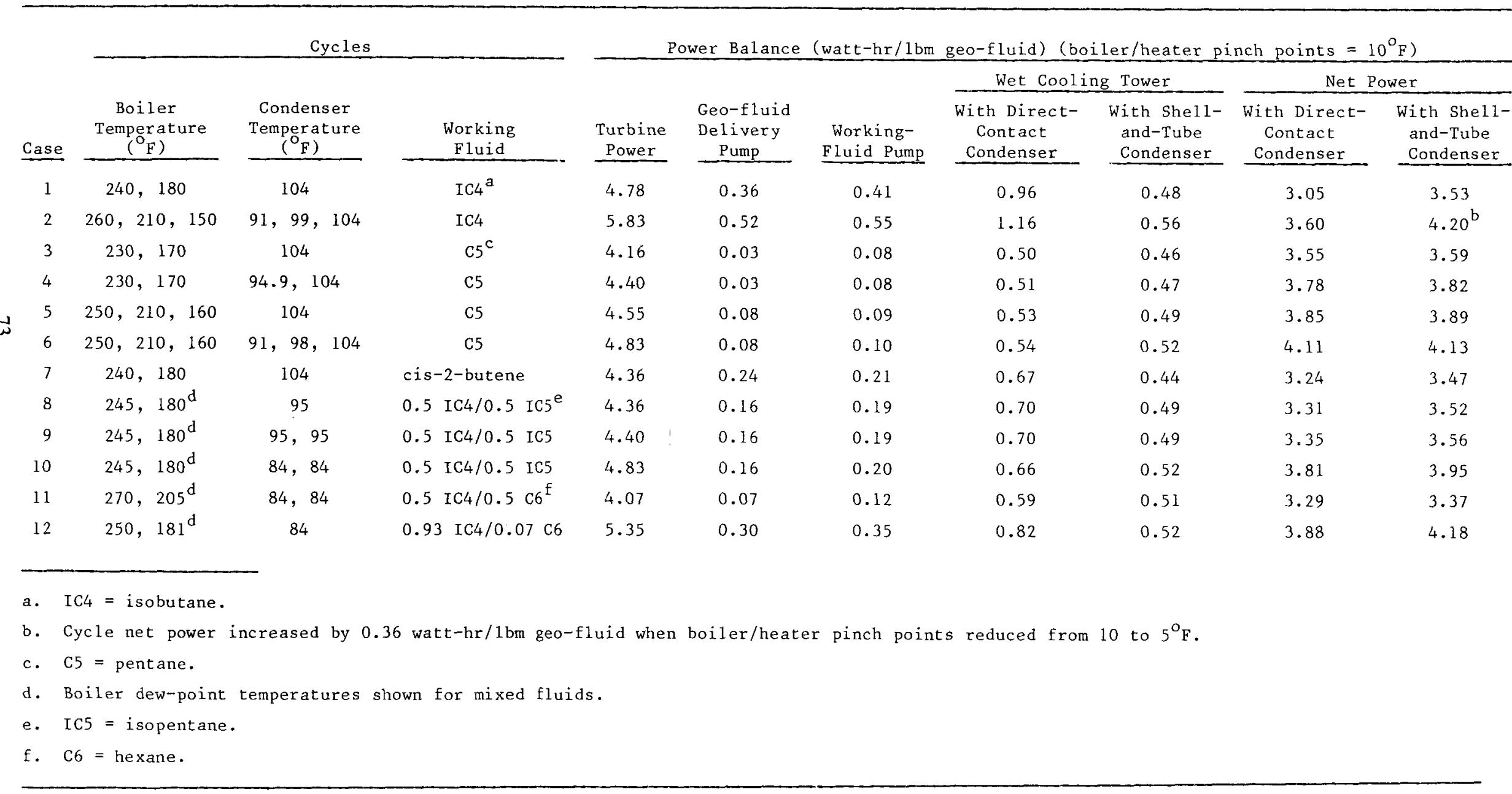


TABLE II

COMPARISON OF GEOTHERMAL CYCLES USING RAFT RIVER GEO-FLUID

(Shell-andi-Tube Cycles)

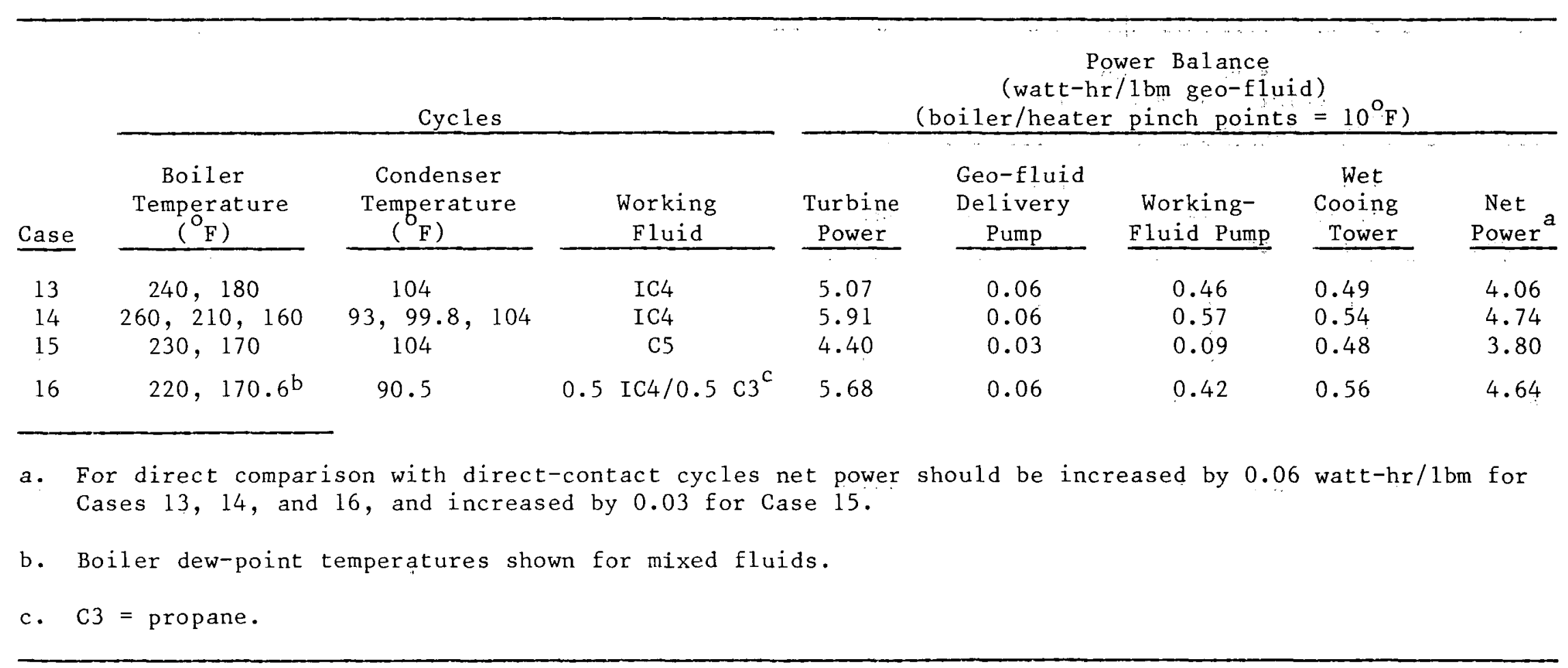




\section{TABLE III}

WORKING-FLUID RECOVERY PARASITICS FOR

TRIPLE-BOILING, ISOBUTANE, DIRECT-CONTACT CYCLE

WITH SHELL-AND-TUBE CONDENSER (RAFT RIVER CONDITIONS)

(Working-Fluid Loss/Geo-Fluid Flow $=30 \times 10^{-6}$ )

\begin{tabular}{|c|c|c|}
\hline Recovery Item & $\begin{array}{c}\text { Penalty to Cycle } \\
\text { (Watt-hr/lbm geo-fluid) }\end{array}$ & $\begin{array}{l}\text { Percent of } \\
\text { Net Power }\end{array}$ \\
\hline Preflash geo-fluid & 0.05 & 1.1 \\
\hline Preflash vapor expander $(\eta=0.7)$ & -0.004 & -0.1 \\
\hline Preflash geo-fluid expander $(\eta=0.6)$ & -0.02 & -0.4 \\
\hline $\begin{array}{l}\text { Geo-fluid reinjection following } \\
\text { postflash ( } 7.35 \text { to } 100 \text { psia) }\end{array}$ & $0.10+0.06^{a}=0.16$ & 3.6 \\
\hline Postflash recovery expander $(\eta=0.6)$ & -0.07 & -1.6 \\
\hline Postflash vapor compressor & 0.004 & 0.1 \\
\hline Condenser-vent compressor & 0 & 0 \\
\hline Total penalty & 0.12 & 2.7 \\
\hline \multicolumn{3}{|c|}{$\begin{array}{l}\text { a. Without postflash, the cycle could have taken credit for the increment } \\
\text { in pressure by which the low-pressure boiler exceeds } 100 \text { psia. }\end{array}$} \\
\hline
\end{tabular}


TABLE IV

POWER-RECOVERY EXPANDERS FOR DIRECT-CONTACT CYCLES

\begin{tabular}{|c|c|c|c|c|c|c|}
\hline \multirow[b]{2}{*}{ Cycle } & \multicolumn{6}{|c|}{$\begin{array}{c}\text { Power Balance } \\
\text { (watt-hr/lbm geo-fluid) }\end{array}$} \\
\hline & $\begin{array}{l}\text { Turbine } \\
\text { Power }\end{array}$ & Parasitics & $\begin{array}{c}\text { Expander } \\
\text { Power }\end{array}$ & $\begin{array}{l}\text { Net Power } \\
\text { Without } \\
\text { Expander }\end{array}$ & $\begin{array}{l}\text { Net Power } \\
\quad \text { With } \\
\text { Expander }\end{array}$ & $\begin{array}{l}\text { Net } \\
\text { Power } \\
\text { Gain } \\
(\%)\end{array}$ \\
\hline Dual-boiling Isobutane & 4.78 & 1.25 & 0.12 & 3.53 & 3.65 & 3.4 \\
\hline Dua1-boiling Pentane & 4.16 & 0.57 & 0.05 & 3.59 & 3.64 & 1.4 \\
\hline Triple-boiling Isobutane & 5.83 & 1.63 & $\begin{aligned} 0.15 & +0.11 \\
& =0.26\end{aligned}$ & 4.20 & 4.46 & 6.2 \\
\hline
\end{tabular}

a. Working-fluid recovery power, and effects of expanders on cycle not included in this table. 
TABLE V

HEAT-REJECTION PARASITICS

\begin{tabular}{|c|c|c|c|c|}
\hline $\begin{array}{c}\text { Heat-Rejection System } \\
(65 \mathrm{~F} \text { Wet Bulb })\end{array}$ & $\begin{array}{l}\text { Basic } \\
\text { Parasitic } \\
\text { (watt-hr/lbm } \\
\text { geo-fluid) } \\
\end{array}$ & $\begin{array}{l}\Delta \text { Turbine } \\
\quad \text { Power } \\
\text { (watt-hr/lbm } \\
\text { geo-fluid) } \\
\end{array}$ & $\begin{array}{l}\Delta \text { Working- } \\
\text { Fluid } \\
\text { Recovery } \\
\text { Power } \\
\end{array}$ & $\begin{array}{r}\text { Equivalent } \\
\text { Parasitic } \\
\text { (watt-hr/lbm } \\
\text { geo-fluid) } \\
\end{array}$ \\
\hline $\begin{array}{l}\text { Wet tower, shel1-and-tube } \\
\text { condenser, } 90^{\circ} \text { pinch, } \\
\mathrm{T}_{\mathrm{C}}=104^{\circ} \mathrm{F} \text {, isobutane } \\
\text { (reference case) }\end{array}$ & 0.45 & 0 & 0 & 0.45 \\
\hline $\begin{array}{l}\text { Wet tower, direct-contact } \\
\text { condenser, } 5^{\circ} \mathrm{F} \text { pinch, } \\
{ }^{C}=100^{\circ} \mathrm{F} \text {, pentane }\end{array}$ & 0.44 & +0.21 & $+0.26^{b}$ & 0.49 \\
\hline $\begin{array}{l}\text { Wet tower, direct-contact } \\
\text { condenser, } 5^{\circ} \mathrm{F} \text { pinch, } \\
{ }^{\mathrm{T}} \mathrm{C}=100^{\circ} \mathrm{F}, \text { is obutane }\end{array}$ & 0.93 & +0.19 & $+0.29^{b}$ & 1.03 \\
\hline $\begin{array}{l}\text { Spray-augmented dry tower, } \\
\text { direct-contact condenser, } \\
5^{\circ} \mathrm{F} \text { pinch, } \mathrm{T}_{\mathrm{C}}=100^{\circ} \mathrm{F} \\
\text { isobutane }\end{array}$ & 1.17 & +0.19 & 0 & 0.98 \\
\hline
\end{tabular}


TABLE V (continued)

\begin{tabular}{|c|c|c|c|c|}
\hline $\begin{array}{c}\text { Heat-Rejection System } \\
\left(65^{\circ} \mathrm{F} \text { Wet Bulb }\right)\end{array}$ & $\begin{array}{l}\quad \text { Basic } \\
\text { Parasitic } \\
\text { (watt-hr/lbm } \\
\text { (geo-fluid) } \\
\end{array}$ & 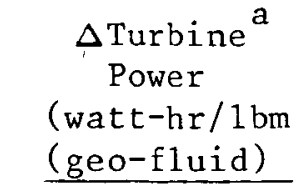 & $\begin{array}{l}\Delta \text { Working-a } \\
\text { Fluid } \\
\text { Recovery } \\
\text { Power } \\
\end{array}$ & $\begin{array}{r}\text { Equivalent } \\
\text { Parasitic } \\
\text { (watt-hr/lbm } \\
\text { geo-fluid) } \\
\end{array}$ \\
\hline $\begin{array}{l}\text { Evaporative condenser } \\
\mathrm{T}_{\mathrm{C}}=104^{\circ} \mathrm{F}, \text { isobutane }\end{array}$ & 0.41 & 0 & 0 & 0.41 \\
\hline $\begin{array}{l}\text { Evaporative condenser } \\
\mathrm{T}_{\mathrm{C}}=84^{\circ} \mathrm{F} \text {, isobutane }\end{array}$ & 0.95 & +0.90 & 0 & 0.05 \\
\hline a. Relative to reference & & & & \\
\hline b. Working-fluid recover & increments prol & verestimated. & & \\
\hline
\end{tabular}




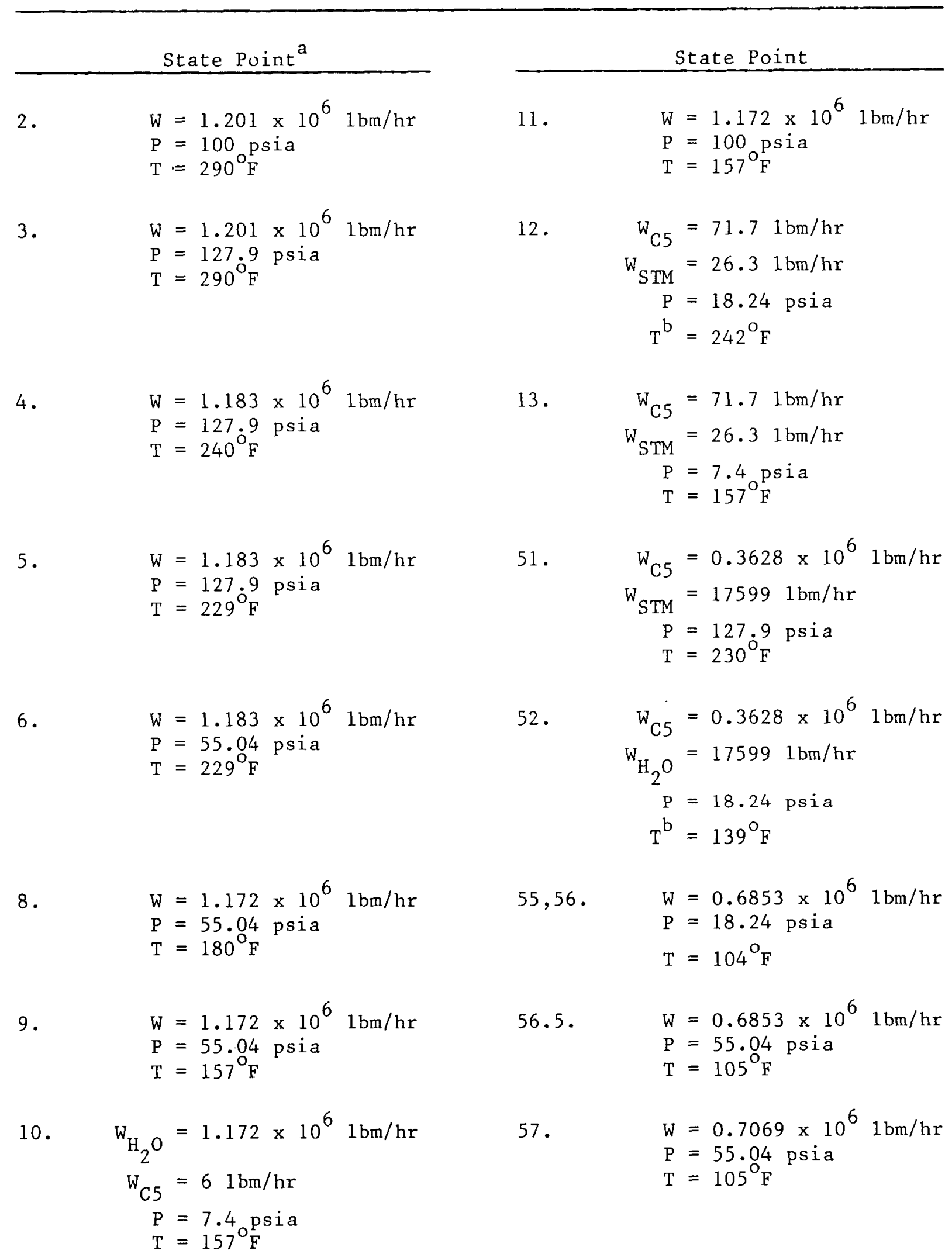


State Point ${ }^{a}$

58. $\quad W=0.7069 \times 10^{6} 1 \mathrm{bm} / \mathrm{hr}$

$\mathrm{P}=55.04$ psia

$\mathrm{T}=170^{\circ} \mathrm{F}$

59. $\mathrm{W}=0.3442 \times 10^{6} 1 \mathrm{bm} / \mathrm{hr}$

$\mathrm{P}=55.04$ psia

$\mathrm{T}=170^{\circ} \mathrm{F}$

60. $\mathrm{W}_{\mathrm{C} 5}=0.3442 \times 10^{6} \mathrm{1bm} / \mathrm{hr}$

$$
\begin{aligned}
\mathrm{W}_{\mathrm{STM}} & =10512 \mathrm{lbm} / \mathrm{hr} \\
\mathrm{P} & =55.04 \mathrm{psia} \\
\mathrm{T} & =170^{\circ} \mathrm{F}
\end{aligned}
$$

62.

$$
\begin{aligned}
\mathrm{W}_{\mathrm{C} 5} & =0.3442 \times 10^{6} 1 \mathrm{bm} / \mathrm{hr} \\
\mathrm{W}_{\mathrm{H}_{2} \mathrm{O}} & =10512 \times 1 \mathrm{bm} / \mathrm{hr} \\
\mathrm{P} & =18.241 \mathrm{bm} / \mathrm{hr} \\
\mathrm{T}^{\mathrm{b}} & =124^{\circ} \mathrm{F}
\end{aligned}
$$

63.

$$
\begin{aligned}
& W=0.3628 \times 10^{6} 1 \mathrm{bm} / \mathrm{hr} \\
& P=55.04 \text { psia } \\
& T=170 \mathrm{~F}
\end{aligned}
$$

State Point

71.

$$
\begin{aligned}
\mathrm{W}_{\mathrm{C} 5} & =218001 \mathrm{bm} / \mathrm{hr} \\
\mathrm{W}_{\mathrm{STM}} & =3201 \mathrm{bm} / \mathrm{hr} \\
\mathrm{W}_{\mathrm{N}_{2}} & =1301 \mathrm{bm} / \mathrm{hr} \\
\mathrm{W}_{\mathrm{O}_{2}} & =401 \mathrm{bm} / \mathrm{hr} \\
\mathrm{P} & =18.24 \mathrm{psia} \\
\mathrm{T} & =104^{\mathrm{O}} \mathrm{F}
\end{aligned}
$$

72.

$$
\begin{aligned}
& \mathrm{W}=22 \quad 100 \quad 1 \mathrm{bm} / \mathrm{hr} \\
& \mathrm{P}=128 \mathrm{psia} \\
& \mathrm{T}=105 \mathrm{~F}
\end{aligned}
$$

73.

$$
\mathrm{W}=36 \mathrm{lbm} / \mathrm{hr}
$$$$
\mathrm{P}=7 \mathrm{psia}
$$$$
\mathrm{T}=60^{\circ} \mathrm{F}
$$

75.

$$
\begin{aligned}
& \mathrm{W}=22300 \quad \mathrm{bm} / \mathrm{hr} \\
& \mathrm{P}=128 \mathrm{psia} \\
& \mathrm{T}=220 \mathrm{~F}
\end{aligned}
$$

77 .

$$
\begin{aligned}
\mathrm{W}_{\mathrm{C} 5} & =251 \mathrm{bm} / \mathrm{hr} \\
\mathrm{W}_{\mathrm{N}_{2}} & =1301 \mathrm{bm} / \mathrm{hr} \\
\mathrm{W}_{\mathrm{O}_{2}} & =401 \mathrm{bm} / \mathrm{hr} \\
\mathrm{W}_{\mathrm{STM}} & =11 \mathrm{bm} / \mathrm{hr} \\
\mathrm{P} & =128 \mathrm{psia} \\
\mathrm{T} & =105^{\circ} \mathrm{F} \\
\mathrm{W} & =216001 \mathrm{bm} / \mathrm{hr} \\
\mathrm{P} & =55.04 \mathrm{psia} \\
\mathrm{T} & =106^{\circ} \mathrm{F} \\
\mathrm{W}_{\mathrm{H}_{2} \mathrm{O}} & =6.773 \mathrm{x} 10^{6} 1 \mathrm{bm} \\
\mathrm{W}_{\mathrm{C} 5} & =51 \mathrm{bm} / \mathrm{hr} \\
\mathrm{P} & =85 . \mathrm{psia} \\
\mathrm{T} & =96^{\circ} \mathrm{F}
\end{aligned}
$$

79.$$
\text { 81. } \mathrm{W}_{\mathrm{H}_{2} \mathrm{O}}=6.773 \times 10^{6} 1 \mathrm{bm} / \mathrm{hr}
$$ 


\section{State Point ${ }^{a}$}

82.

$$
\begin{aligned}
& \mathrm{W}=6.773 \times 10^{6} \mathrm{lbm} / \mathrm{hr} \\
& \mathrm{P}=1.4 .7 \text { psia } \\
& \mathrm{T}=75^{\circ} \mathrm{F}
\end{aligned}
$$

83.

$$
\begin{aligned}
& W=6.773 \times 10^{6} 1 \mathrm{bm} / \mathrm{hr} \\
& \mathrm{P}=18.24 \mathrm{psia} \\
& \mathrm{T}=95^{\circ} \mathrm{F}
\end{aligned}
$$

\section{State Point}

90 .

$$
W=6.773 \times 10^{6} \mathrm{lbm} / \mathrm{hr}
$$$$
\mathrm{P}=1.35 \mathrm{psia}
$$$$
\mathrm{T}=96^{\circ} \mathrm{F}
$$

91.

$$
\begin{aligned}
\mathrm{W}_{\mathrm{STM}} & =2021 \mathrm{bm} / \mathrm{hr} \\
\mathrm{W}_{\mathrm{N}_{2}} & =661 \mathrm{bm} / \mathrm{hr} \\
\mathrm{W}_{\mathrm{O}_{2}} & =361 \mathrm{bm} / \mathrm{hr} \\
\mathrm{W}_{\mathrm{C} 5} & =2901 \mathrm{bm} / \mathrm{hr} \\
\mathrm{P} & =1.35 \mathrm{psia} \\
\mathrm{T} & =96^{\circ} \mathrm{F}
\end{aligned}
$$

92.

$$
\begin{aligned}
\mathrm{W} & =5941 \mathrm{bm} / \mathrm{hr} \\
\mathrm{P} & =18.24 \mathrm{psia} \\
\mathrm{T}^{\mathrm{b}} & =375^{\circ} \mathrm{E}
\end{aligned}
$$

89.

$$
\begin{aligned}
& \mathrm{W}=6.773 \times 10^{6} \mathrm{lbm} / \mathrm{hr} \\
& \mathrm{P}=18.24 \mathrm{psia} \\
& \mathrm{T}=75_{\mathrm{F}}
\end{aligned}
$$

93.

a. State point numbers correspond to locations shown on Figure 6 .

b. Temperatures correspond to isentropic process; actual temperatures are higher. 
POWER BALANCE FOR DIRECT-CONTACT, DUAL-BOILING PENTANE CYCLE

\begin{tabular}{|c|c|c|}
\hline & $\begin{array}{l}\text { Power } \\
\text { (MW) }\end{array}$ & $\begin{array}{c}\text { Penalties Assignable } \\
\text { to Direct-Contact Cycle } \\
\text { (MW) }\end{array}$ \\
\hline $\begin{array}{l}\text { Turbines at } \mathrm{P}_{\mathrm{N}_{2}} \text { Condenser } \\
=0.35 \mathrm{psia}\end{array}$ & $3.35+1.65=5.00$ & 0 \\
\hline $\begin{array}{l}\text { Turbines at } \mathrm{P}_{\mathrm{N}_{2}} \text { Condenser } \\
\quad=0\end{array}$ & 5.056 & -- \\
\hline $\begin{array}{l}\text { Geo-fluid pressure-drop } \\
\text { expander }(\eta=0.8)\end{array}$ & 0.056 & -- \\
\hline $\begin{array}{l}\text { Post flash geo-fluid expander } \\
\qquad(\eta=0.6)\end{array}$ & 0.030 & -- \\
\hline $\begin{array}{l}\text { Cooling-water flash expander } \\
\qquad(\eta=0.6)\end{array}$ & 0.061 & -- \\
\hline $\begin{array}{l}\text { Incremental power due to } \\
-5^{\circ} \text { change in pinch } \\
\text { points in boilers/heaters }\end{array}$ & 0.280 & -- \\
\hline $\begin{array}{l}\text { Incremental power due to } \\
-4^{\circ} \text { change in } \\
\text { condenser pinch point }\end{array}$ & 0.256 & -- \\
\hline
\end{tabular}


TABLE VII (continued)

\begin{tabular}{|c|c|c|}
\hline & $\begin{array}{l}\text { Parasitic } \\
\text { Losses } \\
(\mathrm{MW}) \\
\end{array}$ & $\begin{array}{c}\text { Penalties Assignable } \\
\text { to Direct-Contact Cycle } \\
(\mathrm{MW})\end{array}$ \\
\hline Basic wet cooling tower & 0.523 & $\sim 0$ \\
\hline High-pressure feed pump & 0.051 & 0.010 \\
\hline Low-pressure feed pump & 0.047 & 0.006 \\
\hline Geo-fluid delivery pump & 0.038 & 0.011 \\
\hline Geo-fluid reinjection pump & 0.120 & 0.146 \\
\hline $\begin{array}{l}\text { Geo-fluid post flash vapor } \\
\text { pump }\end{array}$ & 0.002 & 0.002 \\
\hline Condenser-vent pump & 0.100 & 0.100 \\
\hline $\begin{array}{l}\text { Turbine loss due to optimum } \\
{ }^{\mathrm{P}} \mathrm{N}_{2} \text { Condenser }\end{array}$ & 0.051 & 0.051 \\
\hline $\begin{array}{l}\text { Condenser pump increment } \\
\text { due to CW flash }\end{array}$ & 0.163 & 0.163 \\
\hline Cooling-water boost pump & 0.125 & 0.125 \\
\hline $\begin{array}{l}\text { Cooling-water flash vapor } \\
\text { pump }\end{array}$ & 0.024 & 0.024 \\
\hline
\end{tabular}


STATE POINTS FOR DIRECT-CONTACT, DUAL-BOILING ISOBUTANE CYCLE

State Point ${ }^{a}$

2. $\mathrm{W}=1.055 \times 10^{6} \mathrm{1bm} / \mathrm{hr}$

$\mathrm{P}=100$ psia

$\mathrm{T}=290^{\circ} \mathrm{F}$

3. $\quad \mathrm{W}=1.055 \times 10^{6} 1 \mathrm{bm} / \mathrm{hr}$

$P=406.5$ psia

$\mathrm{T}=291^{\circ} \mathrm{F}$

4. $\mathrm{W}=1.044 \times 10^{6} 1 \mathrm{bm} / \mathrm{hr}$

$\mathrm{P}=406.5$ psia

$\mathrm{T}=250^{\circ} \mathrm{F}$

5.

$\mathrm{W}=1.044 \times 10^{6} \mathrm{lbm} / \mathrm{hr}$

$\mathrm{P}=406.5$ psia

$\mathrm{T}=228^{\circ} \mathrm{F}$

6. $\mathrm{W}=1.044 \times 10^{6} 1 \mathrm{bm} / \mathrm{hr}$

$\mathrm{P}=210.6 \mathrm{psia}$

$\mathrm{T}=229^{\circ} \mathrm{F}$

8.

$\mathrm{W}=1.040 \times 10^{6} \mathrm{1bm} / \mathrm{hr}$

$\mathrm{P}=210.6 \mathrm{psia}$

$\mathrm{T}=190^{\circ} \mathrm{F}$

9.

\section{State Point}

10 .

$$
\begin{aligned}
\mathrm{W}_{\mathrm{H}_{2} \mathrm{O}} & =1.040 \times 10^{6} \mathrm{lbm} / \mathrm{hr} \\
\mathrm{W}_{\mathrm{C} 4} & =61 \mathrm{bm} / \mathrm{hr} \\
\mathrm{P} & =9.5 \mathrm{psia} \\
\mathrm{T} & =152 \mathrm{O}
\end{aligned}
$$

11 .

$$
\begin{aligned}
& \mathrm{W}=1.040 \times 10^{6} \mathrm{lbm} / \mathrm{hr} \\
& \mathrm{P}=100 \mathrm{psia} \\
& \mathrm{T}=152^{\circ} \mathrm{F}
\end{aligned}
$$

12.

$$
\begin{aligned}
\mathrm{W}_{\mathrm{C} 4} & =209 \mathrm{lbm} / \mathrm{hr} \\
\mathrm{W}_{\mathrm{STM}} & =45 \mathrm{lbm} / \mathrm{hr} \\
\mathrm{P} & =79.78 \mathrm{psia} \\
\mathrm{T}^{\mathrm{b}} & =319^{\circ} \mathrm{F}
\end{aligned}
$$

13.

$$
\begin{aligned}
\mathrm{W}_{\mathrm{C} 4} & =2091 \mathrm{bm} / \mathrm{hr} \\
\mathrm{W}_{\mathrm{STM}} & =451 \mathrm{bm} / \mathrm{hr} \\
\mathrm{P} & =9.5 \mathrm{psia} \\
\mathrm{T} & =152^{\circ} \mathrm{F}
\end{aligned}
$$

51. $\mathrm{W}_{\mathrm{C} 4}=0.4738 \times 10^{6} 1 \mathrm{bm} / \mathrm{hr}$

$$
\begin{aligned}
\mathrm{W}_{\mathrm{STM}} & =9623 \mathrm{lbm} / \mathrm{hr} \\
\mathrm{P} & =406 \cdot 5 \mathrm{psia} \\
\mathrm{T} & =240^{\circ} \mathrm{F}
\end{aligned}
$$

52. $\mathrm{W}_{\mathrm{C} 4}=0.4738 \times 10^{6} \mathrm{1bm} / \mathrm{hr}$

$$
\begin{aligned}
\mathrm{W}_{\mathrm{H}_{2} \mathrm{O}} & =9623 \mathrm{lbm} / \mathrm{hr} \\
\mathrm{P} & =79.78 \text { psia } \\
\mathrm{T}^{\mathrm{b}} & =139^{\circ} \mathrm{F}
\end{aligned}
$$

$$
\text { 55,56. } \begin{aligned}
\mathrm{W} & =0.0811 \times 10^{6} \mathrm{1bm} / \mathrm{hr} \\
\mathrm{P} & =79.78 \mathrm{psia} \\
\mathrm{T}^{\mathrm{b}} & =104^{\circ} \mathrm{F}
\end{aligned}
$$




\begin{tabular}{|c|c|c|c|c|}
\hline \multicolumn{3}{|c|}{ State Point ${ }^{a}$} & \multicolumn{2}{|r|}{ State Point } \\
\hline 56.5 . & $\begin{array}{l}\mathrm{W}=0.8011 \times 10^{6} \\
\mathrm{P}=210.6 \mathrm{psia} \\
\mathrm{T}=105^{\circ} \mathrm{F}\end{array}$ & $1 \mathrm{bm} / \mathrm{hr}$ & 64. & $\begin{array}{l}\mathrm{W}=0.4738 \times 10^{6} 1 \mathrm{bm} / \mathrm{hr} \\
\mathrm{P}=406 \dot{\mathrm{o}}^{5} \mathrm{psia} \\
\mathrm{T}=182^{\mathrm{F}} \mathrm{F}\end{array}$ \\
\hline 57 . & $\begin{array}{l}\mathrm{W}=0.8125 \times 10^{6} \\
\mathrm{P}=210.6 \mathrm{psia} \\
\mathrm{T}=105^{\circ} \mathrm{F}\end{array}$ & $1 \mathrm{bm} / \mathrm{hr}$ & 65. & $\begin{array}{l}\mathrm{W}=0.4738 \times 10^{6} \mathrm{1bm} / \mathrm{hr} \\
\mathrm{P}=406.5 \mathrm{psia} \\
\mathrm{T}=240 \mathrm{~F}\end{array}$ \\
\hline 58 . & $\begin{array}{l}\mathrm{W}=0.8125 \times 10^{6} \\
\mathrm{P}=210.6 \mathrm{psia} \\
\mathrm{T}=180_{\mathrm{F}}^{\mathrm{F}}\end{array}$ & $1 \mathrm{bm} / \mathrm{hr}$ & 71. & $\begin{aligned} \mathrm{W}_{\mathrm{C} 4} & =112001 \mathrm{bm} / \mathrm{hr} \\
\mathrm{W}_{\mathrm{STM}} & =501 \mathrm{bm} / \mathrm{hr} \\
\mathrm{W}_{\mathrm{N}_{2}} & =1201 \mathrm{bm} / \mathrm{hr} \\
\mathrm{W}_{\mathrm{O}_{2}} & =401 \mathrm{bm} / \mathrm{hr} \\
2 & =79.78 \mathrm{psia} \\
\mathrm{P} & =104^{\circ} \mathrm{F}\end{aligned}$ \\
\hline 59. & $\begin{array}{l}W=0.3387 \times 10^{6} \\
P=210.6 \mathrm{psia} \\
T=180_{\mathrm{F}}\end{array}$ & $1 \mathrm{bm} / \mathrm{hr}$ & 72 . & $\begin{array}{l}\mathrm{W}=11220 \quad 1 \mathrm{bm} / \mathrm{hr} \\
\mathrm{P}=432 \mathrm{psia} \\
\mathrm{T}=105_{\mathrm{F}}^{\mathrm{O}}\end{array}$ \\
\hline 60 . & $\begin{aligned} \mathrm{W}_{\mathrm{C} 4} & =0.3387 \times 10^{6} \\
\mathrm{~W}_{\mathrm{STM}} & =38881 \mathrm{bm} / \mathrm{hr} \\
\mathrm{P} & =210.6 \mathrm{psia} \\
\mathrm{T} & =180^{\circ} \mathrm{F}\end{aligned}$ & $1 \mathrm{bm} / \mathrm{hr}$ & 73. & $\begin{array}{l}\mathrm{W}=36 \mathrm{1bm} / \mathrm{hr} \\
\mathrm{P}=38 \mathrm{psia} \\
\mathrm{T}=60^{\circ} \mathrm{F}\end{array}$ \\
\hline 62 . & $\begin{aligned} \mathrm{W}_{\mathrm{C} 4} & =0.3387 \times 10^{6} \\
\mathrm{~W}_{\mathrm{H}_{2} \mathrm{O}} & =38881 \mathrm{bm} / \mathrm{hr} \\
\mathrm{P} & =79.78 \mathrm{psia} \\
\mathrm{T}^{\mathrm{b}} & =126^{\circ} \mathrm{F}\end{aligned}$ & $1 \mathrm{bm} / \mathrm{hr}$ & 75 . & $\begin{array}{l}\mathrm{W}=11410 \quad \mathrm{lbm} / \mathrm{hr} \\
\mathrm{P}=432 \mathrm{psia} \\
\mathrm{T}=248^{\circ} \mathrm{F}\end{array}$ \\
\hline 63. & $\begin{array}{l}\mathrm{W}=0.4738 \times 10^{6} \\
\mathrm{P}=210.6 \mathrm{psia} \\
\mathrm{T}=180^{\circ} \mathrm{F}\end{array}$ & $1 \mathrm{bm} / \mathrm{hr}$ & 77. & $\begin{aligned} \mathrm{W}_{\mathrm{C} 4} & =251 \mathrm{bm} / \mathrm{hr} \\
\mathrm{W}_{\mathrm{N}_{2}} & =1201 \mathrm{bm} / \mathrm{hr} \\
\mathrm{W}_{\mathrm{O}_{2}} & =401 \mathrm{bm} / \mathrm{hr} \\
\mathrm{W}_{\mathrm{STM}} & =11 \mathrm{bm} / \mathrm{hr} \\
\mathrm{P} & =128 \mathrm{psia} \\
\mathrm{T} & =105 \mathrm{~F}\end{aligned}$ \\
\hline
\end{tabular}


State Point ${ }^{a}$

79.

$$
\begin{aligned}
& \mathrm{W}=11410 \mathrm{1bm} / \mathrm{hr} \\
& \mathrm{P}=210.6 \mathrm{psia} \\
& \mathrm{T}=106^{\circ} \mathrm{F}
\end{aligned}
$$

81. $\mathrm{W}_{\mathrm{H}_{2} \mathrm{O}}=6.598 \times 10^{6} \mathrm{lbm} / \mathrm{hr}$

$$
\begin{aligned}
\mathrm{W}_{\mathrm{C} 4} & =51 \mathrm{bm} / \mathrm{hr} \\
\mathrm{P} & =86{ }^{\mathrm{psia}} \\
\mathrm{T} & =96 \mathrm{~F}
\end{aligned}
$$

82. $\quad \begin{aligned} & \mathrm{W}=6.598 \times 10^{6} \mathrm{1bm} / \mathrm{hr} \\ & \mathrm{P}=14.7 \mathrm{psia} \\ & \mathrm{T}=75_{\mathrm{F}}\end{aligned}$

83.

$$
\begin{aligned}
& \mathrm{W}=6.598 \times 10^{6} \mathrm{1bm} / \mathrm{hr} \\
& \mathrm{P}=79.78 \mathrm{psia} \\
& \mathrm{T}=95^{\circ} \mathrm{F}
\end{aligned}
$$

87.

$$
\begin{aligned}
& \mathrm{W}=6.598 \times 10^{6} 1 \mathrm{bm} / \mathrm{hr} \\
& \mathrm{P}=79.78 \mathrm{psia} \\
& \mathrm{T}=95^{\circ} \mathrm{F}
\end{aligned}
$$

State Point

89.

$$
\begin{aligned}
& W=6.598 \times 10^{6} \mathrm{lbm} / \mathrm{hr} \\
& \mathrm{P}=79.78 \text { psia } \\
& \mathrm{T}=75^{\circ} \mathrm{F}
\end{aligned}
$$

90.

$$
\begin{aligned}
& \mathrm{W}=6.598 \times 10^{6} \mathrm{lbm} / \mathrm{hr} \\
& \mathrm{P}=1.31 \mathrm{psia} \\
& \mathrm{T}=95^{\circ} \mathrm{F}
\end{aligned}
$$

91.

$$
\begin{aligned}
\mathrm{W}_{\mathrm{STM}} & =5641 \mathrm{bm} / \mathrm{hr} \\
\mathrm{W}_{\mathrm{N}_{2}} & =651 \mathrm{bm} / \mathrm{hr} \\
\mathrm{W}_{\mathrm{O}}^{2} & =361 \mathrm{bm} / \mathrm{hr} \\
\mathrm{W}_{2} & =9241 \mathrm{bm} / \mathrm{hr} \\
\mathrm{C}_{\mathrm{C}} & =1.31 \mathrm{psia} \\
\mathrm{P} & =96^{\circ} \mathrm{F}
\end{aligned}
$$

92.

$$
\begin{aligned}
\mathrm{W} & =1589 \mathrm{1bm} / \mathrm{hr} \\
\mathrm{P} & =79.78 \mathrm{psia} \\
\mathrm{T}^{\mathrm{b}} & =547^{\circ} \mathrm{F}
\end{aligned}
$$

93.

$$
\begin{aligned}
& \mathrm{W}=0.118 \times 10^{6} \mathrm{lbm} / \mathrm{hr} \\
& \mathrm{P}=14.7 \mathrm{psia} \\
& \mathrm{T}=60^{\circ} \mathrm{F}
\end{aligned}
$$

a. State point numbers correspond to locations shown on Figure 6 .

b. Temperatures correspond to isentropic process; actual temperatures are higher. 
POWER BALANCE FOR DIRECT-CONTACT, DUAL-BOILING ISOBUTANE CYCLE

\begin{tabular}{|c|c|c|}
\hline & $\begin{array}{l}\text { Power } \\
(M W)\end{array}$ & $\begin{array}{c}\text { Penalties Assignable } \\
\text { to Direct-Contact Cycle } \\
(\mathrm{MW})\end{array}$ \\
\hline $\begin{array}{l}\text { Turbines at } \mathrm{P}_{\mathrm{N}_{2}} \text { Condenser } \\
\quad=2.2 \text { psia; }\end{array}$ & $3.56+1.48=5.04$ & $\sim 0$ \\
\hline $\begin{array}{l}\text { Turbines at } \mathrm{P}_{\mathrm{N}_{2}} \text { Condenser } \\
\quad=0\end{array}$ & 5.126 & -- \\
\hline $\begin{array}{l}\text { Geo-fluid pressure-drop } \\
\text { expander }(\eta=0.8)\end{array}$ & 0.131 & -- \\
\hline $\begin{array}{l}\text { Postflash geo-f1uid } \\
\text { expander }(\eta=0.6)\end{array}$ & 0.111 & -- \\
\hline $\begin{array}{l}\text { Cooling-water flash expander } \\
\qquad(n=0.6)\end{array}$ & 0.271 & -- \\
\hline $\begin{array}{l}\text { Incremental power due to } \\
-5^{\circ} \mathrm{F} \text { change in boiler/ } \\
\text { heater pinch points }\end{array}$ & 0.293 & -- \\
\hline $\begin{array}{l}\text { Incremental power due to } \\
-4^{\circ} \mathrm{F} \text { change in } \\
\text { condenser pinch point }\end{array}$ & 0.204 & -- \\
\hline
\end{tabular}


TABLE IX (continued)

\begin{tabular}{|c|c|c|}
\hline & $\begin{array}{l}\text { Parasitic } \\
\text { Losses } \\
\text { (MW) }\end{array}$ & $\begin{array}{c}\text { Penalties Assignable } \\
\text { to Direct-Contact Cycle } \\
(\mathrm{MW})\end{array}$ \\
\hline Basic wet cooling tower & 0.510 & $\sim 0$ \\
\hline High-pressure feed pump & 0.216 & 0.019 \\
\hline Low-pressure feed pump & 0.215 & 0.007 \\
\hline Geo-fluid delivery pump & 0.380 & 0.357 \\
\hline Geo-fluid reinjection pump & 0.104 & 0.127 \\
\hline $\begin{array}{l}\text { Geo-fluid postflash vapor } \\
\text { pump }\end{array}$ & 0.007 & 0.007 \\
\hline Condenser-vent pump & 0.042 & 0.042 \\
\hline $\begin{array}{l}\text { Turbine loss due to } \\
\qquad \mathrm{P}_{\mathrm{N}_{2}} \text { Condenser }=2.2 \text { psia }\end{array}$ & 0.086 & 0.086 \\
\hline $\begin{array}{l}\text { Condenser pump increment due } \\
\text { to cooling-water flash }\end{array}$ & 0.077 & 0.077 \\
\hline Cooling-water boost pumps & 0.562 & 0.562 \\
\hline $\begin{array}{l}\text { Cooling-water flash vapor } \\
\text { pump }\end{array}$ & 0.115 & 0.115 \\
\hline
\end{tabular}




\section{TABLE X}

STATE POINTS FOR DIRECT-CONTACT, TRIPLE-BOILING, TRIPLE-CONDENSING ISOBUTANE CYCLE

State Point ${ }^{a}$

1.

$$
\begin{aligned}
\mathrm{W}_{\mathrm{N}_{2}} / \mathrm{w}_{1} & =52 \times 10^{-6} \\
\mathrm{~W}_{\mathrm{H}_{2} \mathrm{O}} / \mathrm{w}_{1} & =1.0000 \\
\mathrm{P} & =100 \mathrm{psia} \\
\mathrm{T} & =290 \mathrm{~F}
\end{aligned}
$$

$1.5 \quad \mathrm{~W}_{\mathrm{STM}} / \mathrm{W}_{1}=0.00121$$$
\mathrm{w}_{\mathrm{N}_{2}} / \mathrm{w}_{1}=0.00005
$$$$
P=56.67+1.52
$$$$
=58.2 \text { psia }
$$$$
\mathrm{T}=289^{\circ} \mathrm{F}
$$

1.6

$$
\begin{aligned}
& \mathrm{W}_{\mathrm{H}_{2} \mathrm{O}} / \mathrm{W}_{1}=0.9988 \\
& \mathrm{w}_{\mathrm{N}_{2}} / \mathrm{w}_{1}=1.67 \times 10^{-6} \\
& \begin{array}{l}
\mathrm{P}=58.2 \mathrm{psia} \\
\mathrm{T}=289^{\mathrm{O}} \mathrm{F}
\end{array}
\end{aligned}
$$

1.7

$$
\begin{aligned}
\mathrm{W}_{\mathrm{H}_{2} \mathrm{O}} / \mathrm{W}_{1} & =0.00106 \\
\mathrm{~W}_{\mathrm{N}_{2}} / \mathrm{W}_{1} & =11 \times 10^{-6} \\
\mathrm{P} & =58.2 \mathrm{psia} \\
\mathrm{T} & =278^{\circ} \mathrm{F}
\end{aligned}
$$

6.

5.

4.

$$
\begin{aligned}
\mathrm{W}_{4} / \mathrm{W}_{1} & =0.9945 \\
\mathrm{P} & =496.9 \mathrm{psia} \\
\mathrm{T} & =265^{\circ} \mathrm{F}
\end{aligned}
$$

\section{State Point}

3.

$$
\begin{aligned}
\mathrm{W}_{3} / \mathrm{W}_{1} & =0.9999 \\
\mathrm{P} & =496.9 \text { psia } \\
\mathrm{T} & =290{ }_{\mathrm{F}}
\end{aligned}
$$




\begin{tabular}{|c|c|c|c|}
\hline & State Point ${ }^{\mathrm{a}}$ & & State Point \\
\hline 10 & $\begin{aligned} \mathrm{W}_{10} / \mathrm{W}_{1} & =0.9900 \\
\mathrm{P} & =145.7 \mathrm{psia} \\
\mathrm{T} & =155^{\mathrm{o}} \mathrm{l}\end{aligned}$ & 19. & $\begin{aligned} \mathrm{W}_{19} / \mathrm{W}_{1} & =8.31 \\
\mathrm{~T} & =75^{\circ} \mathrm{F}\end{aligned}$ \\
\hline 11. & $\begin{aligned} \mathrm{W}_{\mathrm{H}_{2} \mathrm{O}} / \mathrm{W}_{1} & =0.9900 \\
\mathrm{~W}_{\mathrm{WF}} / \mathrm{W}_{1} & =0.000144 \\
\mathrm{P} & =145.7 \mathrm{psia} \\
\mathrm{T} & =128^{\circ} \mathrm{F}\end{aligned}$ & 20 & $\begin{aligned} \mathrm{W}_{20} / \mathrm{W}_{1} & =8.31 \\
\mathrm{~T} & =82^{\circ} \mathrm{F}\end{aligned}$ \\
\hline 12. & $\begin{aligned} \mathrm{W}_{\mathrm{H}_{2} \mathrm{O}} / \mathrm{W}_{1} & =0.9900 \\
\mathrm{~W}_{\mathrm{WF}} / \mathrm{W}_{1} & =5 \times 10^{-6} \\
\mathrm{P} & =7.4 \mathrm{opsia} \\
\mathrm{T} & =128 \mathrm{~F}\end{aligned}$ & 21 & $\begin{aligned} \mathrm{W}_{21} / \mathrm{W}_{1} & =6.19 \\
\mathrm{~T} & =90^{\circ} \mathrm{F}\end{aligned}$ \\
\hline 13. & $\begin{aligned} \mathrm{W}_{13} / \mathrm{W}_{1} & =0.9900 \\
\mathrm{P} & =100{ }^{\mathrm{psia}} \\
\mathrm{T} & =128 \mathrm{~F}\end{aligned}$ & 22 . & $\begin{aligned} \mathrm{W}_{22} / \mathrm{W}_{1} & =6.72 \\
\mathrm{~T} & =92.5^{\circ} \mathrm{F}\end{aligned}$ \\
\hline 14. & $\begin{array}{l}\text { Essentially the same as } \\
13 \text {. }\end{array}$ & 31 & $\begin{aligned} \mathrm{W}_{31} / \mathrm{W}_{1} & =30 \times 10^{-6} \\
\mathrm{P} & =78.6 \mathrm{psia} \\
\mathrm{T} & =104^{\mathrm{o}} \mathrm{F}\end{aligned}$ \\
\hline 15 . & $\begin{aligned} \mathrm{W}_{\mathrm{WF}} / \mathrm{W}_{1} & =139 \times 10^{-6} \\
\mathrm{~W}_{\mathrm{STM}} / \mathrm{W}_{1} & =21 \times 10^{-6} \\
\mathrm{P} & =7.4 \mathrm{psia} \\
\mathrm{T} & =128 \mathrm{~F}\end{aligned}$ & 51. & $\begin{aligned} \mathrm{W}_{\mathrm{WF}} / \mathrm{W}_{1} & =0.4402 \\
\mathrm{~W}_{\mathrm{STM}} / \mathrm{W}_{1} & =0.00528 \\
\mathrm{P} & =496 \dot{\circ}_{\mathrm{F}}^{9} \mathrm{psia} \\
\mathrm{T} & =260^{\circ}\end{aligned}$ \\
\hline 16. & $\begin{aligned} \mathrm{W}_{16} / \mathrm{W}_{1} & =160 \times 10^{-6} \\
\mathrm{P} & =78.6 \mathrm{psia} \\
\mathrm{T}^{\mathrm{b}} & =274^{\circ} \mathrm{F}\end{aligned}$ & 52 . & $\begin{aligned} \mathrm{W}_{52} / \mathrm{W}_{1} & =0.4455 \\
\mathrm{P} & =64.7 \mathrm{psia} \\
\mathrm{T}^{\mathrm{b}} & =120^{\circ} \mathrm{F}\end{aligned}$ \\
\hline 17. & $\begin{aligned} \mathrm{W}_{17} / \mathrm{W}_{1} & =8.31 \\
\mathrm{~T} & =75^{0} \mathrm{~F}\end{aligned}$ & 53. & $\begin{aligned} \mathrm{W}_{53} / \mathrm{W}_{1} & =0.4455 \\
\mathrm{P} & =64.7 \text { psia } \\
\mathrm{T} & =91^{\circ} \mathrm{F}\end{aligned}$ \\
\hline 18. & $\begin{aligned} \mathrm{W}_{18} / \mathrm{W}_{1} & =8.31 \\
\mathrm{~T} & =75^{\circ} \mathrm{F}\end{aligned}$ & 54. & $\begin{aligned} \mathrm{W}_{54} / \mathrm{W}_{1} & =0.4453 \\
\mathrm{P} & =64 \dot{\mathrm{o}}_{\mathrm{F}}^{7} \mathrm{psia} \\
\mathrm{T} & =91\end{aligned}$ \\
\hline
\end{tabular}


State Point ${ }^{a}$

55.

$$
\begin{aligned}
\mathrm{W}_{55} / \mathrm{W}_{1} & =0.4453 \\
\mathrm{P} & =73.0 \text { psia } \\
\mathrm{T} & =99^{\mathrm{O}}
\end{aligned}
$$

56.

$$
\begin{aligned}
\mathrm{W}_{56} / \mathrm{W}_{1} & =0.9424 \\
\mathrm{P} & =145.7 \text { psia }
\end{aligned}
$$

57. Essentially the same as 56.

58.

$$
\begin{aligned}
\mathrm{W}_{58} / \mathrm{W}_{1} & =0.9424 \\
\mathrm{P} & =145.7 \text { psia } \\
\mathrm{T} & =150^{\circ} \mathrm{F}
\end{aligned}
$$

59.

$$
\begin{aligned}
\mathrm{W}_{59} / \mathrm{W}_{1} & =0.2269 \\
\mathrm{P} & =145.7 \text { psia } \\
\mathrm{T} & =150^{\circ} \mathrm{F}
\end{aligned}
$$

61.

$$
\begin{aligned}
\mathrm{W}_{\mathrm{WF}} / \mathrm{W}_{1} & =0.2753 \\
\mathrm{~W}_{\mathrm{STM}} / \mathrm{W}_{1} & =0.0030 \\
\mathrm{P} & =296.0 \mathrm{psia} \\
\mathrm{T} & =210^{\circ} \mathrm{F}
\end{aligned}
$$

62.

$$
\begin{aligned}
\mathrm{W}_{62} / \mathrm{w}_{1} & =0.2783 \\
\mathrm{P} & =73.0 \mathrm{psia} \\
\mathrm{T}^{\mathrm{b}} & =123^{\circ} \mathrm{F}
\end{aligned}
$$

63.

$$
\begin{aligned}
\mathrm{W}_{63} / \mathrm{W}_{1} & =0.7155 \\
\mathrm{P} & =145.7 \text { psia } \\
\mathrm{T} & =150^{\circ} \mathrm{F}
\end{aligned}
$$

64.

$$
\begin{aligned}
\mathrm{W}_{64} / \mathrm{W}_{1} & =0.7155 \\
\mathrm{P} & =296.0 \text { psia } \\
\mathrm{T} & =151 \mathrm{~F}
\end{aligned}
$$

65.

$$
\begin{aligned}
\mathrm{W}_{65} / \mathrm{W}_{1} & =0.7155 \\
\mathrm{P} & =296.0 \text { psia } \\
\mathrm{T} & =210_{\mathrm{F}}^{\circ}
\end{aligned}
$$

State Point

66.

$$
\begin{aligned}
\mathrm{W}_{66} / \mathrm{W}_{1} & =0.2753 \\
\mathrm{P} & =296.0 \text { psia } \\
\mathrm{T} & =210^{\circ} \mathrm{F}
\end{aligned}
$$

67.

$$
\begin{aligned}
\mathrm{W}_{67} / \mathrm{W}_{1} & =0.4402 \\
\mathrm{P} & =296.0 \mathrm{psia} \\
\mathrm{T} & =210^{\circ} \mathrm{F}
\end{aligned}
$$

68.

$$
\begin{aligned}
\mathrm{W}_{68} / \mathrm{W}_{1} & =0.4402 \\
\mathrm{P} & =496.9 \mathrm{psia} \\
\mathrm{T} & =21 \mathrm{o}_{\mathrm{F}}
\end{aligned}
$$

69.

$$
\begin{aligned}
\mathrm{W}_{69} / \mathrm{W}_{1} & =0.4402 \\
\mathrm{P} & =496.9 \mathrm{psia} \\
\mathrm{T} & =260{ }^{\circ} \mathrm{F}
\end{aligned}
$$

69.5

$$
\begin{aligned}
\mathrm{W}_{69.5} / \mathrm{W}_{1} & =0.0204 \\
\mathrm{P} & =496.9 \text { psia } \\
\mathrm{T} & =260_{\mathrm{F}}^{\circ}
\end{aligned}
$$

69.6

$$
\begin{aligned}
\mathrm{W}_{69.6} / \mathrm{W}_{1}= & 0.0204 \\
\mathrm{P}= & 496.9 \text { psia } \\
\mathrm{T}= & 268^{\circ} \mathrm{F} \\
& (\text { Sat. Vapor })
\end{aligned}
$$

69.7

$$
\begin{aligned}
\mathrm{W}_{69.7} / \mathrm{W}_{1} & =0.4198 \\
\mathrm{P} & =496.9 \text { psia } \\
\mathrm{T} & =260{ }^{\circ} \mathrm{F}
\end{aligned}
$$

71.

$$
\begin{aligned}
\mathrm{W}_{\mathrm{WF}} / \mathrm{W}_{1} & =0.2269 \\
\mathrm{~W}_{\mathrm{STM}} / \mathrm{W}_{1} & =0.0015 \\
\mathrm{P} & =145.7 \mathrm{psia} \\
\mathrm{T} & =150^{\circ} \mathrm{F}
\end{aligned}
$$

72.

$$
\begin{aligned}
\mathrm{W}_{72} / \mathrm{W}_{1} & =0.2284 \\
\mathrm{P} & =78.6 \text { psia } \\
\mathrm{T}^{\mathrm{b}} & =126^{\circ} \mathrm{F}
\end{aligned}
$$

73.

$$
\begin{aligned}
\mathrm{W}_{73} / \mathrm{W}_{1} & =0.7236 \\
\mathrm{P} & =73.0 \text { psia } \\
\mathrm{T} & =99^{\circ} \mathrm{F}
\end{aligned}
$$


State Point

74. Same as 73, but condensation complete.

$$
\begin{aligned}
\mathrm{W}_{75} / \mathrm{W}_{1} & =0.7236 \\
\mathrm{P} & =78.6 \text { psia } \\
\mathrm{T} & =99 \mathrm{o}_{\mathrm{F}}
\end{aligned}
$$

76.

$$
\begin{aligned}
\mathrm{w}_{76} / \mathrm{W}_{1} & =0.9522 \\
\mathrm{P} & =78.6 \mathrm{psia} \\
\mathrm{T} & =104^{\circ} \mathrm{F}
\end{aligned}
$$

77. Same as 76 , but condensation complete.

83.

81.

$$
\begin{aligned}
\mathrm{W}_{\mathrm{WF}} / \mathrm{W}_{1} & =218 \times 10^{-6} \\
\mathrm{~W}_{\mathrm{STM}} / \mathrm{W}_{1} & =0.8 \times 10^{-6} \\
\mathrm{~W}_{\mathrm{N}_{2}} / \mathrm{W}_{1} & =1.7 \times 10^{-6} \\
\mathrm{P} & =64.7 \mathrm{psia} \\
\mathrm{T} & =91{ }_{\mathrm{F}}^{\circ}
\end{aligned}
$$

82.

Essentially the same as 81 .

$$
\begin{aligned}
\mathrm{W}_{83} / \mathrm{W}_{1} & =220 \times 10^{-6} \\
\mathrm{P} & =65.0 \mathrm{psia} \\
\mathrm{T} & =91 \mathrm{O}_{\mathrm{F}}
\end{aligned}
$$

84.

$$
\begin{aligned}
\mathrm{W}_{84 / \mathrm{W}_{1}} & =220 \times 10^{-6} \\
\mathrm{P} & =65 \dot{\mathrm{o}}^{0} \text { psia } \\
\mathrm{T} & =84^{\circ} \mathrm{F}
\end{aligned}
$$

85.

$$
\begin{aligned}
\mathrm{W}_{\mathrm{WF}} / \mathrm{W}_{1} & =193 \times 10^{-6} \\
\mathrm{~W}_{\mathrm{STM}} / \mathrm{W}_{1} & =0.7 \times 10^{-6} \\
\mathrm{P} & =65.0 \mathrm{psia} \\
\mathrm{T} & =84^{\circ} \mathrm{F}
\end{aligned}
$$$$
P=78.6 \text { psia }
$$

79. Essentially the same as 78 .

87.

$$
\begin{aligned}
\mathrm{W}_{\mathrm{WF}} / \mathrm{w}_{1} & =25 \times 10^{-6} \\
\mathrm{~W}_{\mathrm{STM}} / \mathrm{w}_{1} & =0.1 \times 10^{-6} \\
\mathrm{~W}_{\mathrm{N}_{2}} / \mathrm{W}_{1} & =1.7 \times 10^{-6} \\
\mathrm{P} & =65.0 \mathrm{psia} \\
\mathrm{T} & =84^{\circ} \mathrm{F}
\end{aligned}
$$

80.

$$
\begin{aligned}
\mathrm{W}_{80} / \mathrm{W}_{1} & =0.0098 \\
\mathrm{P} & =78.6 \mathrm{psia} \\
\mathrm{T} & =104^{\circ} \mathrm{F}
\end{aligned}
$$

a. State point numbers correspond to locations shown on Figure 5.

b. Temperatures correspond to isentropic process; actual temperatures are higher. 
TABLE XI

POWER BALANCE FOR TRIPLE-BOILING, TRIPLE-CONDENSING, DIRECT-CONTACT ISOBUTANE CYCLE

Direct-Contact Boiler Conditions $\left(5^{\circ} \mathrm{F}\right.$ pinch points): $260^{\circ} \mathrm{F}, 210^{\circ} \mathrm{F}, 150^{\circ} \mathrm{F} ; 497,296,146 \mathrm{psia}$

She11-and-Tube Condensing Conditions ( $9^{\circ} \mathrm{F}$ pinch points):

$91^{\circ} \mathrm{F}, 99^{\circ} \mathrm{F}, 104^{\circ} \mathrm{F} ; 64.7,73.0,78.6 \mathrm{psia}$

(for high-, medium-, and low-pressure-turbine condensers)

Turbine Power: 3.860.1.742, 0.623 (high-, medium-, low-pressure turbines)

Total $=6.225$ watt $-\mathrm{hr} / 1 \mathrm{bm}$ geo-fluid

Auxiliary Expander Power: $0.004,0.024,0.148,0.107,0.073$

Total $=0.355$ watt $-\mathrm{hr} / 1 \mathrm{bm}$ geo-fluid

Working-Fluid Feed Pump Power:

0.606 watt-hr/ $1 \mathrm{bm}$ geo-fluid

Geo-Fluid Delivery Pump Power ( 58.2 to 497 psia):

0.515 watt $-\mathrm{hr} / 1 \mathrm{bm}$ geo-fluid

Geo-Fluid Reinjection Pump Power ( 7.4 to 100 psia):

0.101 watt-hr/lbm geo-fluid

Geo-Fluid Postflash Vapor Compression Power:

0.004 watt-hr/1bm geo-fluid

Condenser-Vent Compression Power:

$0 \quad(<0.0001-$ watt $\mathrm{hr} / 1 \mathrm{bm}$ geo-fluid)

Wet Cooling Tower Power:

0.573 watt $-\mathrm{hr} / \mathrm{lbm}$ geo-fluid

Net Plant Power:

4.781 watt-hr/1bm geo-fluid 
TABLE XII

STATE POINTS FOR TRIPLE-BOILING, TRIPLE-CONDENSING, SHELL-AND-TUBE ISOBUTANE CYCLE

Geo-Fluid In

High-Pressure Boiler

$$
\mathrm{T}=290^{\circ} \mathrm{F}
$$

$\mathrm{T}=270^{\circ} \mathrm{F}$

High-Pressure Heater
$\mathrm{T}=249^{\circ} \mathrm{F}$

$$
\mathrm{T}=270^{\circ} \mathrm{F}
$$

Geo-Fluid Out

Working Fluid In
$\mathrm{T}=260^{\circ} \mathrm{F}$

$\mathrm{T}=260^{\circ} \mathrm{F}$

$\mathrm{P}=461.4 \mathrm{psia}$

$\mathrm{W} / \mathrm{W}_{\mathrm{Geo}-\mathrm{fluid}}=0.423$ 
Geo-Fluid In Geo-Fluid Out Working Fluid In

Working Fluid Out

Medium-Pressure Heater

$$
\mathrm{T}=220^{\circ} \mathrm{F}
$$

$\mathrm{T}=193^{\circ} \mathrm{F}$

$\mathrm{T}=160^{\circ} \mathrm{F}$

$\mathrm{T}=210^{\circ} \mathrm{F}$

$$
\mathrm{P}=281.9 \mathrm{psia}
$$

$$
\mathrm{W} / \mathrm{W}_{\text {Geo-fluid }}=0.743
$$

Medium-Pressure Turbine

$$
\begin{array}{cc}
\mathrm{T}=210^{\circ} \mathrm{F} & \mathrm{T}^{\mathrm{a}}=119^{\circ} \mathrm{F} \\
\mathrm{P}=281.9 \text { psia } & \mathrm{P}=71.8 \text { psia } \\
\mathrm{W} / \mathrm{W}_{\text {Geo-fluid }}=0.320
\end{array}
$$

Cooling Water In Cooling Water Out Working Fluid In Working Fluid Out Medium-Pressure Turbine Condenser

$$
\begin{array}{rr}
\mathrm{T}=84^{\circ} \mathrm{F}=90.8^{\circ} \mathrm{F} & \mathrm{T}=99.8^{\circ} \mathrm{F} \\
\mathrm{P}=71.8 \text { psia } \\
\mathrm{W} / \mathrm{W}_{\text {Geo-fluid }}=7.16 & \mathrm{~W} / \mathrm{W}_{\mathrm{Geo}-\mathrm{fluid}}=0.320
\end{array}
$$

Geo-Fluid In Geo-Fluid Out

Working Fluid In Working Fluid Out
$\mathrm{T}=193^{\circ} \mathrm{F}$

$\mathrm{T}=170^{\circ} \mathrm{F}$

$\mathrm{T}=137^{\circ} \mathrm{F}$

$\mathrm{T}=170^{\circ} \mathrm{F}$
$\mathrm{T}=160^{\circ} \mathrm{F}$

$\mathrm{T}=160^{\circ} \mathrm{F}$

$\mathrm{P}=160.6$ psia

$\mathrm{W} / \mathrm{W}_{\mathrm{Geo}} \mathrm{f} 1 \mathrm{uid}=0.198$

Low-Pressure Heater

$$
\begin{gathered}
\mathrm{T}=104^{\circ} \mathrm{F} \quad \mathrm{T}=160^{\circ} \mathrm{F} \\
\mathrm{P}=160.6 \text { psia } \\
\mathrm{W} / \mathrm{W}_{\text {Geo-fluid }}=0.940
\end{gathered}
$$




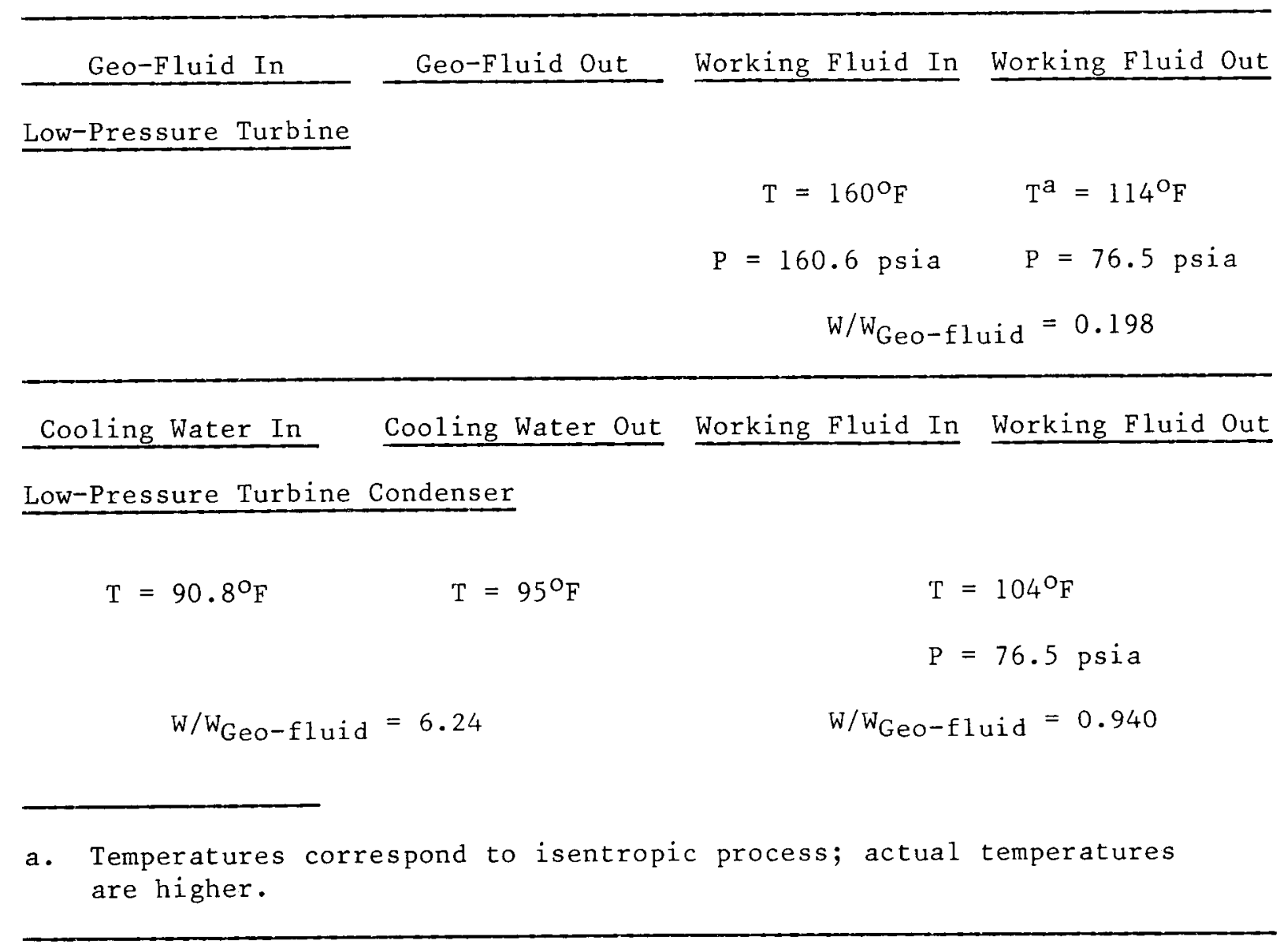


$\underline{\text { TABLE XIII }}$

POWER BALANCE FOR TRIPLE-BOILING, TRIPLE-CONDENSING, SHELL-AND-TUBE ISOBUTANE CYCLE

Boiler Conditions $\left(10^{\circ} \mathrm{F}\right.$ pinch points):

$260^{\circ} \mathrm{F}, 210^{\circ} \mathrm{F}, 160^{\circ} \mathrm{F} ; 461,282,72$ psia

Condensing Conditions $\left(9^{\circ} \mathrm{F}\right.$ pinch points):

$93^{\circ} \mathrm{F}, 99.8^{\circ} \mathrm{F}, 104^{\circ} \mathrm{F} ; 65,72,76.5$ psia

(for high-, medium-, and low-pressure turbine condensers)

Turbine Power: $3.384,1.890,0.640$ (high-, medium-, low-pressure turbines)

Total $=5.914$ watt $-\mathrm{hr} / 1 \mathrm{bm}$ geo-fluid

Working-Fluid Feed Pump Power:

0.570 watt-hr/ $1 \mathrm{bm}$ geo-fluid

Wet Cooling Tower Power:

0.520 watt-hr/ $1 \mathrm{bm}$ geo-fluid

Net Plant Power:

4.824 watt-hr/1bm geo-fluid 


\section{TABLE XIV}

EFFECT OF BOILER TEMPERATURES ON CYCLE PERFORMANCE

FOR AN ISOBUTANE SHELL-AND-TUBE CYCLE

(Power Values in Watt-hr/1bm Geo-Fluid)

(Flows Divided by Geo-Fluid Flow)

\begin{tabular}{lcc}
\hline & \multicolumn{1}{c}{ Boiler Temperatures } \\
\cline { 2 - 2 } & $260,210,150^{\circ} \mathrm{F}$ & $250,200,150^{\circ} \mathrm{F}$ \\
High-pressure turbine power & 3.043 & 3.487 \\
Medium-pressure turbine power & 1.784 & 1.449 \\
Low-pressure turbine power & 0.621 & 0.497 \\
High-pressure turbine flow & 0.423 & 0.500 \\
Medium-pressure turbine flow & 0.320 & 0.282 \\
Low-pressure turbine flow & 0.234 & 0.188 \\
Working-fluid pump power & 0.552 & 0.526 \\
Gross plant power & 5.448 & 5.433 \\
Gross power - pump power $\approx$ net power & 4.896 & 4.907 \\
\hline
\end{tabular}


Internal Distribution

$$
\begin{aligned}
& 1 \text { - } \text { Chicago Patent Group - DOE } \\
& 9800 \text { South Cass } \\
& \text { Argonne, IL } 60439 \\
& 1 \text { - } \text { R. L. Blackledge } \\
& \text { Idaho Operations Office - DOE } \\
& \text { Idaho Falls, ID } 83401 \\
& 1 \text { - } \text { H. P. Pearson } \\
& \text { Information Processing - EG\&G } \\
& 6 \text { - } \text { INEL Technical Library } \\
& 55 \text { - Author }
\end{aligned}
$$

External Distribution

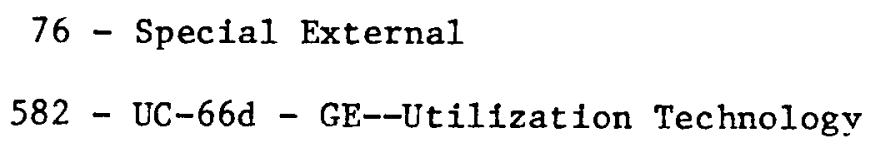

Total Copies Printed: 722 